(c) 2006 International Press

Adv. Theor. Math. Phys. 10 (2006) 759-851

\title{
Two-dimensional twisted sigma models and the theory of chiral differential operators
}

\author{
Meng-Chwan Tan \\ Department of Physics, National University of Singapore, \\ S(119260), Singapore \\ g0306155@nus.edu.sg
}

\begin{abstract}
In this paper, we study the perturbative aspects of a twisted version of the two-dimensional $(0,2)$ heterotic sigma model on a holomorphic gauge bundle $\mathcal{E}$ over a complex, hermitian manifold $X$. We show that the model can be naturally described in terms of the mathematical theory of "Chiral Differential Operators". In particular, the physical anomalies of the sigma model can be reinterpreted in terms of an obstruction to a global definition of the associated sheaf of vertex superalgebras derived from the free conformal field theory describing the model locally on $X$. One can also obtain a novel understanding of the sigma model 1-loop beta-function solely in terms of holomorphic data. At the $(2,2)$ locus, where the obstruction vanishes for any smooth manifold $X$, we obtain a purely mathematical description of the half-twisted variant of the topological A-model and (if $c_{1}(X)=0$ ) its elliptic genus. By studying
\end{abstract}

e-print archive: http://lanl.arXiv.org/abs/0604179v3 
the half-twisted $(2,2)$ model on $X=\mathbb{C P}^{1}$, one can show that a subset of the infinite-dimensional space of physical operators generates an underlying superaffine Lie algebra. Furthermore, on a non-Kähler, parallelized, group manifold with torsion, we uncover a direct relationship between the modulus of the corresponding sheaves of chiral de Rham complex and the level of the underlying WZW theory.

\section{Introduction}

The mathematical theory of "Chiral Differential Operators" (CDOs) is a fairly well-developed subject that aims to provide a rigorous mathematical construction of conformal fields theories, possibly associated with sigma models in two-dimensions, without resorting to mathematically non-rigorous methods such as the path integral. It was first introduced and studied in a series of seminal papers by Malikov and coworkers [1-5] and in [6] by Beilinson and Drinfeld, whereby a more algebraic approach to this construction was taken in the latter. These developments have found interesting applications in various fields of geometry and representation theory such as mirror symmetry [7] and the study of elliptic genera [8-10], just to name a few. However, the explicit interpretation of the theory of CDOs, in terms of the physical models it is supposed to describe, has been somewhat unclear, that is until recently.

In the pioneering papers of Kapustin [11] and Witten [12], initial steps were taken to provide a physical interpretation of some of the mathematical results in the general theory of CDOs. In [11], it was argued that on a Calabi-Yau manifold $X$, the mathematical theory of a CDO, known as the chiral de Rham (CDR) complex can be identified with the infinite-volume limit of a half-twisted variant of the topological A-model. And in [12], the perturbative limit of a half-twisted $(0,2)$ sigma model with right-moving fermions was studied, where its interpretation in terms of the theory of a $\mathrm{CDO}$ that is a purely bosonic version of the CDR was elucidated. And even more recently, an explicit computation (on $\mathbb{P}^{1}$ ) was carried out by Frenkel et al. in [13] to verify mathematically, the identification of the CDR as the half-twisted sigma model in perturbation theory.

In this paper, we will consider a generalization of the model considered in [12] to include left-moving worldsheet fermions valued in a holomorphic gauge bundle over the target space. To this end, we will be studying the perturbative aspects of a twisted version of the two-dimensional $(0,2)$ heterotic sigma model. Our main goal is to seek a physical interpretation of the mathematical theory of a general class of CDOs, constructed from generic 
vertex superalgebras, by Malikov and coworkers in $[3,5]$. In turn, we hope to obtain some novel insights into the physics via a reinterpretation of some established mathematical results. Additional motivation for this work also come from the fact that this generalization is important in the heterotic string theory. In fact, other various aspects of similar models have been extensively studied in the physics literature. Of particular physical importance would be the results obtained by Katz and Sharpe in [15], which suggest that under certain conditions, the correlation functions of physical operators in the model considered can be related to the Yukawa couplings in heterotic string compactifications. Various twisted heterotic sigma models were also used in [14] to ascertain the criteria for conformal invariance in $(0,2)$ models. Last but not the least, the existence of a topological heterotic ring of ground operators (which reduces to the $(a, c)$ ring of an untwisted $(2,2)$ model at the $(2,2)$ locus) in the conformal and "massive" limits of an isomorphic model was also investigated in [16]. This presents new possibilities for the application of physical insights in mathematics and vice-versa. To this end, we shall generalize Witten's approach in [12].

\subsection{A brief summary and plan of the paper}

A brief summary and plan of the paper is as follows. First, in Section 2, we will review the two-dimensional heterotic sigma model with $(0,2)$ supersymmetry on a rank- $r$ holomorphic gauge bundle $\mathcal{E}$ over a Kähler manifold $X$. We will then introduce a twisted variant of the model, obtained via a redefinition of the spins of the relevant worldsheet fields.

Next, in Section 3, we will focus on the space of physical operators of this twisted sigma model. In particular, we will study the properties of the chiral algebra furnished by these operators. In addition, we will show how the moduli of the chiral algebra arise when we include a non-Kähler deformation of $X$.

In Section 4, we will discuss, from a purely physical perspective, the anomalies of this specific model. The main aim in doing so is to prepare for the observations and results that we will make and find in the next section.

In Section 5, we will introduce the notion of a sheaf of perturbative observables. An alternative description of the chiral algebra of physical operators in terms of the elements of a Cech cohomology group will also be presented. Thereafter, we will show that the twisted model on a local patch of the target space can be described in terms of a free $b c-\beta \gamma$ system, where in order to give a complete description of the model on the entire target space, it will first 
be necessary to study its local symmetries. Using these local symmetries, one can then glue together the free conformal field theories (each defined on a local patch of the target space by the free $b c-\beta \gamma$ system) to obtain a globally defined sheaf of CDOs or vertex superalgebras which span a subset of the chiral algebra of the model. It is at this juncture that one observes the mathematical obstruction to a global definition of the sheaf (and hence the existence of the underlying theory) to be the physical anomaly of the model itself. Via an example, we will be able to obtain a novel understanding of the non-zero 1-loop beta-function of the twisted heterotic sigma model solely in terms of holomorphic data.

In Section 6, we will study the twisted model at the $(2,2)$ locus where $\mathcal{E}=T X$, for which the obstruction to a global definition of the sheaf of vertex superalgebras vanishes for any smooth manifold $X$. In doing so, we obtain a purely mathematical description of the half-twisted variant of Witten's topological A-model [17] in terms of a theory of a class of conformal vertex superalgebras called the CDR, which for a target space with vanishing first Chern class such as a Calabi-Yau manifold, acquires the structure of a topological vertex algebra. Our results therefore serve as an alternative verification and generalization of Kapustin's findings in [11] and Frenkel and Losev's computation in [13]. Using the CFT state-operator correspondence in the Calabi-Yau case, one can make contact with the mathematical definition of the elliptic genus introduced in [8-10] solely via physical considerations.

In Section 7, we will analyse, as examples, sheaves of CDR that describe the physics of the half-twisted $(2,2)$ model on two different smooth manifolds. The main aim is to illustrate the rather abstract discussion in the preceding sections. By studying the sheaves of $\mathrm{CDR}$ on $\mathbb{C P}^{1}$, we find that a subset of the infinite-dimensional space of physical operators furnishes an underlying superaffine Lie algebra. As in Section 5, we will be able to obtain a novel understanding of the non-zero 1-loop beta-function of the half-twisted sigma model solely in terms of holomorphic data. Furthermore, for the half-twisted $(2,2)$ model on a non-Kähler, parallelized, smooth manifold with torsion such as $S^{3} \times S^{1}$, a study of the corresponding sheaf of CDR reveals a direct relationship between the modulus of sheaves and the level of the underlying $S U(2)$ WZW theory.

\subsection{Beyond perturbation theory}

As pointed out in [12], instanton effects can change the picture radically, triggering a spontaneous breaking of supersymmetry, hence making the chiral 
algebra trivial as the elliptic genus vanishes. Thus, out of the perturbation theory, the sigma model may no longer be described by the theory of CDOs. This non-perturbative consideration is beyond the scope of the present paper. However, we do hope to address it in a future publication.

\section{A twisted heterotic sigma model}

\subsection{The heterotic sigma model with $(0,2)$ supersymmetry}

To begin, let us first recall the two-dimensional heterotic non-linear sigma model with $(0,2)$ supersymmetry on a rank- $r$ holomorphic gauge bundle $\mathcal{E}$ over a Kähler manifold $X$. It governs maps $\Phi: \Sigma \rightarrow X$, with $\Sigma$ being the worldsheet Riemann surface. By picking local coordinates $z, \bar{z}$ on $\Sigma$, and $\phi^{i}$, $\phi^{\bar{i}}$ on $X$, the map $\Phi$ can then be described locally via the functions $\phi^{i}(z, \bar{z})$ and $\phi^{\bar{i}}(z, \bar{z})$. Let $K$ and $\bar{K}$ be the canonical and anti-canonical bundles of $\Sigma$ (the bundles of one-forms of types $(1,0)$ and $(0,1)$, respectively), whereby the spinor bundles of $\Sigma$ with opposite chiralities are given by $K^{1 / 2}$ and $\bar{K}^{1 / 2}$. Let $T X$ and $\overline{T X}$ be the holomorphic and anti-holomorphic tangent bundle of $X$. The left-moving fermi fields of the model consist of $\lambda^{a}$ and $\lambda_{a}$, which are smooth sections of the bundles $K^{1 / 2} \otimes \Phi^{*} \mathcal{E}$ and $K^{1 / 2} \otimes \Phi^{*} \mathcal{E}^{*}$, respectively. On the other hand, the right-moving fermi fields consist of $\psi^{i}$ and $\psi^{\bar{i}}$, which are smooth sections of the bundles $\bar{K}^{1 / 2} \otimes \Phi^{*} T X$ and $\bar{K}^{1 / 2} \otimes \Phi^{*} \overline{T X}$, respectively. Here, $\psi^{i}$ and $\psi^{\bar{i}}$ are superpartners of the scalar fields $\phi^{i}$ and $\phi^{\bar{i}}$, while $\lambda^{a}$ and $\lambda_{a}$ are superpartners to a set of auxiliary scalar fields $l^{a}$ and $l_{a}$, which are in turn smooth sections of the bundles $K^{1 / 2} \otimes \bar{K}^{1 / 2} \otimes \Phi^{*} \mathcal{E}$ and $K^{1 / 2} \otimes \bar{K}^{1 / 2} \otimes \Phi^{*} \mathcal{E}^{*}$, respectively. Let $g$ be the hermitian metric on $X$. The action is then given by

$$
\begin{gathered}
S=\int_{\Sigma}\left|d^{2} z\right|\left(\frac{1}{2} g_{i \bar{j}}\left(\partial_{z} \phi^{i} \partial_{\bar{z}} \phi^{\bar{j}}+\partial_{\bar{z}} \phi^{i} \partial_{z} \phi^{\bar{j}}\right)+g_{i \bar{j}} \psi^{i} D_{z} \psi^{\bar{j}}+\lambda_{a} D_{\bar{z}} \lambda^{a}\right. \\
\left.+F_{b i \bar{j}}^{a}(\phi) \lambda_{a} \lambda^{b} \psi^{i} \psi^{\bar{j}}-l_{a} l^{a}\right),
\end{gathered}
$$

whereby $i, \bar{i}=1, \ldots, n=\operatorname{dim}_{\mathbb{C}} X, a=1, \ldots, r,{ }^{1}\left|d^{2} z\right|=i d z \wedge d \bar{z}$, and $F^{a}{ }_{b i \bar{j}}$ $(\phi)=A_{b i, \bar{j}}^{a}(\phi)$ is the curvature two-form of the holomorphic gauge bundle $\mathcal{E}$ with connection $A$. In addition, $D_{z}$ is the $\partial$ operator on $\bar{K}^{1 / 2} \otimes \phi^{*} \overline{T X}$

\footnotetext{
${ }^{1}$ As we will be studying the sigma model in the peturbative limit, worldsheet instantons are absent, and one considers only (degree zero) constant maps $\Phi$, such that $\int_{\Sigma} \Phi^{*} c_{1}(\mathcal{E})=$ 0 . Since the selection rule from the requirement of anomaly cancellation states that the number of $\lambda^{a}$ 's must be given by $\int_{\Sigma} \Phi^{*} c_{1}(\mathcal{E})+r(1-g)$, where $g$ is the genus of $\Sigma$, we find that at string tree level, the number of $\lambda^{a}$ 's must be given by $r$.
} 
using the pull-back of the Levi-Civita connection on $T X$, while $D_{\bar{z}}$ is the $\bar{\partial}$ operator on $K^{1 / 2} \otimes \Phi^{*} \mathcal{E}$ using the pull-back of the connection $A$ on $\mathcal{E}$. In formulas (using a local trivialization of $\bar{K}^{1 / 2}$ and $K^{1 / 2}$, respectively), we have $^{2}$

$$
D_{z} \psi^{\bar{j}}=\partial_{z} \psi^{\bar{j}}+\Gamma_{\overline{l k}}^{\bar{j}} \partial_{z} \phi^{\bar{l}} \psi^{\bar{k}}
$$

and

$$
D_{\bar{z}} \lambda^{a}=\partial_{\bar{z}} \lambda^{a}+A^{a}{ }_{b i}(\phi) \partial_{\bar{z}} \phi^{i} \lambda^{b} .
$$

Here, $\Gamma_{\overline{l k}}^{\bar{j}}$ is the affine connection of $X$, while $A^{a}{ }_{b i}(\phi)$ is the connection on $\mathcal{E}$ in component form.

The infinitesimal transformation of the fields generated by the supercharge $\bar{Q}_{+}$under the first right-moving supersymmetry is given by

$$
\begin{aligned}
\delta \phi^{i}=0, & \delta \phi^{\bar{i}}=\bar{\epsilon}_{-} \psi^{\bar{i}}, \\
\delta \psi^{\bar{i}}=0, & \delta \psi^{i}=-\bar{\epsilon}_{-} \partial_{\bar{z}} \phi^{i}, \\
\delta \lambda^{a}=0, & \delta \lambda_{a}=\bar{\epsilon}_{-} l_{a}, \\
\delta l_{a}=0, & \delta l^{a}=\bar{\epsilon}_{-}\left(D_{\bar{z}} \lambda^{a}+F_{b i \bar{j}}^{a}(\phi) \lambda^{b} \psi^{i} \psi^{\bar{j}}\right),
\end{aligned}
$$

while the infinitesimal transformation of the fields generated by the supercharge $Q_{+}$under the second right-moving supersymmetry is given by

$$
\begin{array}{cl}
\delta \phi^{i}=\epsilon_{-} \psi^{i}, & \delta \lambda^{a}=\epsilon_{-}\left(l^{a}+A_{b i}^{a}(\phi) \lambda^{b} \psi^{i}\right), \\
\delta \psi^{i}=0, & \delta l^{a}=-\epsilon_{-} A_{b i}^{a}(\phi) l^{b} \psi^{i}, \\
\delta \phi^{\bar{i}}=0, & \delta \psi^{\bar{i}}=-\epsilon_{-} \partial_{\bar{z}} \phi^{\bar{i}}, \\
\delta \lambda_{a}=0, & \delta l_{a}=\epsilon_{-} \partial_{\bar{z}} \lambda_{a},
\end{array}
$$

where $\epsilon_{-}$and $\bar{\epsilon}_{-}$are anti-holomorphic sections of $\bar{K}^{-1 / 2}$. Since we are considering a holomorphic vector bundle $\mathcal{E}$, the supersymmetry algebra is trivially satisfied. ${ }^{3}$

\subsection{Twisting the model}

Classically, action (2.1), and therefore the model that it describes, possesses a left-moving flavour symmetry and a right-moving R-symmetry, giving rise

\footnotetext{
${ }^{2}$ Note that we have used a flat metric and hence vanishing spin connection on the Riemann surface $\Sigma$ in writing these formulas.

${ }^{3}$ The supersymmetry algebra is satisfied, provided the $(2,0)$ part of the curvature vanishes, i.e., $A_{b[i, j]}^{a}-A^{a}{ }_{c[i} A^{c}{ }_{b j]}=0$. For a real gauge field $A$ such that $A_{i}^{\dagger}=A_{i}$, this just means that $\mathcal{E}$ must be a holomorphic vector bundle [18].
} 
to a $U(1)_{\mathrm{L}} \times U(1)_{\mathrm{R}}$ global symmetry group. Denoting $\left(q_{\mathrm{L}}, q_{\mathrm{R}}\right)$ to be the leftand right-moving charges of the fields under this symmetry group, we find that $\lambda_{a}$ and $\lambda^{a}$ have charges $( \pm 1,0), \psi^{\bar{i}}$ and $\psi^{i}$ have charges $(0, \pm 1)$, and $l_{a}$ and $l^{a}$ have charges $( \pm 1, \pm 1)$, respectively. Quantum mechanically however, these symmetries are anomalous because of non-perturbative worldsheet instantons; the charge violations for the left- and right-moving global symmetries are given by $\Delta q_{\mathrm{L}}=\int_{\Sigma} \Phi^{*} c_{1}(\mathcal{E})$ and $\Delta q_{\mathrm{R}}=\int_{\Sigma} \Phi^{*} c_{1}(T X)$, respectively.

In order to define a twisted variant of the model, the spins of the various fields need to be shifted by a linear combination of their corresponding leftand right-moving charges $\left(q_{\mathrm{L}}, q_{\mathrm{R}}\right)$ under the global $U(1)_{\mathrm{L}} \times U(1)_{\mathrm{R}}$ symmetry group; by considering a shift in the spin $S$ via $S \rightarrow S+\frac{1}{2}\left[(1-2 s) q_{\mathrm{L}}\right.$ $\left.+(2 \bar{s}-1) q_{\mathrm{R}}\right]$ (where $s$ and $\bar{s}$ are real numbers), the various fields of the twisted model will transform as smooth sections of the following bundles:

$$
\begin{aligned}
\lambda_{a} \in \Gamma\left(K^{(1-s)} \otimes \Phi^{*} \mathcal{E}^{*}\right), & \lambda^{a} \in \Gamma\left(K^{s} \otimes \Phi^{*} \mathcal{E}\right), \\
\psi^{i} \in \Gamma\left(\bar{K}^{(1-\bar{s})} \otimes \Phi^{*} T X\right), & \psi^{\bar{i}} \in \Gamma\left(\bar{K}^{\bar{s}} \otimes \Phi^{*} \overline{T X}\right), \\
l_{a} \in \Gamma\left(K^{(1-s)} \otimes \bar{K}^{\bar{s}} \otimes \Phi^{*} \mathcal{E}^{*}\right), & l^{a} \in \Gamma\left(K^{s} \otimes \bar{K}^{(1-\bar{s})} \otimes \Phi^{*} \mathcal{E}\right) .
\end{aligned}
$$

Notice that for $s=\bar{s}=\frac{1}{2}$, the fields transform as smooth sections of the same tensored bundles defining the original heterotic sigma model, i.e., we get back the untwisted model.

In order for a twisted model to be physically consistent, one must ensure that the new Lorentz symmetry (which has been modified from the original due to the twist) continues to be non-anomalous quantum mechanically. Note that similar to the untwisted case, the $U(1)_{\mathrm{L}}$ and $U(1)_{\mathrm{R}}$ symmetries are anomalous in the quantum theory. The charge violations on a genus- $g$ Riemann surface $\Sigma$ are given by

$$
\begin{aligned}
& \Delta q_{\mathrm{L}}=r(1-2 s)(1-g)+\int_{\Sigma} \Phi^{*} c_{1}(\mathcal{E}), \\
& \Delta q_{\mathrm{R}}=n(2 \bar{s}-1)(g-1)+\int_{\Sigma} \Phi^{*} c_{1}(T X) .
\end{aligned}
$$

As we will show in Section 5.3, physically consistent models must obey the condition $c_{1}(\mathcal{E})=c_{1}(T X)$. Hence, we see from (2.7) and (2.8) that an example of a non-anomalous combination of global currents that one can use to twist the model with, is $\frac{1}{2}\left(J_{\mathrm{L}}-J_{\mathrm{R}}\right)$, where $s=\bar{s}=0$. If one has the additional condition that $c_{1}(\mathcal{E})=c_{1}(T X)=0$, i.e., $X$ is a Calabi-Yau, one can also consider the non-anomalous current combination $\frac{1}{2}\left(J_{\mathrm{L}}+J_{\mathrm{R}}\right)$, where $s=0$ and $\bar{s}=1$. 
Note at this point that we would like to study a twisted model which can be related to the half-twisted variant of the topological A-model at the $(2,2)$ locus where $\mathcal{E}=T X$. To this end, we shall study the twisted variant of the heterotic sigma model defined by $s=\bar{s}=0$, i.e., we consider the twisted model associated with the current combination $\frac{1}{2}\left(J_{\mathrm{L}}-J_{\mathrm{R}}\right)$. Hence, as required, the various fields in this twisted model of interest will transform as smooth sections of the following bundles:

$$
\begin{aligned}
\lambda^{a} \in \Gamma\left(\Phi^{*} \mathcal{E}\right), & \lambda_{z a} \in \Gamma\left(K \otimes \Phi^{*} \mathcal{E}^{*}\right), \\
\psi_{\bar{z}}^{i} \in \Gamma\left(\bar{K} \otimes \Phi^{*} T X\right), & \psi^{\bar{i}} \in \Gamma\left(\Phi^{*} \overline{T X}\right), \\
l_{\bar{z}}^{a} \in \Gamma\left(\bar{K} \otimes \Phi^{*} \mathcal{E}\right), & l_{z a} \in \Gamma\left(K \otimes \Phi^{*} \mathcal{E}^{*}\right) .
\end{aligned}
$$

Notice that we have included additional indices in the above fields so as to reflect their new geometrical characteristics on $\Sigma$; fields without a $z$ or $\bar{z}$ index transform as worldsheet scalars, while fields with a $z$ or $\bar{z}$ index transform as $(1,0)$ or $(0,1)$ forms on the worldsheet. In addition, as reflected by the $a, i$, and $\bar{i}$ indices, all fields continue to be valued in the pull-back of the corresponding bundles on $X$. Thus, the action of the twisted variant of the two-dimensional heterotic sigma model is given by

$$
\begin{aligned}
S_{\text {twist }}= & \int_{\Sigma}\left|d^{2} z\right|\left(\frac{1}{2} g_{i \bar{j}}\left(\partial_{z} \phi^{i} \partial_{\bar{z}} \phi^{\bar{j}}+\partial_{\bar{z}} \phi^{i} \partial_{z} \phi^{\bar{j}}\right)+g_{i \bar{j}} \psi_{\bar{z}}^{i} D_{z} \psi^{\bar{j}}\right. \\
& \left.+\lambda_{z a} D_{\bar{z}} \lambda^{a}+F_{b i \bar{j}}^{a}(\phi) \lambda_{z a} \lambda^{b} \psi_{\bar{z}}^{i} \psi^{\bar{j}}-l_{z a} l_{\bar{z}}^{a}\right)
\end{aligned}
$$

A twisted theory is the same as an untwisted one when defined on a $\Sigma$ which is flat. Hence, locally (where one has the liberty to select a flat metric), the twisting does nothing at all. However, what happens non-locally may be non-trivial. In particular, note that globally, the supersymmetry parameters $\epsilon_{-}$and $\bar{\epsilon}_{-}$must now be interpreted as sections of different line bundles; in the twisted model, the transformation laws given by (2.4) and (2.5) are still valid, and because of the shift in the spins of the various fields, we find that for the laws to remain physically consistent, $\bar{\epsilon}_{-}$must now be a function on $\Sigma$ while $\epsilon_{-}$must be a section of the non-trivial bundle $\bar{K}^{-1}$. One can therefore canonically pick $\bar{\epsilon}_{-}$to be a constant and $\epsilon_{-}$to vanish, i.e., the twisted variant of the two-dimensional heterotic sigma model has just one canonical global fermionic symmetry generated by the supercharge $\bar{Q}_{+}$. Hence, the infinitesimal transformation of the (twisted) fields under this single canonical symmetry must read (after setting $\bar{\epsilon}_{-}$to 1 )

$$
\begin{aligned}
& \delta \phi^{i}=0, \quad \delta \phi^{\bar{i}}=\psi^{\bar{i}}, \\
& \delta \psi^{\bar{i}}=0, \quad \delta \psi_{\bar{z}}^{i}=-\partial_{\bar{z}} \phi^{i},
\end{aligned}
$$




$$
\begin{aligned}
& \delta \lambda^{a}=0, \quad \delta \lambda_{z a}=l_{z a} \\
& \delta l_{z a}=0, \quad \delta l_{\bar{z}}^{a}=\left(D_{\bar{z}} \lambda^{a}+F_{b i \bar{j}}^{a}(\phi) \lambda^{b} \psi_{\bar{z}}^{i} \psi^{\bar{j}}\right) .
\end{aligned}
$$

From the $(0,2)$ supersymmetry algebra, we have $\bar{Q}_{+}^{2}=0$. In addition, (after twisting) $\bar{Q}_{+}$transforms as a scalar. Consequently, we find that the symmetry is nilpotent i.e., $\delta^{2}=0$ (off-shell) and behaves as a BRST-like symmetry.

Note at this point that the transformation laws of (2.11) can be expressed in terms of the BRST operator $\bar{Q}_{+}$, whereby $\delta W=\left\{\bar{Q}_{+}, W\right\}$ for any field $W$. One can then show that action (2.10) can be written as

$$
S_{\text {twist }}=\int_{\Sigma}\left|d^{2} z\right|\left\{\bar{Q}_{+}, V\right\}+S_{\text {top }}
$$

where

$$
V=-g_{i \bar{j}} \psi_{\bar{z}}^{i} \partial_{z} \phi^{\bar{j}}-\lambda_{z a} l_{\bar{z}}^{a}
$$

while

$$
S_{\mathrm{top}}=\frac{1}{2} \int_{\Sigma} g_{i \bar{j}}\left(\partial_{z} \phi^{i} \partial_{\bar{z}} \phi^{\bar{j}}-\partial_{\bar{z}} \phi^{i} \partial_{z} \phi^{\bar{j}}\right)
$$

is $\int_{\Sigma} \Phi^{*}(K)$, the integral of the pull-back to $\Sigma$ of the $(1,1)$ Kähler form $K=\frac{i}{2} g_{i \bar{j}} d \phi^{i} \wedge d \phi^{\bar{j}}$.

Notice that since $\bar{Q}_{+}^{2}=0$, the first term on the RHS of (2.12) is invariant under the transformation generated by $\bar{Q}_{+}$. In addition, because $d K=0$ on a Kähler manifold, $\int_{\Sigma} \Phi^{*}(K)$ depends only on the cohomology class of $K$ and the homotopy class of $\Phi_{*}(\Sigma)$, i.e., the class of maps $\Phi$. Consequently, $S_{\text {top }}$ is a topological term, invariant under local field deformations and the transformation $\delta$. Thus, the action given in (2.12) is invariant under the BRST symmetry as required. Moreover, for the transformation laws of (2.11) to be physically consistent, $\bar{Q}_{+}$must have charge $(0,+1)$ under the global $U(1)_{\mathrm{L}} \times U(1)_{\mathrm{R}}$ gauge group. Since $V$ has a corresponding charge of $(0,-1)$, while $K$ has a zero charge, $S_{\text {twist }}$ in $(2.12)$ continues to be invariant under the $U(1)_{\mathrm{L}} \times U(1)_{\mathrm{R}}$ symmetry group at the classical level.

As mentioned in the introduction, we will be studying the twisted model in perturbation theory, where one does an expansion in the inverse of the large-radius limit. Hence, only the degree-zero maps of the term $\int_{\Sigma} \Phi^{*}(K)$ contribute to the path integral factor $\mathrm{e}^{-S_{\text {twist }}}$. Therefore, in the perturbative limit, one can set $\int_{\Sigma} \Phi^{*}(K)=0$ since $d K=0$, and the model will be independent of the Kähler structure of $X$. This also means that one is free to study an equivalent action obtained by setting $S_{\text {top }}$ in (2.12) to zero. After eliminating the $l_{z a} l_{\bar{z}}^{a}$ term via its own equation of motion $l_{\bar{z}}^{a}=0$, the 
equivalent action in perturbation theory reads

$$
S_{\text {pert }}=\int_{\Sigma}\left|d^{2} z\right|\left(g_{i \bar{j}} \partial_{z} \phi^{\bar{j}} \partial_{\bar{z}} \phi^{i}+g_{i \bar{j}} \psi_{\bar{z}}^{i} D_{z} \psi^{\bar{j}}+\lambda_{z a} D_{\bar{z}} \lambda^{a}+F^{a}{ }_{b i \bar{j}} \lambda_{z a} \lambda^{b} \psi_{\bar{z}}^{i} \psi^{\bar{j}}\right),
$$

where it can also written as

$$
S_{\text {pert }}=\int_{\Sigma}\left|d^{2} z\right|\left\{\bar{Q}_{+}, V\right\}
$$

Note that the original symmetries of the theory persist despite limiting ourselves to the perturbation theory; even though $S_{\text {top }}=0$, from (2.16), one finds that $S_{\text {pert }}$ is invariant under the nilpotent BRST symmetry generated by $\bar{Q}_{+}$. It is also invariant under the $U(1)_{\mathrm{L}} \times U(1)_{\mathrm{R}}$ global symmetry. $S_{\text {pert }}$ shall henceforth be the action of interest in all our subsequent discussions.

\section{Chiral algebras from the twisted heterotic sigma model}

\subsection{The chiral algebra}

Classically, the model is conformally invariant. The trace of the stress tensor from $S_{\text {pert }}$ vanishes, i.e., $T_{z \bar{z}}=0$. The other non-zero components of the stress tensor, at the classical level, are given by

$$
T_{z z}=g_{i \bar{j}} \partial_{z} \phi^{i} \partial_{z} \phi^{\bar{j}}+\lambda_{z a} D_{z} \lambda^{a}
$$

and

$$
T_{\bar{z} \bar{z}}=g_{i \bar{j}} \partial_{\bar{z}} \phi^{i} \partial_{\bar{z}} \phi^{\bar{j}}+g_{i \bar{j}} \psi_{\bar{z}}^{i}\left(\partial_{\bar{z}} \psi^{\bar{j}}+\Gamma_{\overline{l k}}^{\bar{j}} \partial_{\bar{z}} \phi^{\bar{l}} \psi^{\bar{k}}\right) .
$$

Furthermore, one can go on to show that

$$
T_{\bar{z} \bar{z}}=\left\{\bar{Q}_{+},-g_{i \bar{j}} \psi_{\bar{z}}^{i} \partial_{\bar{z}} \phi^{\bar{j}}\right\},
$$

and

$$
\begin{aligned}
{\left[\bar{Q}_{+}, T_{z z}\right] } & =l_{z a} D_{z} \lambda^{a}+\left(g_{i \bar{j}} D_{z} \psi^{\bar{j}}+F_{b i \bar{j}}^{a}(\phi) \lambda_{z a} \lambda^{b} \psi^{\bar{j}}\right) \partial_{z} \phi^{i} \\
& =0 \text { (on - shell }) .
\end{aligned}
$$

From (3.4) and (3.3), we see that all components of the stress tensor are $\bar{Q}_{+^{-}}$ invariant; $T_{z z}$ is an operator in the $\bar{Q}_{+}$-cohomology while $T_{\bar{z} \bar{z}}$ is $\bar{Q}_{+}$-exact and thus trivial in $\bar{Q}_{+}$-cohomology. The fact that $T_{z z}$ is not $\bar{Q}_{+}$-exact even at the classical level implies that the twisted model is not a $2 \mathrm{D}$ topological field theory; rather, it is a $2 \mathrm{D}$ conformal field theory. This is because the original model has $(0,2)$ and not $(2,2)$ supersymmetry. On the other hand, the fact that $T_{\bar{z} \bar{z}}$ is $\bar{Q}_{+}$-exact has some non-trivial consequences on the nature of the local operators in the $\bar{Q}_{+}$-cohomology. Let us discuss this further. 
We say that a local operator $\mathcal{O}$ inserted at the origin has dimension $(n, m)$ if under a rescaling $z \rightarrow \lambda z, \bar{z} \rightarrow \bar{\lambda} z$ (which is a conformal symmetry of the classical theory), it transforms as $\partial^{n+m} / \partial z^{n} \partial \bar{z}^{m}$, that is, as $\lambda^{-n} \bar{\lambda}^{-m}$. Classical local operators have dimensions $(n, m)$, where $n$ and $m$ are nonnegative integers. ${ }^{4}$ However, only local operators with $m=0$ survive in $\bar{Q}_{+}$-cohomology. The reason for the last statement is that the rescaling of $\bar{z}$ is generated by $\bar{L}_{0}=\oint d \bar{z} \bar{z} T_{\bar{z} \bar{z}}$. As we noted in the previous paragraph, $T_{\bar{z} \bar{z}}$ is of the form $\left\{\bar{Q}_{+}, \ldots\right\}$, so $\bar{L}_{0}=\left\{\bar{Q}_{+}, V_{0}\right\}$ for some $V_{0}$. If $\mathcal{O}$ is to be admissible as a local physical operator, it must at least be true that $\left\{\bar{Q}_{+}, \mathcal{O}\right\}=0$. Consequently, $\left[\bar{L}_{0}, \mathcal{O}\right]=\left\{\bar{Q}_{+},\left[V_{0}, \mathcal{O}\right]\right\}$. Since the eigenvalue of $\bar{L}_{0}$ on $\mathcal{O}$ is $m$, we have $\left[\bar{L}_{0}, \mathcal{O}\right]=m \mathcal{O}$. Therefore, if $m \neq 0$, it follows that $\mathcal{O}$ is $\bar{Q}_{+}$-exact and thus trivial in $\bar{Q}_{+}$-cohomology.

By a similar argument, we can show that $\mathcal{O}$, as an element of the $\bar{Q}_{+^{-}}$ cohomology, varies holomorphically with $z$. Indeed, since the momentum operator (which acts on $\mathcal{O}$ as $\partial_{\bar{z}}$ ) is given by $\bar{L}_{-1}$, the term $\partial_{\bar{z}} \mathcal{O}$ will be given by the commutator $\left[\bar{L}_{-1}, \mathcal{O}\right]$. Since $\bar{L}_{-1}=\oint d \bar{z} T_{\bar{z} \bar{z}}$, we will have $\bar{L}_{-1}=\left\{\bar{Q}_{+}, V_{-1}\right\}$ for some $V_{-1}$. Hence, because $\mathcal{O}$ is physical such that $\left\{\bar{Q}_{+}, \mathcal{O}\right\}=0$, it will be true that $\partial_{\bar{z}} \mathcal{O}=\left\{\bar{Q}_{+},\left[V_{-1}, \mathcal{O}\right]\right\}$ and thus vanishes in $\bar{Q}_{+}$-cohomology.

The observations that we have so far are based solely on classical grounds. The question that one might then ask is whether these observations will continue to hold when we eventually consider the quantum theory. The key point to note is that if it is true classically that a cohomology vanishes, it should continue to do so in perturbation theory, when quantum effects are small enough. Since the above observations were made based on the classical fact that $T_{\bar{z} \bar{z}}$ vanishes in $\bar{Q}_{+}$-cohomology, they will continue to hold at the quantum level. Let us look at the quantum theory more closely.

\subsubsection{The quantum theory}

Quantum mechanically, the conformal structure of the theory is violated by a non-zero 1-loop $\beta$-function; renormalization adds to the classical action $S_{\text {pert }}$ a term of the form:

$$
\Delta_{1 \text {-loop }}=c_{1} R_{i \bar{j}} \partial_{z} \phi^{\bar{j}} \psi_{\bar{z}}^{i}+c_{2} g^{i \bar{j}} F_{b i \bar{j}}^{a} \lambda_{z a} l_{\bar{z}}^{b}
$$

for some divergent constants $c_{1,2}$, where $R_{i \bar{j}}$ is the Ricci tensor of $X$. In the Calabi-Yau case, one can choose a Ricci-flat metric and a solution to the Uhlenbeck-Yau equation, $g^{i \bar{j}} F_{b i \bar{j}}^{a}=0$, such that $\Delta_{1-\text { loop }}$ vanishes and

\footnotetext{
${ }^{4}$ Anomalous dimensions under RG flow may shift the values of $n$ and $m$ quantum mechanically, but the spin given by $(n-m)$, being an intrinsic property, remains unchanged.
} 
the original action is restored. In this case, the classical observations made above continue to hold true. On the other hand, in the "massive models" where $c_{1}(X) \neq 0$, there is no way to set $\Delta_{1 \text {-loop }}$ to zero. Conformal invariance is necessarily lost, and there is non-trivial RG running. However, one can continue to express $T_{\bar{z} \bar{z}}$ as $\left\{\bar{Q}_{+}, \ldots\right\}$, i.e., it remains $\bar{Q}_{+}$-exact and thus continues to vanish in $\bar{Q}_{+}$-cohomology. Hence, the above observations about the holomorphic nature of the local operators having dimension $(n, 0)$ continue to hold in the quantum theory.

We would also like to bring to the reader's attention another important feature of the $\bar{Q}_{+}$-cohomology at the quantum level. Recall that classically, we had $\left[\bar{Q}_{+}, T_{z z}\right]=0$ via the classical equations of motion. Notice that the classical expression for $T_{z z}$ is not modified at the quantum level (at least up to 1-loop), since even in the non-Calabi-Yau case, the additional term of $\Delta_{1 \text {-loop }}$ in the quantum action does not contribute to $T_{z z}$. However, due to 1-loop corrections to the action of $\bar{Q}_{+}$, we have, at the quantum level

$$
\left[\bar{Q}_{+}, T_{z z}\right]=\partial_{z}\left(R_{i \bar{j}} \partial_{z} \phi^{i} \psi^{\bar{j}}\right)+\cdots
$$

(where '...' is also a partial derivative of some terms with respect to $z$ ). Note that the term on the RHS of (3.6) cannot be eliminated through the equations of motion in the quantum theory. Neither can we modify $T_{z z}$ (by subtracting a total derivative term) such that it continues to be $\bar{Q}_{+}$-invariant. This implies that in a 'massive' model, operators do not remain in the $\bar{Q}_{+}$-cohomology after general holomorphic coordinate transformations on the worldsheet, i.e., the model is not conformal at the level of the $\bar{Q}_{+}$-cohomology. ${ }^{5}$ However, $T_{z z}$ continues to be holomorphic in $z$ up to $\bar{Q}_{+}$-trivial terms; from the conservation of the stress tensor, we have $\partial_{\bar{z}} T_{z z}=-\partial_{z} T_{z \bar{z}}$, and $T_{z \bar{z}}$, while no longer zero, is now given by $T_{z \bar{z}}=\left\{\bar{Q}_{+}, G_{z \bar{z}}\right\}$ for some $G_{z \bar{z}}$, i.e., $\partial_{z} T_{z \bar{z}}$ continues to be $\bar{Q}_{+}$-exact, and $\partial_{\bar{z}} T_{z z} \sim 0$ in $\bar{Q}_{+}$-cohomology. The holomorphy of $T_{z z}$, together with relation (3.6), has further implications for the $\bar{Q}_{+}$-cohomology of local operators; by a Laurent expansion of $T_{z z},{ }^{6}$ one can use (3.6) to show that $\left[\bar{Q}_{+}, L_{-1}\right]=0$. This means that operators remain in the $\bar{Q}_{+}$-cohomology after global translations on the worldsheet. In addition, recall that $\bar{Q}_{+}$is a scalar with spin zero in the twisted model. As shown few paragraphs before, we have the condition $\bar{L}_{0}=0$. Let the spin be $S$, where $S=L_{0}-\bar{L}_{0}$. Therefore,

\footnotetext{
${ }^{5}$ In Section 5.7, we will examine more closely, from a different point of view, the 1-loop correction to the action of $\bar{Q}_{+}$associated with the beta-function, where (3.6) will appear in a different guise.

${ }^{6}$ Since we are working modulo $\bar{Q}_{+}$-trivial operators, it suffices for $T_{z z}$ to be holomorphic up to $\bar{Q}_{+}$-trivial terms before an expansion in terms of Laurent coefficients is permitted.
} 
$\left[\bar{Q}_{+}, S\right]=0$ implies that $\left[\bar{Q}_{+}, L_{0}\right]=0$. In other words, operators remain in the $\bar{Q}_{+}$-cohomology after global dilatations of the worldsheet coordinates.

One can also make the following observations about the correlation functions of these local operators. First, note that $\left\langle\left\{\bar{Q}_{+}, W\right\}\right\rangle=0$ for any $W$ and recall that for any local physical operator $\mathcal{O}_{\alpha}$, we have $\left\{\bar{Q}_{+}, \mathcal{O}_{\alpha}\right\}=0$. Since the $\partial_{\bar{z}}$ operator on $\Sigma$ is given by $\bar{L}_{-1}=\oint d \bar{z} T_{\bar{z} \bar{z}}$, where $T_{\bar{z} \bar{z}}=\left\{\bar{Q}_{+}, \cdots\right\}$, we find that $\partial_{\bar{z}}\left\langle\mathcal{O}_{1}\left(z_{1}\right) \mathcal{O}_{2}\left(z_{2}\right) \cdots \mathcal{O}_{s}\left(z_{s}\right)\right\rangle$ is given by $\oint d \bar{z}\left\langle\left\{\bar{Q}_{+}, \cdots\right\} \mathcal{O}_{1}\left(z_{1}\right)\right.$ $\left.\mathcal{O}_{2}\left(z_{2}\right) \cdots \mathcal{O}_{s}\left(z_{s}\right)\right\rangle=\oint d \bar{z}\left\langle\left\{\bar{Q}_{+}, \cdots \prod_{i} \mathcal{O}_{i}\left(z_{i}\right)\right\}\right\rangle=0$. Thus, the correlation functions are always holomorphic in $z$. Secondly, $T_{z \bar{z}}=\left\{\bar{Q}_{+}, G_{z \bar{z}}\right\}$ for some $G_{z \bar{z}}$ in the "massive" models. Hence, the variation of the correlation functions due to a change in the scale of $\Sigma$ will be given by $\left\langle\mathcal{O}_{1}\left(z_{1}\right) \mathcal{O}_{2}\left(z_{2}\right) \cdots\right.$ $\left.\mathcal{O}_{s}\left(z_{s}\right)\left\{\bar{Q}_{+}, G_{z \bar{z}}\right\}\right\rangle=\left\langle\left\{\bar{Q}_{+}, \prod_{i} \mathcal{O}_{i}\left(z_{i}\right) \cdot G_{z \bar{z}}\right\}\right\rangle=0$. In other words, the correlation functions of local physical operators will continue to be invariant under arbitrary scalings of $\Sigma$. Thus, the correlation functions are always independent of the Kähler structure on $\Sigma$ and depend only on its complex structure.

\subsubsection{A holomorphic chiral algebra $\mathcal{A}$}

Let $\mathcal{O}(z)$ and $\widetilde{\mathcal{O}}\left(z^{\prime}\right)$ be two $\bar{Q}_{+}$-closed operators such that their product is $\bar{Q}_{+}$-closed as well. Now, consider their operator product expansion (OPE)

$$
\mathcal{O}(z) \widetilde{\mathcal{O}}\left(z^{\prime}\right) \sim \sum_{k} f_{k}\left(z-z^{\prime}\right) \mathcal{O}_{k}\left(z^{\prime}\right)
$$

in which the explicit form of the coefficients $f_{k}$ must be such that the scaling dimensions and $U(1)_{\mathrm{L}} \times U(1)_{\mathrm{R}}$ charges of the operators agree on both sides of the OPE. In general, $f_{k}$ is not holomorphic in $z$. However, if we work modulo $\bar{Q}_{+}$-exact operators in passing to the $\bar{Q}_{+}$-cohomology, the $f_{k}$ 's which are non-holomorphic and are thus not annihilated by $\partial / \partial \bar{z}$ drop out from the OPE because they multiply operators $\mathcal{O}_{k}$ which are $\bar{Q}_{+}$-exact. This is true because $\partial / \partial \bar{z}$ acts on the LHS of (3.7) to give terms which are cohomologically trivial. ${ }^{7}$ In other words, we can take the $f_{k}$ 's to be holomorphic coefficients in studying the $\bar{Q}_{+}$-cohomology. Thus, the OPE of (3.7) has a holomorphic structure.

In summary, we have established that the $\bar{Q}_{+}$-cohomology of holomorphic local operators has a natural structure of a holomorphic chiral algebra (as defined in the mathematical literature), which we shall henceforth call $\mathcal{A}$; it is always preserved under global translations and dilatations, though (unlike

\footnotetext{
${ }^{7}$ Since $\left\{\bar{Q}_{+}, \mathcal{O}\right\}=0$, we have $\partial_{\bar{z}} \mathcal{O}=\left\{\bar{Q}_{+}, V(z)\right\}$ for some $V(z)$, as argued before. Hence $\partial_{\bar{z}} \mathcal{O}(z) \cdot \widetilde{\mathcal{O}}\left(z^{\prime}\right)=\left\{\bar{Q}_{+}, V(z) \widetilde{\mathcal{O}}\left(z^{\prime}\right)\right\}$.
} 
the usual physical notion of a chiral algebra) it may not be preserved under general holomorphic coordinate transformations on the Riemann surface $\Sigma$. Likewise, the OPEs of the chiral algebra of local operators obey the usual relations of holomorphy, associativity, and invariance under translations and scalings of $z$, but not necessarily invariance under arbitrary holomorphic reparameterizations of $z$. The local operators are of dimension $(n, 0)$ for $n \geq$ 0 , and the chiral algebra of such operators requires a flat metric up to scaling on $\Sigma$ to be defined. ${ }^{8}$ Therefore, the chiral algebra that we have obtained can only be globally defined on a Riemann surface of genus 1, or be locally defined on an arbitrary but curved $\Sigma$. To define the chiral algebra globally on a surface of higher genus requires more in-depth analysis and is potentially obstructed by an anomaly involving $c_{1}(\Sigma)$ and $\left(c_{1}(\mathcal{E})-c_{1}(X)\right)$, which we will discuss in Sections 4 and 5.6. Last but not least, as is familiar for chiral algebras, the correlation functions of these operators depend on $\Sigma$ only via its complex structure. The correlation functions are holomorphic in the parameters of the theory and are therefore protected from perturbative corrections.

\subsection{The moduli of the chiral algebra}

Here, we shall consider the moduli of the chiral algebra $\mathcal{A}$. To this end, let us first make some additional observations about $\mathcal{A}$.

First, notice that the metric $g_{i \bar{j}}$ of the target space $X$ appears in the classical action $S_{\text {pert }}$ inside a term of the form $\left\{\bar{Q}_{+}, \cdots\right\}$. Similarly, the fibre metric $h_{a \bar{b}}$ of the holomorphic vector bundle $\mathcal{E}$, which appears implicitly in the expression $-\lambda_{z a} l_{\bar{z}}^{a}$ of $V$ in (2.13), also sits inside a term of the form $\left\{\bar{Q}_{+}, \cdots\right\}$. Hence, in passing to the $\bar{Q}_{+}$-cohomology, we find that the chiral algebra is independent of the metrics on $X$ and the fibre space of $\mathcal{E}$.

Secondly, note that the chiral algebra does depend on the complex structure of $X$ and the holomorphic structure of $\mathcal{E}$ because they enter in the definition of the fields and the fermionic symmetry transformation laws of (2.11). As we are not going to study how the chiral algebra behaves under a continuous deformation of the bundle $\mathcal{E}$, its dependence on the holomorphic structure of $\mathcal{E}$ shall be irrelevant to us, at least in this paper. Note also that the chiral algebra varies holomorphically with the complex structure of $X$; one can show, using the form of $S_{\text {pert }}$ in (2.16), that if $J$ denotes the complex structure of $X$, an anti-holomorphic derivative $\partial / \partial \bar{J}$ changes $S_{\text {pert }}$ by a term of the form $\left\{\bar{Q}_{+}, \ldots\right\}$.

\footnotetext{
${ }^{8}$ Notice that we have implicitly assumed the flat metric on $\Sigma$ in all of our analysis thus far.
} 
We shall now consider adding to $S_{\text {pert }}$ a term which will represent the moduli of the chiral algebra $\mathcal{A}$. As we will show shortly in Section 3.3, this term results in a non-Kähler deformation of the target space $X$. Thus, $X$ will be a complex, hermitian manifold in all our following discussions.

To proceed, let $T=\frac{1}{2} T_{i j} d \phi^{i} \wedge d \phi^{j}$ be any two-form on $X$ that is of type $(2,0) .{ }^{9}$ The term that deforms $S_{\text {pert }}$ will then be given by

$$
S_{T}=\int_{\Sigma}\left|d^{2} z\right|\left\{\bar{Q}_{+}, T_{i j} \psi_{\bar{z}}^{i} \partial_{z} \phi^{j}\right\}
$$

By construction, $S_{T}$ is $\bar{Q}_{+}$-invariant. Moreover, since it has vanishing $\left(q_{\mathrm{L}}, q_{\mathrm{R}}\right)$ charges, it is also invariant under the global $U(1)_{\mathrm{L}} \times U(1)_{\mathrm{R}}$ symmetry group. Hence, as required, the addition of $S_{T}$ preserves the classical symmetries of the theory. Explicitly, we then have

$$
S_{T}=\int_{\Sigma}\left|d^{2} z\right|\left(T_{i j, \bar{k}} \psi^{\bar{k}} \psi_{\bar{z}}^{i} \partial_{z} \phi^{j}-T_{i j} \partial_{\bar{z}} \phi^{i} \partial_{z} \phi^{j}\right),
$$

where $T_{i j, \bar{k}}=\partial T_{i j} / \partial \phi^{\bar{k}}$. Note that since $\left|d^{2} z\right|=i d z \wedge d \bar{z}$, we can write the second term on the RHS of (3.9) as

$$
S_{T}^{(2)}=\frac{i}{2} \int_{\Sigma} T_{i j} d \phi^{i} \wedge d \phi^{j}=i \int_{\Sigma} \Phi^{*}(T) .
$$

Recall that in perturbation theory, we are considering degree-zero maps $\Phi$ with no multiplicity. Hence, for $S_{T}^{(2)}$ to be non-vanishing (in contrast to the closed Kähler form $K$ that we encountered in Section 2.2), $T$ must not be closed, i.e., $d T \neq 0$. In other words, one must have a non-zero flux $\mathcal{H}=d T$. As $T$ is of type $(2,0), \mathcal{H}$ will be a three-form of type $(3,0) \oplus(2,1)$.

Notice here that the first term on the RHS of (3.9) is expressed in terms of $\mathcal{H}$, since $T_{i j, \bar{k}}$ is simply the $(2,1)$ part of $\mathcal{H}$. In fact, $S_{T}^{(2)}$ can also be written in terms of $\mathcal{H}$ as follows. Suppose that $C$ is a three-manifold whose boundary is $\Sigma$ and over which the map $\Phi: \Sigma \rightarrow X$ extends. Then, if $T$ is globally defined as a $(2,0)$-form, the relation $\mathcal{H}=d T$ implies, via Stoke's theorem, that

$$
S_{T}^{(2)}=i \int_{C} \Phi^{*}(\mathcal{H})
$$

Hence, we see that $S_{T}$ can be expressed solely in terms of the three-form flux $\mathcal{H}$ (modulo terms that do not affect the perturbation theory).

Note at this point that we do not actually want to limit ourselves to the case that $T$ is globally defined; as is clear from (3.8), if $T$ were to be

\footnotetext{
${ }^{9}$ As we will see shortly, the restriction of $T$ to be a gauge field of type $(2,0)$, will enable us to associate the moduli of the chiral algebra with the moduli of sheaves of vertex superalgebras studied in the mathematical literature $[3,5]$ as desired.
} 
globally defined, $S_{T}$ and therefore the moduli of the chiral algebra would vanish in $\bar{Q}_{+}$-cohomology. Fortunately, the RHS of (3.11) makes sense as long as $\mathcal{H}$ is globally defined, with the extra condition that $\mathcal{H}$ be closed, since $C$ cannot be the boundary of a four-manifold. ${ }^{10}$ Therefore, (as will be shown shortly via Poincaré's lemma), it suffices for $T$ to be locally defined such that $\mathcal{H}=d T$ is true only locally. Hence, $T$ must be interpreted an a two-form gauge field in the string theory (or a non-trivial connection on gerbes in mathematical theories).

In the quantum theory, a shift in the (Euclidean) action $S_{\mathrm{E}}$ by an integral multiple of $2 \pi i$ is irrelevant as the path integral factor is $\mathrm{e}^{-S_{\mathrm{E}}}$. Hence, the effective range of the continuous moduli of $\mathcal{H}$ is such that $0 \leq S_{T}^{(2)}<2 \pi i$. Also, the continuous $U(1)_{\mathrm{L}}$ and $U(1)_{\mathrm{R}}$ symmetries of the classical theory reduce to discrete symmetries in the quantum theory due to worldsheet instantons. In order for the discrete symmetries to remain anomaly-free, $\frac{\mathcal{H}}{2 \pi}$ must be an integral cohomology class, i.e., $\frac{1}{2 \pi} \int_{C} \Phi^{*}(\mathcal{H}) \in \mathbb{Z}$. Hence, the continuous moduli of $\mathcal{H}$ present in perturbation theory may be absent in the non-perturbative theory. Since we are only considering the physics in the perturbative regime, we will not see this effect.

In writing $S_{T}^{(2)}$ in terms of $\mathcal{H}$, we have made the assumption that $\Phi$ extends over some three-manifold $C$ with boundary $\Sigma$. Since in perturbation theory, one considers only topologically trivial maps $\Phi$ which can be extended over any chosen $C$, the assumption is valid. Non-perturbatively however, one must also consider the contributions coming from topologically non-trivial maps as well. Thus, an extension of the map over $C$ may not exist. Therefore, the current definition of $S_{T}^{(2)}$ will not suffice. Notice also, that $T$ cannot be completely determined as a two-form gauge field by its curvature $\mathcal{H}=d T$, as one may add a flat two-form gauge field to $T$ where $\mathcal{H}$ does not change at all. This indeterminacy of $T$ is inconsequential in perturbation theory as $S_{T}^{(2)}$ can be made to depend solely on $\mathcal{H}$ via (3.11). Non-perturbatively on the other hand, because $C$ may not exist, $S_{T}^{(2)}$ can only be expressed in terms of $T$ and not $\mathcal{H}$, as in (6.9). The explicit details of $T$ will then be important. Since the sheaf of CDOs or vertex superalgebras, as defined in the mathematical literature, only depends on $\mathcal{H}$, the theory of CDOs can only be used to describe the physics of the twisted model in perturbation theory. This dependency of the sheaf of vertex superalgebras on $\mathcal{H}$ is also what motivates us to express $S_{T}$ entirely in terms of $\mathcal{H}$.

\footnotetext{
${ }^{10}$ From homology theory, the boundary of a boundary is empty. Hence, since $\Sigma$ exists as the boundary of $C$, the three-manifold $C$ itself cannot be a boundary of a higher dimensional four-manifold.
} 


\subsubsection{Moduli}

As mentioned earlier, $T$ must be locally defined only such that the expression $\mathcal{H}=d T$ is valid only locally. At the same time, $\mathcal{H}$ must be globally defined and closed so that (3.11) can be consistent. Fortunately, one can show that a globally defined, closed three-form $\mathcal{H}$ of type $(3,0) \oplus(2,1)$ can be expressed locally as $\mathcal{H}=d T$, where $T$ is a locally defined two-form of type $(2,0)$. To demonstrate this, let us first select any local two-form $Y$ such that $\mathcal{H}=d Y$. Poincaré's lemma asserts that $Y$ will exist because $\mathcal{H}$ is closed and globally defined. In general, $Y$ is given by a sum of terms $Y^{(2,0)}+Y^{(1,1)}+Y^{(0,2)}$ of the stated types. Since in our application, $\mathcal{H}$ has no component of type $(0,3)$, it will mean that $\bar{\partial} Y^{(0,2)}=0$. By the $\bar{\partial}$ version of Poincaré's lemma, we then have $Y^{(0,2)}=\bar{\partial} \eta$, where $\eta$ is a one-form of type $(0,1)$. Let $\tilde{Y}=Y-d \eta$, so that we have $\mathcal{H}=d Y=d \tilde{Y}$, where $\tilde{Y}=\tilde{Y}^{(2,0)}+\tilde{Y}^{(1,1)}$. Since $\mathcal{H}$ has no component of type $(1,2)$ either, it will mean that $\bar{\partial} \tilde{Y}^{(1,1)}=0$. By the $\bar{\partial}$ version of Poincaré's lemma again, we will have $\tilde{Y}^{(1,1)}=\bar{\partial} \zeta$, where $\zeta$ is a one-form of type $(1,0)$. By defining $T=\tilde{Y}-d \zeta$, we have $\mathcal{H}=d \tilde{Y}=d T$, where $T$ is a two-form of type $(2,0)$ as promised.

Recall that if $T$ and therefore $\mathcal{H}=d T$ is globally defined, $S_{T}$ will vanish in $\bar{Q}_{+}$-cohomology. Hence, in perturbation theory, the moduli of the chiral algebra derived from the twisted heterotic sigma model on a holomorphic vector bundle $\mathcal{E}$ over a complex hermitian manifold $X$ - are parameterized by a closed three-form $\mathcal{H}$ of type $(3,0) \oplus(2,1)$ modulo forms that can be written globally as $\mathcal{H}=d T$, where $T$ is a form of type $(2,0)$. In other words, the moduli are represented by some cohomology class that $\mathcal{H}$ represents. Nonperturbatively, however, the picture can be very different; for the topologically non-trivial maps of higher degree, $S_{T}^{(2)}$ can only be expressed in terms of $T$ via (6.9), where even flat $T$ fields will be important. In addition, $\mathcal{H}$ must be an integral class, which means that the moduli in the non-perturbative theory must be discrete and not continuous. The analysis is beyond the scope of the present paper, and we shall not expound on it further.

\subsubsection{Interpretation via $H^{1}\left(X, \Omega_{X}^{2, \mathrm{cl}}\right)$}

Now, we would like to determine the type of cohomology class that $\mathcal{H}$ represents.

To this end, let $U_{a}, a=1, \ldots, s$ be a collection of small open sets providing a good cover of $X$ such that their mutual intersections are open sets as well.

Suppose that we have a globally defined closed three-form $\mathcal{H}$ of type $(3,0) \oplus(2,1)$ that can be expressed as $\mathcal{H}=d T$ locally, where $T$ is a twoform of type $(2,0)$ which is locally-defined. This means that on each $U_{a}$, 
we will have a $(2,0)$-form $T_{a}$, such that $\mathcal{H}_{a}=d T_{a}$. On each open double intersection $U_{a} \cap U_{b}$, let us define $T_{a b}=T_{a}-T_{b}$, where

$$
T_{a b}=-T_{b a}
$$

for each $a, b$, and

$$
T_{a b}+T_{b c}+T_{c a}=0
$$

for each $a, b$ and $c$. Since $\mathcal{H}$ is globally defined, $\mathcal{H}_{a}=\mathcal{H}_{b}$ on the intersection $U_{a} \cap U_{b}$, so that $d T_{a b}=0$. This implies that $\partial T_{a b}=\bar{\partial} T_{a b}=0$. Notice that since on each $U_{a}$, we have $\mathcal{H}_{a}=d T_{a}$, the shift given by $T_{a} \rightarrow T_{a}+S_{a}$, where $d S_{a}=0$ (and therefore $\partial S_{a}=\bar{\partial} S_{a}=0$ ), leaves each $\mathcal{H}_{a}$ invariant. In other words, in describing $\mathcal{H}$, we have an equivalence relation

$$
T_{a b} \sim T_{a b}^{\prime}=T_{a b}+S_{a}-S_{b} .
$$

Let us proceed to describe $T_{a b}$ more precisely. In order to do so, let us first denote $\Omega_{X}^{2}$ as the sheaf of $(2,0)$-forms on $X$ and $\Omega_{X}^{2, \text { cl }}$ as the sheaf of such forms that are annihilated by $\partial$. (The label "cl" is short for "closed" and refers to forms that are closed in the sense of being annihilated by $\partial$. We will occasionally write this as $\Omega^{2, c l}$ when there is no ambiguity.) A holomorphic section of $\Omega_{X}^{2 \text {,cl }}$ in a given set $U \subset X$ is a $(2,0)$-form on $U$ that is annihilated by both $\bar{\partial}$ and $\partial$. Likewise, $\Omega_{X}^{n, \mathrm{cl}}$ is the sheaf whose sections are $(n, 0)$ forms that are $\partial$-closed, such that its holomorphic sections are also annihilated by $\bar{\partial}$. Since it was shown in the last paragraph that each $T_{a} \in \Omega_{X}^{2}$ and that $\partial T_{a b}=\bar{\partial} T_{a b}=0$ in each double intersection $U_{a} \cap U_{b}$, we find that $T_{a b}$ must be a holomorphic section of $\Omega_{X}^{2, \mathrm{cl}}$.

Next, notice from the equivalence relation (3.14) that $T_{a b} \sim 0$ if we can express $T_{a b}=S_{b}-S_{a}$ in $U_{a} \cap U_{b}$, where $S_{a}$ and $S_{b}$ are holomorphic in each $U_{a}$ and $U_{b}$ respectively (since $\bar{\partial} S_{a}=\bar{\partial} S_{b}=0$ ). Hence, the non-vanishing $T_{a b}$ 's are those which obey the identities (3.12) and (3.13), modulo those that can be expressed as $T_{a b}=S_{b}-S_{a}$. In other words, $T_{a b}$ is an element of the Cech cohomology group $H^{1}\left(X, \Omega_{X}^{2, c l}\right)$.

Now, if $\mathcal{H}$ is globally given by the exact form $\mathcal{H}=d T$, it would mean that $T$ is globally defined and as such, $T_{a}=T_{b}=T$ in each $U_{a} \cap U_{b}$, whereupon all $T_{a b}$ 's must vanish. Thus we have obtained a map between the space of closed three-forms $\mathcal{H}$ of type $(3,0) \oplus(2,1)$, modulo forms that can be written globally as $d T$ for $T$ of type $(2,0)$, to the Cech cohomology group $H^{1}\left(X, \Omega_{X}^{2, \text { cl }}\right)$. One could have also run everything backwards, starting with an element of $H^{1}\left(X, \Omega_{X}^{2, \text { cl }}\right)$ and using the partition of unity subordinate to the cover $U_{a}$ of $X$ to construct the inverse of this map [12]. However, since this argument is also standard in the mathematical literature in relating a $\bar{\partial}$ and Cech cohomology, we shall skip it for brevity. Therefore, we can conclude 
that $\mathcal{H}$ represents an element of $H^{1}\left(X, \Omega_{X}^{2, \mathrm{cl}}\right)$. Hence, in perturbation theory, the moduli of the chiral algebra derived from the twisted heterotic sigma model on a holomorphic vector bundle $\mathcal{E}$ over a complex hermitian manifold $X$, like the moduli of sheaves of vertex superalgebras studied in the mathematical literature $[3,5]$, are associated with $H^{1}\left(X, \Omega_{X}^{2, \mathrm{cl}}\right)$.

\subsection{The moduli as a non-Kähler deformation of $X$}

As shown above, in order to incorporate the moduli so that we can obtain a family of chiral algebras, we need to turn on the three-form $\mathcal{H}$-flux. As we will show in this section, this will in turn result in a non-Kähler deformation of the target space $X$. The motivation for our present discussion rests on the fact that this observation will be important when we discuss the physical application of our results at the $(2,2)$ locus in Section 7.2.

The moduli's connection with a non-Kähler deformation of $X$ can be made manifest through the twisted heterotic sigma model's relation to a unitary model with $(0,2)$ supersymmetry. Thus, let us first review some known results $[19,20]$ about $(0,2)$ supersymmetry.

A unitary model with $(0,2)$ supersymmetry can be constructed by enlarging the worldsheet $\Sigma$ to a supermanifold $\widehat{\Sigma}$ with bosonic coordinates $z, \bar{z}$ and fermionic coordinates $\theta^{+}, \bar{\theta}^{+}$. (The "+" superscript in $\theta^{+}, \bar{\theta}^{+}$just indicates that they transform as sections of the positive chirality spin bundle of $\Sigma$.) The two supersymmetry generators that act geometrically in $\widehat{\Sigma}$ are given by

$$
\begin{aligned}
& \overline{\mathcal{Q}}_{+}=\frac{\partial}{\partial \bar{\theta}^{+}}-i \theta^{+} \frac{\partial}{\partial \bar{z}} \\
& \mathcal{Q}_{+}=\frac{\partial}{\partial \theta^{+}}-i \bar{\theta}^{+} \frac{\partial}{\partial \bar{z}}
\end{aligned}
$$

where $\mathcal{Q}_{+}^{2}=\overline{\mathcal{Q}}_{+}^{2}=0$ and $\left\{\mathcal{Q}_{+}, \overline{\mathcal{Q}}_{+}\right\}=-2 i \partial / \partial \bar{z}$. To construct supersymmetric Lagrangians that are invariant under $\mathcal{Q}_{+}$and $\overline{\mathcal{Q}}_{+}$, we note the fact that these operators anti-commute with the supersymmetric derivatives

$$
\begin{aligned}
& \bar{D}_{+}=\frac{\partial}{\partial \bar{\theta}^{+}}+i \theta^{+} \frac{\partial}{\partial \bar{z}} \\
& D_{+}=\frac{\partial}{\partial \theta^{+}}+i \bar{\theta}^{+} \frac{\partial}{\partial \bar{z}}
\end{aligned}
$$


and commute with $\partial_{z}$ and $\partial_{\bar{z}}$. Moreover, the measure $\left|d^{2} z\right| d \theta d \bar{\theta}$ is also supersymmetric, i.e., it is invariant under the action of $\mathcal{Q}_{+}$and $\overline{\mathcal{Q}}_{+}$. Consequently, any action constructed using only the superfields and their supersymmetric and/or partial derivatives, together with the measure, will be supersymmetric.

To construct such an action, we can describe the theory using "chiral superfields" $\Phi$ in the supermanifold $\widehat{\Sigma}$, which obey $\bar{D}_{+} \Phi^{i}=D_{+} \bar{\Phi}^{\bar{i}}=0$. They can be expanded as

$$
\begin{aligned}
& \Phi^{i}=\phi^{i}+\sqrt{2} \theta^{+} \rho_{+}^{i}-i \bar{\theta}^{+} \theta^{+} \partial_{\bar{z}} \phi^{i}, \\
& \bar{\Phi}^{\bar{i}}=\phi^{\bar{i}}-\sqrt{2} \bar{\theta}^{+} \rho_{+}^{\bar{i}}+i \bar{\theta}^{+} \theta^{+} \partial_{\bar{z}} \phi^{\bar{i}} .
\end{aligned}
$$

Here, $\phi^{i}$ and $\phi^{\bar{i}}$ are scalar fields on $\Sigma$ which define a map $\phi: \Sigma \rightarrow X$; they serve as the (local) holomorphic and anti-holomorphic complex coordinates on $X$, respectively. $\rho_{+}^{i}$ and $\rho_{+}^{\bar{i}}$ are the fermionic superpartners of the $\phi$ fields on $\Sigma$ with positive chirality, and they transform as sections of the pull-backs $\phi^{*}(T X)$ and $\phi^{*}(\overline{T X})$, respectively.

To ascertain how the various component fields of $\Phi$ transform under the two supersymmetries generated by $\overline{\mathcal{Q}}_{+}$and $\mathcal{Q}_{+}$, we must compute how the $\Phi$ superfields transform under the action of the $\overline{\mathcal{Q}}_{+}$and $\mathcal{Q}_{+}$operators defined in (3.15) and compare its corresponding components with that in the original superfields. In particular, $\overline{\mathcal{Q}}_{+}$generates the field transformations

$$
\begin{aligned}
\delta \phi^{i} & =0, \\
\delta \phi^{\bar{i}} & =-\sqrt{2} \rho_{+}^{\bar{i}}, \\
\delta \rho_{+}^{i} & =-i \sqrt{2} \partial_{\bar{z}} \phi^{i}, \\
\delta \rho_{+}^{\bar{i}} & =0 .
\end{aligned}
$$

Notice at this point that the non-zero transformations in equation (2.11) of Section 2.2 are given by $\delta \phi^{\bar{i}}=\psi^{\bar{i}}$ and $\delta \psi_{\bar{z}}^{i}=-\partial_{\bar{z}} \phi^{i}$ on-shell. These coincide with the non-zero transformations in (3.18) if we set $\psi^{\bar{i}}=-\sqrt{2} \rho_{+}^{\bar{i}}$ and $\psi_{\bar{z}}^{i}=$ $-i \rho_{+}^{i} / \sqrt{2}$. Hence, we see that the structure in a unitary $(0,2)$ model is a specialization of the structure in the twisted heterotic sigma model studied in Section 2.2, with $\overline{\mathcal{Q}}_{+}$corresponding to $\bar{Q}_{+}$. This should not be surprising since we started off with a heterotic sigma model with $(0,2)$ supersymmetry anyway. As our following arguments do not require us to refer to the field transformations generated by the other supercharge $\mathcal{Q}_{+}$, we shall omit them for brevity. 
Now let $K=K_{i}\left(\phi^{j}, \phi^{\bar{j}}\right) d \phi^{i}$ be a $(1,0)$-form on $X$, with complex conjugate $\bar{K}=\bar{K}_{\bar{i}}\left(\phi^{j}, \phi^{\bar{j}}\right) d \phi^{\bar{i}}$. A supersymmetric action can then be written as [20]

$$
S=\int\left|d^{2} z\right| d \bar{\theta}^{+} d \theta^{+}\left(-\frac{i}{2} K_{i}(\Phi, \bar{\Phi}) \partial_{z} \Phi^{i}+\frac{i}{2} \bar{K}_{\bar{i}}(\Phi, \bar{\Phi}) \partial_{z} \bar{\Phi}^{\bar{i}}\right) .
$$

Note that action density of (3.19) must be a local expression, so that the action will be invariant under additional transformations of $K$, whence one can obtain the required invariant geometrical objects that can then be globally defined. In particular, since the action density of (3.19) is a local expression, we are free to discard exact forms after integrating parts. This can be shown via a superspace extension of Poincaré's lemma. ${ }^{11}$ Consequently, the transformations of $K$ and $\bar{K}$ in the target space $X$, which correspond to transformations in superspace that leave the action invariant, will be given by

$$
K \longrightarrow K+\partial \Lambda, \quad \bar{K} \longrightarrow \bar{K}-\bar{\partial} \Lambda,
$$

where $\Lambda=\Lambda\left(\phi^{i}, \phi^{\bar{i}}\right)$ is some imaginary zero-form. In fact, under (3.20), the corresponding superfield transformations will be given by

$K_{i}(\Phi, \bar{\Phi}) \longrightarrow K_{i}(\Phi, \bar{\Phi})+\partial_{\Phi^{i}} \Lambda(\Phi, \bar{\Phi}), \bar{K}_{\bar{i}}(\Phi, \bar{\Phi}) \longrightarrow \bar{K}_{\bar{i}}(\Phi, \bar{\Phi})-\bar{\partial}_{\bar{\Phi}^{i}} \Lambda(\Phi, \bar{\Phi})$,

and one can show that the action density changes by the total derivative $-i \partial_{z} \Lambda(\Phi, \bar{\Phi})$, which can be integrated to zero.

By integrating the $\bar{\theta}^{+}$and $\theta^{+}$variables in (3.19), we get an action given by an integral over the $z$ and $\bar{z}$ variables of a Lagrangian written in terms of fields on $\Sigma$. Of particular interest would be the hermitian metric of $X$ found in the Lagrangian and defined by $d s^{2}=g_{i \bar{j}} d \phi^{i} d \phi^{\bar{j}}$. It is given by

$$
g_{i \bar{j}}=\partial_{i} \bar{K}_{\bar{j}}+\partial_{\bar{j}} K_{i} \text {. }
$$

Notice that it is invariant under (3.20); hence, it can be globally defined. Associate to this metric is a globally defined $(1,1)$-form on $X$ that is invariant under (3.20) and given by

$$
\omega_{T}=\frac{i}{2}(\bar{\partial} K-\partial \bar{K}) .
$$

Note that $\omega_{T}$ is the analogue of a Kähler $(1,1)$-form $\omega$ on a Kähler manifold. However, from (3.23), we find that in contrast to $\omega$, which obeys $\partial \omega=\bar{\partial} \omega=$

\footnotetext{
${ }^{11}$ Via a superspace extension of Poincare's lemma, we learn that locally exact forms $\widehat{Y}$ on $\widehat{\Sigma}$ are globally closed. Let $\widehat{\Sigma}$ be the boundary of a higher dimensional supermanifold $\widehat{C}$. Hence, via Stoke's theorem in superspace, we find that $\int_{\widehat{\Sigma}} \widehat{Y}=\int_{\widehat{C}} d \widehat{Y}=0$, where $d$ is the exterior derivative operator in superspace.
} 
$0, \omega_{T}$ obeys the weaker condition

$$
\bar{\partial} \partial \omega_{T}=0
$$

instead. This just reflects the well-known fact that the target space of a model with $(0,2)$ supersymmetry is in general hermitian and non-Kähler.

In order to relate the twisted heterotic sigma model to the unitary model reviewed here, we will need to express (3.19) in the form $\int\left|d^{2} z\right|\left\{\bar{Q}_{+}, V\right\}$ that $S_{\text {pert }}$ takes in Section 2.2. To do so, we must first convert (3.19) to an integral of an ordinary Lagrangian over $z$ and $\bar{z}$. The standard way to do this is to perform the integral over $\theta$ and $\bar{\theta}$. However, a convenient shortcut is to note that for any superfield $W$, we can make the replacement

$$
\begin{aligned}
\int\left|d^{2} z\right| d \bar{\theta}^{+} d \theta^{+} W & =\left.\int\left|d^{2} z\right| \frac{\partial^{2}}{\partial \bar{\theta}^{+} \partial \theta^{+}} W\right|_{\bar{\theta}^{+}=\theta^{+}=0} \\
& =\left.\int\left|d^{2} z\right| \bar{D}_{+} D_{+} W\right|_{\theta^{+}=\bar{\theta}^{+}=0} .
\end{aligned}
$$

The rationale for the first step is that for a fermionic variable $\theta, \int d \theta W=$ $\left.(\partial W / \partial \theta)\right|_{\theta=0}$. The rationale for the second step is that the $D$ 's differ from the $\partial / \partial \theta$ 's by $\partial_{\bar{z}}$ terms, which vanish upon integration by parts. Now, since $\overline{\mathcal{Q}}_{+}$differs from $\bar{D}_{+}$and $\partial / \partial \bar{\theta}^{+}$by a total derivative, we can rewrite $(3.25)$ and therefore action (3.19) as

$$
S=\left.\int\left|d^{2} z\right|\left\{\overline{\mathcal{Q}}_{+},\left[D_{+}, W\right]\right\}\right|_{\theta^{+}=\bar{\theta}^{+}=0},
$$

where $W=-\frac{i}{2} K_{i}(\Phi, \bar{\Phi}) \partial_{z} \Phi^{i}+\frac{i}{2} \bar{K}_{\bar{i}}(\Phi, \bar{\Phi}) \partial_{z} \Phi^{\bar{i}}$. Since we are identifying $\overline{\mathcal{Q}}_{+}$ with $\bar{Q}_{+}$of $S_{\text {pert }}=\int\left|d^{2} z\right|\left\{\bar{Q}_{+}, V\right\}$, we see that $V=D_{+} W=-i D_{+}\left(K_{i}(\Phi\right.$, $\left.\bar{\Phi}) \partial_{z} \Phi^{i}-\bar{K}_{\bar{i}}(\Phi, \bar{\Phi}) \partial_{z} \Phi^{\bar{i}}\right) / 2$. To compute $V$, note that since $D_{+} \bar{\Phi}=0$, we have $D_{+}\left(K_{i} \partial_{z} \Phi^{i}-\bar{K}_{\bar{i}} \partial_{z} \bar{\Phi}^{\bar{i}}\right)=K_{i, j} D_{+} \Phi^{j} \partial_{z} \Phi^{i}+K_{i} \partial_{z} D_{+} \Phi^{i}-\bar{K}_{\bar{i}, i} D_{+} \Phi^{i} \partial_{z}$ $\bar{\Phi}^{\bar{i}}$. By subtracting the total derivative $\partial_{z}\left(K_{i} D_{+} \Phi^{i}\right)$ which will not contribute to the action, we get $V=\frac{i}{2}\left(K_{i, \bar{j}}+\bar{K}_{\bar{j}, i}\right) \partial_{z} \bar{\Phi}^{\bar{j}} D_{+} \Phi^{i}-\frac{i}{2}\left(K_{i, j}-K_{j, i}\right)$ $D_{+} \Phi^{j} \partial_{z} \Phi^{i}$. To set $\bar{\theta}^{+}=\theta^{+}=0$ in $(3.26)$, we just need to set $\Phi^{i}=\phi^{i}$, $\bar{\Phi}^{\bar{i}}=\phi^{\bar{i}}$, and $D_{+} \Phi^{i}=\sqrt{2} \rho_{+}^{i}=2 i \psi_{\bar{z}}^{i}$, and let $\overline{\mathcal{Q}}_{+}$act as in (3.18). Hence, $V=$ $\left(-\left(\partial_{\bar{j}} K_{i}+\partial_{i} \bar{K}_{\bar{j}}\right) \psi_{\bar{z}}^{i} \partial_{z} \phi^{\bar{j}}+\left(\partial_{i} K_{j}-\partial_{j} K_{i}\right) \psi_{\bar{z}}^{i} \partial_{z} \phi^{j}\right)$. By setting the $\lambda_{z a} l_{\bar{z}}^{a}$ term in equation (2.13) to zero via the equation of motion $l_{\bar{z}}^{a}=0$, we can read off the hermitian metric $g_{i \bar{j}}$ used in Section 2.2 to construct the basic Lagrangian in $S_{\text {pert }}$, as well as the field called $T$ in Section 3.2. We have $g_{i \bar{j}}=\partial_{i} \bar{K}_{\bar{j}}+\partial_{\bar{j}} K_{i}$, as claimed in (3.22) above, and $T_{i j}=\left(\partial_{i} K_{j}-\partial_{j} K_{i}\right)$. From the last statement, and the definition $T=\frac{1}{2} T_{i j} d \phi^{i} \wedge d \phi^{j}$ in Section 3.2 , we see that $T=\partial K$ as a $(2,0)$-form. Thus, it follows from (3.23) that the curvature of the two-form field $T$ is $\mathcal{H}=d T=\bar{\partial} \partial K=2 i \partial \omega_{T}$. By virtue 
of $(3.24)$, the $(2,1)$-form $\mathcal{H}=2 i \partial \omega_{T}$ obeys $\partial \mathcal{H}=\bar{\partial} \mathcal{H}=0$. Hence, via the Cech-Dolbeault isomorphism, it can be interpreted as a class in $H^{1}\left(X, \Omega_{X}^{2, \text { cl }}\right)$.

As we will show via an example in Section $7.2, \mathcal{H}$ above will indeed play the same role as $\mathcal{H}$ in Section 3.2 as the moduli of the chiral algebra. However, while in the general analysis of Section $3.2, \mathcal{H}$ can be an arbitrary element of $H^{1}\left(X, \Omega_{X}^{2, c l}\right)$ of type $(3,0) \oplus(2,1)$, note that the analysis in this section tells us that if we are to consider unitary sigma models only, $\mathcal{H}$ must be restricted to just $(2,1)$-forms. It must also be expressible as $2 i \partial \omega_{T}$, i.e., $\omega_{T}$ defines the torsion $\mathcal{H}$ of $X$. From the last statement, we see that a nonvanishing $\mathcal{H}$ will mean that $\partial \omega_{T} \neq 0$. Thus, by turning on the moduli of the chiral algebra via a deformation $S_{T}$ of the action $S_{\text {pert }}$ by the three-form flux $\mathcal{H}$, one will effectively induce a non-Kähler deformation of the target space $X$.

\section{Anomalies of the twisted heterotic sigma model}

In this section, we will study the anomalies of the twisted heterotic sigma model. In essence, the model will fail to exist in the quantum theory if the anomaly conditions are not satisfied. We aim to determine what these conditions are. In this discussion, we shall omit the additional term $S_{T}$, as anomalies do not depend on continuously varying couplings such as this one.

To begin, let us first note from the action $S_{\text {pert }}$ in (2.15) that the kinetic energy term quadratic in the fermi fields $\psi^{i}$ and $\psi^{\bar{i}}$ is given by $(\psi, D \psi)=$ $\int\left|d^{2} z\right| g_{i \bar{j}} \psi^{i} D \psi^{\bar{j}}$, where $D$ is the $\partial$ operator on $\Sigma$ acting on sections $\Phi^{*}(\overline{T X})$, constructed using a pull-back of the Levi-Civita connection on $T X$. The other kinetic energy term quadratic in the fermi fields $\lambda_{a}$ and $\lambda^{a}$ is given by $(\lambda, \bar{D} \lambda)=\int\left|d^{2} z\right| \lambda_{a} \bar{D} \lambda^{a}$, where $\bar{D}$ is the $\bar{\partial}$ operator on $\Sigma$ acting on sections $\Phi^{*}(\mathcal{E})$, constructed using a pull-back of the gauge connection $A$ on $\mathcal{E}$. (Notice that we have omitted the $z$ and $\bar{z}$ indices of the fields, as they are irrelevant in the present discussion.) By picking a spin structure on $\Sigma$, one can equivalently interpret $D$ and $\bar{D}$ as the Dirac operator and its complex conjugate on $\Sigma$, acting on sections of $\mathcal{V}=\bar{K}^{-1 / 2} \otimes \Phi^{*}(\overline{T X})$ and $\mathcal{W}=K^{-1 / 2} \otimes \Phi^{*}(\mathcal{E})$, respectively, ${ }^{12}$ where $K$ is the canonical bundle of $\Sigma$ and $\bar{K}$ its complex conjugate.

\footnotetext{
${ }^{12}$ On a Kähler manifold such as $\Sigma$, the Dirac operator coincides with the Dolbeault operator $\partial+\partial^{\dagger}$ on $\bar{K}^{1 / 2}$. Since $\psi^{\bar{i}}$ is a zero-form on $\Sigma$, we have $\partial^{\dagger} \psi^{\bar{i}}=0$. Thus, $\partial+\partial^{\dagger}$ is effectively $\partial$ when acting on $\psi^{\bar{i}}$. Therefore, the action of $\partial$ on $\psi^{\bar{i}} \in \Gamma\left(\Phi^{*}(\overline{T X})\right)$ is equivalent to the action of the Dirac operator on sections of the bundle $\mathcal{V}=\bar{K}^{-1 / 2} \otimes$ $\Phi^{*}(\overline{T X})$. Similar arguments hold for the case of $\bar{D}, \lambda^{a}$ and the bundle $\mathcal{W}$.
} 
Next, note that the anomaly arises as an obstruction to defining the functional Grassmann integral of the action quadratic in the Fermi fields $\lambda_{a}, \lambda^{a}$, and $\psi^{i}, \psi^{\bar{i}}$, as a general function on the configuration space $\mathcal{C}$ of inequivalent connections [21]. Via the last paragraph, the Grassmann integral is given by the product of the determinant of $D$ with the determinant of $\bar{D}$. This can also be expressed as the determinant of $D+\bar{D}$. As argued in [21], one must think of the functional integral as a section of a complex determinant line bundle $\mathcal{L}$ over $\mathcal{C}$. Only if $\mathcal{L}$ is trivial would the integral be a global section and therefore a function on $\mathcal{C}$. Hence, the anomaly is due to the non-triviality of $\mathcal{L}$. The bundle $\mathcal{L}$ can be characterized completely by its restriction to a non-trivial two-cycle in $\mathcal{C}$ such as a two-sphere [22].

To be more precise, let us consider a family of maps $\Phi: \Sigma \rightarrow X$, parameterized by a two-sphere base which we will denote as $B$. In computing the path integral, we actually want to consider the universal family of all maps from $\Sigma$ to $X$. This can be represented by a single map $\hat{\Phi}: \Sigma \times B \rightarrow X$. The quantum path integral is anomaly-free if $\mathcal{L}$, as a complex line bundle over $B$, is trivial. Conversely, if $\mathcal{L}$ is trivial, it can be trivialized by a local Green-Schwarz anomaly-cancellation mechanism and the quantum theory will exist.

From the theory of determinant line bundles, we find that the basic obstruction to triviality of $\mathcal{L}$ is its first Chern class. By an application of the family index theorem to anomalies [23, 24], the first Chern class of $\mathcal{L}$ is given by $\pi\left(\operatorname{ch}_{2}(\mathcal{W})-\operatorname{ch}_{2}(\mathcal{V})\right)$, whereby $\pi: H^{4}(\Sigma \times B) \rightarrow H^{2}(B)$. Note that the anomaly lives in $H^{4}(\Sigma \times B)$ and not $H^{2}(B) ; \pi\left(\operatorname{ch}_{2}(\mathcal{W})-\operatorname{ch}_{2}(\mathcal{V})\right)$ vanishes if $\left(\operatorname{ch}_{2}(\mathcal{W})-\operatorname{ch}_{2}(\mathcal{V})\right)$ in $H^{4}(\Sigma \times B)$ vanishes before it is being mapped to $H^{2}(B)$. However, if $\left(\operatorname{ch}_{2}(\mathcal{W})-\operatorname{ch}_{2}(\mathcal{V})\right) \neq 0$ but $\pi\left(\operatorname{ch}_{2}(\mathcal{W})-\operatorname{ch}_{2}(\mathcal{V})\right)=0$, then even though $\mathcal{L}$ is trivial, it cannot be trivialized by a Green-Schwarz mechanism.

To evaluate the anomaly, first note that we have a Chern character identity $\operatorname{ch}(E \otimes F)=\operatorname{ch}(E) \operatorname{ch}(F)$, where $E$ and $F$ are any two bundles. Hence, by tensoring $\Phi^{*}(\mathcal{E})$ with $K^{-1 / 2}$ to obtain $\mathcal{W}$, we get an additional term $\frac{1}{2} c_{1}(\Sigma) c_{1}(\mathcal{E})$. Next, note that $\operatorname{ch}_{2}(\bar{E})=\operatorname{ch}_{2}(E)$, and by tensoring $\Phi^{*}(\overline{T X})$ with $\bar{K}^{-1 / 2}$ to obtain $\mathcal{V}$, we get an additional term $\frac{1}{2} c_{1}(\Sigma) c_{1}(T X)$. Therefore, the condition for vanishing anomaly will be given by

$$
0=\frac{1}{2} c_{1}(\Sigma)\left(c_{1}(\mathcal{E})-c_{1}(T X)\right)=\operatorname{ch}_{2}(\mathcal{E})-\operatorname{ch}_{2}(T X) .
$$

The first condition means that we can either restrict ourselves to Riemann surfaces $\Sigma$ with $c_{1}(\Sigma)=0$ and $\left(c_{1}(\mathcal{E})-c_{1}(T X)\right) \neq 0$ or allow $\Sigma$ to be arbitrary while $\left(c_{1}(\mathcal{E})-c_{1}(T X)\right)=0$. Notice also that the anomaly automatically vanishes if the bundles $T X$ and $\mathcal{E}$ are trivial such that $c_{n}(T X)=$ 
$c_{n}(\mathcal{E})=0$ for any $n \geq 1$, or if $\mathcal{E}=T X$. The latter condition will be important when we discuss what happens at the $(2,2)$ locus in Section 7 .

The characteristic class $\left(\operatorname{ch}_{2}(\mathcal{E})-\operatorname{ch}_{2}(T X)\right)$ corresponds to an element of the Cech cohomology group $H^{2}\left(X, \Omega_{X}^{2, \mathrm{cl}}\right) .{ }^{13}$ We will encounter it in this representation in Sections 5.5 and 5.6. Similarly, as explained in the footnote, $\left(c_{1}(\mathcal{E})-c_{1}(X)\right)$ corresponds to an element of $H^{1}\left(X, \Omega_{X}^{1, \text { cl }}\right)$, while $c_{1}(\Sigma)$ corresponds to a class in $H^{1}\left(\Sigma, \Omega_{X}^{1, \mathrm{cl}}\right)$. These will make a later appearance as well.

Note that the $\left(\operatorname{ch}_{2}(\mathcal{E})-\operatorname{ch}_{2}(T X)\right)$ anomaly appears in a heterotic sigma model with $(0,2)$ supersymmetry regardless of any topological twisting. The $\frac{1}{2} c_{1}(\Sigma)\left(c_{1}(\mathcal{E})-c_{1}(T X)\right)$ anomaly, however, only occurs in a heterotic $(0,2)$ theory that has been twisted.

\subsection{Additional observations}

Recall from Section 3.1 that the chiral algebra of local holomorphic operators requires a flat metric up to scaling on $\Sigma$ to be globally defined. Therefore, it can be defined over all $\Sigma$ for genus 1 . The obstruction to its global definition on $\Sigma$ of higher genera is captured by the $\frac{1}{2} c_{1}(\Sigma)\left(c_{1}(\mathcal{E})-c_{1}(T X)\right)$ anomaly. This can be seen as follows.

Note that, at this stage, we are considering the case where $\mathcal{E} \neq T X$. So, in general, $c_{1}(\mathcal{E}) \neq c_{1}(T X)$. In such an event, the anomaly depends solely on $c_{1}(\Sigma)$. If $c_{1}(\Sigma) \neq 0$, such as when $\Sigma$ is curved or of higher genera, the Ricci scalar $R$ of $\Sigma$ is non-vanishing. Thus, the expression of $T_{z \bar{z}}$ will be modified, such that

$$
T_{z \bar{z}}=\left\{\bar{Q}_{+}, G_{z \bar{z}}\right\}+\frac{c}{2 \pi} R,
$$

\footnotetext{
${ }^{13}$ As had been shown in [12], $\operatorname{ch}_{2}(T X)$ can be interpreted as an element of $H^{2}\left(X, \Omega_{X}^{2, \mathrm{cl}}\right)$, while $c_{k}(T X)$ can be interpreted as an element of $H^{1}\left(X, \Omega_{X}^{1, \mathrm{cl}}\right)$. Using similar arguments, we can also show that $\operatorname{ch}_{2}(\mathcal{E})$ corresponds to an element of $H^{2}\left(X, \Omega_{X}^{2, \text { cl }}\right)$ as follows. On any complex hermitian manifold, $\operatorname{ch}_{2}(\mathcal{E})$ can be represented by a closed form of type $(2,2)$. This can be seen by picking any connection on the holomorphic vector bundle $\mathcal{E}$ over $X$, whose $(0,1)$ part is the natural $\bar{\partial}$ operator of this bundle. Since $\bar{\partial}^{2}=0$, the curvature of such a connection is of type $(2,0) \oplus(1,1)$. However, as discussed in the footnote on p. 6 , the $(2,0)$ part of the curvature must vanish. Hence, the curvature is of type $(1,1)$. Therefore, for every $k \geq 0, c_{k}(\mathcal{E})$ is described by a closed form of type $(k, k)$. Thus, via the Cech-Dolbeault isomorphism, $c_{k}(\mathcal{E})$ represents an element of $H^{k}\left(X, \Omega_{X}^{k, \text { cl }}\right)$. In particular, $c_{1}(\mathcal{E})$ represents an element of $H^{1}\left(X, \Omega_{X}^{1, \mathrm{cl}}\right)$, and $\operatorname{ch}_{2}(\mathcal{E})=\frac{1}{2}\left(c_{1}^{2}(\mathcal{E})-2 c_{2}(\mathcal{E})\right)$ represents an element of $H^{2}\left(X, \Omega_{X}^{2, \mathrm{cl}}\right)$.
} 
where $c$ is a non-zero constant related to the central charge of the sigma model. The additional term on the RHS of (4.2), given by a multiple of $R$, represents a soft conformal anomaly on the worldsheet due to a curved $\Sigma$. $R$ scales as a $(1,1)$ operator as required.

There are consequences on the original nature of the $\bar{Q}_{+}$-cohomology of operators due to this additional term. Recall from Section 3.1 that the holomorphy of $T_{z z}$ holds so long as $\partial_{z} T_{z \bar{z}} \sim 0$. However, from the modified expression of $T_{z \bar{z}}$ in (4.2), we now find that $\partial_{z} T_{z \bar{z}} \nsim 0$. Hence, the invariance of the $\bar{Q}_{+}$-cohomology of operators under translations on the worldsheet, which requires $T_{z z}$ to be holomorphic in $z$, no longer holds. Therefore, the local holomorphic operators fail to define a chiral algebra that is globally valid over $\Sigma$, since one of the axioms of a chiral algebra is invariant under translations on the worldsheet.

On the other hand, the second term on the RHS of (4.2), being a $c$-number anomaly, will affect only the partition function and not the normalized correlation functions. Thus, as argued in Section 3.1, the correlation functions of local holomorphic operators will continue to depend on $\Sigma$ only via its complex structure (as is familiar for chiral algebras).

\section{Sheaf of perturbative observables}

\subsection{General considerations}

In general, a local operator is an operator $\mathcal{F}$ that is a function of the physical fields $\phi^{i}, \phi^{\bar{i}}, \psi_{\bar{z}}^{i}, \psi^{\bar{i}}, \lambda_{z a}, \lambda^{a}$ and their derivatives with respect to $z$ and $\bar{z}^{14,15}$ However, as we saw in Section 3.1, the $\bar{Q}_{+}$-cohomology vanishes for operators of dimension $(n, m)$ with $m \neq 0$. Since $\psi_{\bar{z}}^{i}$ and the derivative $\partial_{\bar{z}}$ both have $m=1$ (and recall from Section 3.1 that a physical operator cannot have negative $m$ or $n), \bar{Q}_{+}$-cohomology classes can be constructed from just $\phi^{i}, \phi^{\bar{i}}, \psi^{\bar{i}}, \lambda_{z a}, \lambda^{a}$ and their derivatives with respect to $z$. Note that the equation of motion for $\psi^{\bar{i}}$ is $D_{z} \psi^{\bar{i}}=-F^{a}{ }_{b}{ }^{\bar{j}} \bar{j}(\phi) \lambda_{z a} \lambda^{b} \psi^{\bar{j}}$. Thus, we can ignore the $z$-derivatives of $\psi^{\bar{i}}$, since it can be expressed in terms of the other fields and their corresponding derivatives. Therefore, a chiral (i.e., $\bar{Q}_{+}$-invariant)

\footnotetext{
${ }^{14}$ Notice that we have excluded the auxiliary fields $l_{z a}$ and $l_{\bar{z}}^{a}$, as they do not contribute to the correlation functions since their propagators are trivial.

${ }^{15}$ Note here that since we are interested in local operators which define a holomorphic chiral algebra on the Riemann surface $\Sigma$, we will work locally on a flat $\Sigma$ with local parameter $z$. Hence, we need not include in our operators the dependence on the scalar curvature of $\Sigma$.
} 
operator which represents a $\bar{Q}_{+}$-cohomology class is given by

$$
\begin{gathered}
\mathcal{F}\left(\phi^{i}, \partial_{z} \phi^{i}, \partial_{z}^{2} \phi^{i}, \ldots ; \phi^{\bar{i}}, \partial_{z} \phi^{\bar{i}}, \partial_{z}^{2} \phi^{\bar{i}}, \ldots ; \lambda_{z a}, \partial_{z} \lambda_{z a}, \partial_{z}^{2} \lambda_{z a},\right. \\
\left.\ldots ; \lambda^{a}, \partial_{z} \lambda^{a}, \partial_{z}^{2} \lambda^{a}, \ldots ; \psi^{\bar{i}}\right)
\end{gathered}
$$

where we have tried to indicate that $\mathcal{F}$ might depend on $z$ derivatives of $\phi^{i}, \phi^{\bar{i}}, \lambda_{z a}$, and $\lambda^{a}$ of arbitrarily high order, though not on derivatives of $\psi^{\bar{i}}$. If the scaling dimension of $\mathcal{F}$ is bounded, it will mean that $\mathcal{F}$ depends only on the derivatives of fields up to some finite order, is a polynomial of bounded degree in those, and/or is a bounded polynomial in $\lambda_{z a}$. Notice that $\mathcal{F}$ will always be a polynomial of finite degree in $\lambda^{a}, \lambda_{z a}$, and $\psi^{\bar{i}}$, simply because $\lambda^{a}, \lambda_{z a}$, and $\psi^{\bar{i}}$ are fermionic and can only have a finite number of components before they vanish due to their anti-commutativity. However, the dependence of $\mathcal{F}$ on $\phi^{i}, \phi^{\bar{i}}$ (as opposed to their derivatives) need not have any simple form. Nevertheless, we can make the following observation: from the $U(1)_{\mathrm{L}} \times U(1)_{\mathrm{R}}$ charges of the fields listed in Section 2.2, we see that if $\mathcal{F}$ is homogeneous of degree $k$ in $\psi^{\bar{i}}$, then it has $U(1)_{\mathrm{L}} \times U(1)_{\mathrm{R}}$-charge $\left(q_{\mathrm{L}}, q_{\mathrm{R}}\right)=(p, k)$, where $p$ is determined by the net number of $\lambda_{z a}$ over $\lambda^{a}$ fields (and/or of their corresponding derivatives) in $\mathcal{F}$.

A general $q_{\mathrm{R}}=k$ operator $\mathcal{F}\left(\phi^{i}, \partial_{z} \phi^{i}, \ldots ; \phi^{\bar{i}}, \partial_{z} \phi^{\bar{i}}, \ldots ; \lambda_{z a}, \partial_{z} \lambda_{z a}, \ldots ; \lambda^{a}\right.$, $\left.\partial_{z} \lambda^{a}, \ldots ; \psi^{\bar{i}}\right)$ can be interpreted as a $(0, k)$-form on $X$ with values in a certain tensor product bundle. In order to illustrate the general idea behind this interpretation, we will make things explicit for operators of dimension $(0,0)$ and $(1,0)$. Similar arguments will likewise apply for operators of higher dimension. For dimension $(0,0)$, the most general operator takes the form $\mathcal{F}\left(\phi^{i}, \phi^{\bar{i}} ; \lambda^{a} ; \psi^{\bar{j}}\right)=f_{\bar{j}_{1}, \ldots, \bar{j}_{k} ; a_{1}, \ldots, a_{q}}\left(\phi^{i}, \phi^{\bar{i}}\right) \psi^{\bar{j}_{i}} \ldots \psi^{\bar{j}_{k}} \lambda^{a_{1}} \ldots \lambda^{a_{q}}$; thus, $\mathcal{F}$ may depend on $\phi^{i}, \phi^{\bar{i}}$, and $\lambda^{a}$, but not on their derivatives, and is $k$ th order in $\psi^{\bar{j}}$. Mapping $\psi^{\vec{j}}$ to $d \phi^{\bar{j}}$, such an operator corresponds to an ordinary $(0, k)$-form $f_{\bar{j}_{1}, \ldots, \bar{j}_{k}}\left(\phi^{i}, \phi^{\bar{i}}\right) d \phi^{\bar{j}_{1}} \cdots d \phi^{\bar{j}_{k}}$ on $X$ with values in the bundle $\Lambda^{q} \mathcal{E}^{*}{ }^{16}$ For dimension $(1,0)$, there are four general cases. In the first case, we have an operator $\mathcal{F}\left(\phi^{l}, \partial_{z} \phi^{i}, \phi^{\bar{l}} ; \lambda^{a} ; \psi^{\bar{j}}\right)=f_{i, \bar{j}_{1}, \ldots, \bar{j}_{k} ; a_{1}, \ldots, a_{q}}\left(\phi^{l}, \phi^{\bar{l}}\right) \partial_{z} \phi^{i} \psi^{\bar{j}_{1}}$ $\ldots \psi^{\bar{j}_{k}} \lambda^{a_{1}} \ldots \lambda^{a_{q}}$ that is linear in $\partial_{z} \phi^{i}$ and does not depend on any other derivatives. It is a $(0, k)$-form on $X$ with values in the tensor product bundle of $T^{*} X$ with $\Lambda^{q} \mathcal{E}^{*}$; alternatively, it is a $(1, k)$-form on $X$ with values in the bundle $\Lambda^{q} \mathcal{E}^{*}$. Similarly, in the second case, we have an opera-

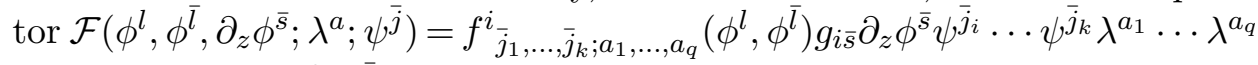
that is linear in $\partial_{z} \phi^{\bar{s}}$ and does not depend on any other derivatives. It is a $(0, k)$-form on $X$ with values in the tensor product bundle of $T X$ with $\Lambda^{q} \mathcal{E}^{*}$. In the third case, we have an operator $\mathcal{F}\left(\phi^{l}, \phi^{\bar{l}} ; \lambda^{a}, \partial_{z} \lambda^{a} ; \psi^{\bar{j}}\right)=$

\footnotetext{
${ }^{16}$ Note that $q \leq \operatorname{rank}(\mathcal{E})$ due to the anti-commutativity of $\lambda^{a}$.
} 
$f_{\bar{j}_{1}, \ldots, \bar{j}_{k} ; a_{1}, \ldots, a_{q}}^{\bar{b}}\left(\phi^{l}, \phi^{\bar{l}}\right) h_{\bar{b} a} \partial_{z} \lambda^{a} \psi^{\bar{j}_{1}} \cdots \psi^{\bar{j}_{k}} \lambda^{a_{1}} \cdots \lambda^{a_{q}}$ that is linear in $\partial_{z} \lambda^{a}$ and does not depend on any other derivatives. Such an operator corresponds to a $(0, k)$-form on $X$ with values in the (anti-symmetric) tensor product bundle of $\bar{E}$ with $\Lambda^{q} \mathcal{E}^{*}$, where the local holomorphic sections of the bundle $E$ are spanned by $\partial_{z} \lambda^{a}$. In the last case, we have an operator $\mathcal{F}\left(\phi^{l}, \phi^{\bar{l}} ; \lambda_{z a}, \lambda^{a} ; \psi^{\bar{j}}\right)=f_{\bar{j}_{1}, \ldots, \bar{j}_{k} ; a_{1}, \ldots, a_{q}}\left(\phi^{l}, \phi^{\bar{l}}\right) \lambda_{z a} \psi^{\bar{j}_{i}} \cdots \psi^{\bar{j}_{k}} \lambda^{a_{1}} \cdots \lambda^{a_{q}} ;$ here, $\mathcal{F}$ may depend on $\phi^{i}, \phi^{\bar{i}}, \lambda_{z a}$, and $\lambda^{a}$, but not on their derivatives. Such an operator corresponds to a $(0, k)$-form on $X$ with values in the (antisymmetric) tensor product bundle of $\mathcal{E}$ with $\Lambda^{q} \mathcal{E}^{*}$. In a similar fashion, for any integer $n>0$, the operators of dimension $(n, 0)$ and charge $q_{\mathrm{R}}=k$ can be interpreted as $(0, k)$-forms with values in a certain tensor product bundle over $X$. This structure persists in quantum perturbation theory, but there may be perturbative corrections to the complex structure of the bundle.

The action of $\bar{Q}_{+}$on such operators can be easily described at the classical level. If we interpret $\psi^{\bar{i}}$ as $d \phi^{\bar{i}}$, then $\bar{Q}_{+}$acts on functions of $\phi^{i}$ and $\phi^{\bar{i}}$ and is simply the $\bar{\partial}$ operator on $X$. This follows from the transformation laws $\delta \phi^{\bar{i}}=\psi^{\bar{i}}, \delta \phi^{i}=0, \delta \psi^{\bar{i}}=0$, and $\delta \lambda_{z a}=\delta \lambda^{a}=0$ (on-shell). Note that if the holomorphic vector bundle $\mathcal{E}$ has vanishing curvature, the interpretation of $\bar{Q}_{+}$as the $\bar{\partial}$ operator will remain valid when $\bar{Q}_{+}$acts on a more general operator $\mathcal{F}\left(\phi^{i}, \partial_{z} \phi^{i}, \ldots ; \phi^{\bar{i}}, \partial_{z} \phi^{\bar{i}}, \ldots ; \lambda_{z a}, \ldots ; \lambda^{a}, \ldots ; \psi^{\bar{i}}\right)$ that does depend on the derivatives of $\phi^{i}$ and $\phi^{i}$. The reason for this is that if $\mathcal{E}$ is a trivial bundle with zero curvature, we will have the equation of motion $D_{z} \psi^{\bar{i}}=0$. This means that one can neglect the action of $\bar{Q}_{+}$on derivatives $\partial_{z}^{m} \phi^{\bar{i}}$ with $m>0$. On the other hand, if $\mathcal{E}$ is a non-trivial holomorphic vector bundle, $\bar{Q}_{+}$will only act as the $\bar{\partial}$ operator on physical operators that do not contain the derivatives $\partial_{z}^{m} \phi^{\bar{i}}$ with $m>0$.

Perturbatively, there will be corrections to the action of $\bar{Q}_{+}$. In fact, as briefly mentioned in Section 3.1 earlier, (3.6) provides such an example - the holomorphic stress tensor $T_{z z}$, though not corrected at 1-loop, is no longer $\bar{Q}_{+}$-closed because the action of $\bar{Q}_{+}$has received perturbative corrections. Let us now attempt to better understand the nature of such perturbative corrections. To this end, let $Q_{\mathrm{cl}}$ denote the classical approximation to $\bar{Q}_{+}$. The perturbative corrections in $\bar{Q}_{+}$will then modify the classical expression $Q_{\mathrm{cl}}$. Note that since the sigma model perturbation theory is local on $X$, and it depends on an expansion of fields such as the metric tensor of $X$ in a Taylor series up to some given order, the perturbative corrections to $Q_{\mathrm{cl}}$ will also be local on $X$, where order by order, they consist of differential operators whose possible degree grows with the order of the perturbation theory. 
Let us now perturb the classical expression $Q_{\mathrm{cl}}$ so that $\bar{Q}_{+}=Q_{\mathrm{cl}}+\epsilon Q^{\prime}+$ $O\left(\epsilon^{2}\right)$, where $\epsilon$ is a parameter that controls the magnitude of the perturbative quantum corrections at each order of the expansion. To ensure that we continue to have $\bar{Q}_{+}^{2}=0$, we require that $\left\{Q_{\mathrm{cl}}, Q^{\prime}\right\}=0$. In addition, if $Q^{\prime}=\left\{Q_{\mathrm{cl}}, \Lambda\right\}$ for some $\Lambda$, then via the conjugation of $\bar{Q}_{+}$with $\exp (-\epsilon \Lambda)$ (which results in a trivial change of basis in the space of $\bar{Q}_{+}$-closed local operators), the correction by $Q^{\prime}$ can be removed. Hence, $Q^{\prime}$ represents a $Q_{\mathrm{cl}}$-cohomology class. Since $Q^{\prime}$ is to be generated in the sigma model perturbation theory, it must be constructed locally from the fields appearing in the sigma model action.

It will be useful for later if we discuss the case when $\mathcal{E}$ is a trivial bundle now. In such a case, $Q_{\mathrm{cl}}$ will always act as the $\bar{\partial}$ operator, as argued above. In other words, perturbative corrections to $\bar{Q}_{+}$will come from representatives of $\bar{\partial}$-cohomology classes on $X$. An example would be the Ricci tensor in (3.6) which represents a $\bar{\partial}$-cohomology class in $H^{1}\left(X, T^{*} X\right)$. It is also constructed locally from the metric of $X$, which appears in the action. Hence, it satisfies the conditions required of a perturbative correction $Q^{\prime}$. Another representative of a $\bar{\partial}$-cohomology class on $X$, which may contribute as a perturbative correction to the classical expression $\bar{Q}_{+}=Q_{\mathrm{cl}}$, would be an element of $H^{1}\left(X, \Omega_{X}^{2, c l}\right)$. It is also constructed locally from fields appearing in the action $S_{\text {pert }}$ and is used to deform the action. In fact, its interpretation as a perturbative correction $Q^{\prime}$ is consistent with its interpretation as the moduli of the chiral algebra. To see this, notice that its interpretation as $Q^{\prime}$ means that it will parameterize a family of $\bar{Q}_{+}=Q_{\mathrm{cl}}+\epsilon Q^{\prime}$ operators at the quantum level. Since the chiral algebra of local operators is defined to be closed with respect to the $\bar{Q}_{+}$operator, it will vary with the $\bar{Q}_{+}$operator and consequently with $H^{1}\left(X, \Omega_{X}^{2, \text { cl }}\right)$, i.e., one can associate the moduli of the chiral algebra with $H^{1}\left(X, \Omega_{X}^{2, \mathrm{cl}}\right)$. Apparently, these classes are the only one-dimensional $\bar{\partial}$-cohomology classes on $X$ that can be constructed locally from fields appearing in the action, and it may be that they completely determine the perturbative corrections to $\bar{Q}_{+}=Q_{\mathrm{cl}} \cdot{ }^{17}$ The observations in this paragraph will be important in Section 5.4, when we discuss the $\bar{Q}_{+^{-}}$ cohomology of local operators (on a small open set $U \subset X$ ) furnished by a sheaf of vertex superalgebras associated with a free $b c-\beta \gamma$ system.

\footnotetext{
${ }^{17}$ Since we are considering a holomorphic vector bundle $\mathcal{E}$ whose curvature two-form vanishes in this case, the second term of $\Delta_{1 \text {-loop }}$ in (3.5) will be zero. Consequently, only the first term on the RHS of (3.5) remains. In other words, only $R_{i \bar{j}}$ will contribute to the correction of $Q_{\mathrm{cl}}$ from $\Delta_{1-\text { loop }}$. Since an element of $H^{1}\left(X, \Omega_{X}^{2, \text { cl }}\right)$ is the only other $\bar{\partial}$-cohomology class which can appear in the quantum action, it would contribute as the only other perturbative correction to $Q_{\mathrm{cl}}$.
} 
The fact that $\bar{Q}_{+}$does not always act as the $\bar{\partial}$ operator even at the classical level seems to suggest that one needs a more general framework than just ordinary Dolbeault or $\bar{\partial}$-cohomology to describe the $\bar{Q}_{+}$-cohomology of the twisted heterotic sigma model. Indeed, as we will show shortly in Section 5.3 , the appropriate description of the $\bar{Q}_{+}$-cohomology of local operators spanning the chiral algebra will be given in terms of the more abstract notion of Cech cohomology.

\subsection{A topological chiral ring}

Next, let us make an interesting and relevant observation about the ground operators in the $\bar{Q}_{+}$-cohomology. Note that we had already shown in Section 3.1 that the $\bar{Q}_{+}$-cohomology of operators has the structure of a chiral algebra with holomorphic OPEs. Let the local operators of the $\bar{Q}_{+}$-cohomology be given by $\mathcal{F}_{a}, \mathcal{F}_{b}, \ldots$ with scaling dimensions $\left(h_{a}, 0\right),\left(h_{b}, 0\right), \ldots$ By holomorphy, and the conservation of scaling dimensions and $U(1)_{\mathrm{L}} \times U(1)_{\mathrm{R}}$ charges, the OPE of these operators takes the form

$$
\mathcal{F}_{a}(z) \mathcal{F}_{b}\left(z^{\prime}\right)=\sum_{q_{c}=q_{a}+q_{b}} \frac{C_{a b c} \mathcal{F}_{c}\left(z^{\prime}\right)}{\left(z-z^{\prime}\right)^{h_{a}+h_{b}-h_{c}}},
$$

where we have represented the $U(1)_{\mathrm{L}} \times U(1)_{\mathrm{R}}$ charges $\left(q_{\mathrm{L}}, q_{\mathrm{R}}\right)$ of the operators $\mathcal{F}_{a}, \mathcal{F}_{b}$, and $\mathcal{F}_{c}$ by $q_{a}, q_{b}$, and $q_{c}$ for brevity of notation. Here, $C_{a b c}$ is a structure constant that is (anti)symmetric in the indices. If $\widetilde{\mathcal{F}}_{a}$ and $\widetilde{\mathcal{F}}_{b}$ are ground operators of dimension $(0,0)$, i.e., $h_{a}=h_{b}=0$, the OPE will then be given by

$$
\widetilde{\mathcal{F}}_{a}(z) \widetilde{\mathcal{F}}_{b}\left(z^{\prime}\right)=\sum_{q_{c}=q_{a}+q_{b}} \frac{C_{a b c} \mathcal{F}_{c}\left(z^{\prime}\right)}{\left(z-z^{\prime}\right)^{-h_{c}}} .
$$

Notice that the RHS of (5.3) is only singular if $h_{c}<0$. Also recall that all physical operators in the $\bar{Q}_{+}$-cohomology cannot have negative scaling dimension, i.e., $h_{c} \geq 0 .{ }^{18}$ Hence, the RHS of (5.3), given by ( $z-$ $\left.z^{\prime}\right)^{h_{c}} \mathcal{F}_{c}\left(z^{\prime}\right)$, is non-singular as $z \rightarrow z^{\prime}$, since a pole does not exist. Note that $\left(z-z^{\prime}\right)^{h_{c}} \mathcal{F}_{c}\left(z^{\prime}\right)$ must also be annihilated by $\bar{Q}_{+}$and be in its cohomology, since $\widetilde{\mathcal{F}}_{a}$ and $\widetilde{\mathcal{F}}_{b}$ are. In other words, we can write $\widetilde{\mathcal{F}}_{c}\left(z, z^{\prime}\right)=(z-$

\footnotetext{
${ }^{18}$ As mentioned in the footnote of p. 10, for an operator of classical dimension $(n, m)$, anomalous dimensions due to RG flow may shift the values of $n$ and $m$ in the quantum theory. However, the spin $n-m$ remains unchanged. Hence, since the operators in the $\bar{Q}_{+}$-cohomology of the quantum theory will continue to have $m=0$ (due to a $\bar{Q}_{+}$-trivial anti-holomorphic stress tensor $T_{\bar{z} \bar{z}}$ at the quantum level), the value of $n$ is unchanged as we go from the classical to the quantum theory, i.e., $n \geq 0$ holds even at the quantum level.
} 
$\left.z^{\prime}\right)^{h_{c}} \mathcal{F}_{c}\left(z^{\prime}\right)$, where $\widetilde{\mathcal{F}}_{c}\left(z, z^{\prime}\right)$ is a dimension $(0,0)$ operator that represents a $\bar{Q}_{+}$-cohomology class. Thus, we can express the OPE of the ground operators as

$$
\widetilde{\mathcal{F}}_{a}(z) \widetilde{\mathcal{F}}_{b}\left(z^{\prime}\right)=\sum_{q_{c}=q_{a}+q_{b}} C_{a b c} \widetilde{\mathcal{F}}_{c}\left(z, z^{\prime}\right) .
$$

Since the only holomorphic functions without a pole on a Riemann surface are constants, it will mean that the operators $\widetilde{\mathcal{F}}$ are independent of the coordinate " $z$ " on $\Sigma$. Hence, they are completely independent of their insertion points and the metric on $\Sigma$. Therefore, we conclude that the ground operators of the $\bar{Q}_{+}$-cohomology define a topological chiral ring via their OPE

$$
\widetilde{\mathcal{F}}_{a} \widetilde{\mathcal{F}}_{b}=\sum_{q_{c}=q_{a}+q_{b}} C_{a b c} \widetilde{\mathcal{F}}_{c}
$$

In perturbation theory, the chiral ring will have a $\mathbb{Z} \times \mathbb{Z}$ grading by the $U(1)_{\mathrm{L}} \times U(1)_{\mathrm{R}}$ charges of the operators. However, since each charged, anticommuting, fermionic field cannot appear twice in the same operator, each operator will consist of only a finite number of them. Consequently, the individual $\mathbb{Z}$ grading will be reduced $\bmod 2$ to $\mathbb{Z}_{2}$, such that the ring is effectively $\mathbb{Z}_{2} \times \mathbb{Z}_{2}$ graded. Non-perturbatively, due to worldsheet instantons, the continuous $U(1)_{\mathrm{L}} \times U(1)_{\mathrm{R}}$ symmetry is reduced to a discrete subgroup. In order for this discrete symmetry to be non-anomalous, the values of the corresponding $U(1)_{\mathrm{L}} \times U(1)_{\mathrm{R}}$ charges can only be fractional multiples of $\pi$. More precisely, from the relevant index theorems, we find that the initial $\mathbb{Z} \times \mathbb{Z}$ grading by the $U(1)_{\mathrm{L}} \times U(1)_{\mathrm{R}}$ charges will be reduced to $\mathbb{Z}_{2 p} \times \mathbb{Z}_{2 k}$ by worldsheet instantons, where $2 p$ and $2 k$ are the greatest divisors of $c_{1}(\mathcal{E})$ and $c_{1}(T X)$, respectively.

At the classical level (i.e., in the absence of perturbative corrections), $\bar{Q}_{+}=Q_{\mathrm{cl}}$ will act on a dimension $(0,0)$ operator (i.e., one that does not contain the derivatives $\partial_{z}^{m} \phi^{\bar{i}}$ with $\left.m>0\right)$ as the $\bar{\partial}$ operator. Moreover, recall that any dimension $(0,0)$ operator $\widetilde{\mathcal{F}}_{d}$ with $\left(q_{\mathrm{L}}, q_{\mathrm{R}}\right)=(-q, k)$ will correspond to an ordinary $(0, k)$-form $f_{\bar{j}_{1}, \ldots, \bar{j}_{k}}\left(\phi^{i}, \phi^{\bar{i}}\right) d \phi^{\bar{j}_{1}} \wedge \cdots \wedge d \phi^{\bar{j}_{k}}$ on $X$ with values in the bundle $\Lambda^{q} \mathcal{E}^{\vee}$, where $\mathcal{E}^{\vee}$ is the dual of the bundle $\mathcal{E}$. Hence, via the Cech-Dolbeault isomorphism in ordinary differential geometry, the classical ring is just the graded Cech cohomology ring $H^{*}\left(X, \Lambda^{*} \mathcal{E}^{\vee}\right)$. In any case, the operators will either be non-Grassmannian or Grassmannian, obeying either commutators or anti-commutators, depending on whether they contain an even or odd number of fermionic fields. 


\subsection{A sheaf of chiral algebras}

We shall now explain the idea of a "sheaf of chiral algebras" on $X$. To this end, note that both the $\bar{Q}_{+}$-cohomology of local operators (i.e., operators that are local on the Riemann surface $\Sigma$ ) and the fermionic symmetry generator $\bar{Q}_{+}$can be described locally on $X$. Hence, one is free to restrict the local operators to be well defined not throughout $X$, but only on a given open set $U \subset X$. Since in perturbation theory, we are considering trivial maps $\Phi: \Sigma \rightarrow X$ with no multiplicities, an operator defined in an open set $U$ will have a sensible OPE with another operator defined in $U$. From here, one can naturally proceed to restrict the definition of the operators to smaller open sets, such that a global definition of the operators can be obtained by gluing together the open sets on their unions and intersections. From this description, in which one associates a chiral algebra, its OPEs, and chiral ring to every open set $U \subset X$, we get what is known mathematically as a "sheaf of chiral algebras". We shall call this sheaf $\widehat{\mathcal{A}}$.

\subsubsection{Description of $\mathcal{A}$ via Cech cohomology}

In perturbation theory, one can also describe the $\bar{Q}_{+}$-cohomology classes by a form of Cech cohomology. This alternative description will take us to the mathematical point of view on the subject $[1,3,5]$. In essence, we will show that the chiral algebra $\mathcal{A}$ of the $\bar{Q}_{+}$-cohomology classes of the twisted heterotic sigma model on a holomorphic vector bundle $\mathcal{E}$ over $X$ can be represented, in perturbation theory, by the classes of the Cech cohomology of the sheaf $\widehat{\mathcal{A}}$ of locally defined chiral operators. To this end, we shall generalize the argument in Section 3.2, which provides a Cech cohomological description of a $\bar{\partial}$-cohomology, to demonstrate an isomorphism between the $\bar{Q}_{+}$-cohomology classes and the classes of the Cech cohomology of $\widehat{\mathcal{A}}$.

Let us start by considering an open set $U \subset X$ that is isomorphic to a contractible space such as an open ball in $\mathbb{C}^{n}$, where $n=\operatorname{dim}_{\mathbb{C}}(X)$. Because $U$ is a contractible space, any bundle over $U$ will be trivial. By applying this statement on the holomorphic vector bundle $\mathcal{E}$ over $U$, we find that the curvature of $\mathcal{E}$ vanishes. From the discussion in Section 5.1, we find that $\bar{Q}_{+}$ will then act as the $\bar{\partial}$ operator on any local operator $\mathcal{F}$ in $U$. In other words, $\mathcal{F}$ can be interpreted as a $\bar{\partial}$-closed $(0, k)$-form with values in a certain tensor product bundle $\widehat{F}$ over $U$. Thus, in the absence of perturbative corrections at the classical level, any operator $\mathcal{F}$ in the $\bar{Q}_{+}$-cohomology will be classes of $H_{\bar{\partial}}^{0, k}(U, \widehat{F})$ on $U$. As explained, $\widehat{F}$ will also be a trivial bundle over $U$, which means that $\widehat{F}$ will always possess a global section, i.e., it corresponds to a soft sheaf. Since the higher Cech cohomologies of a soft sheaf are 
trivial [25], we will have $H_{\text {Cech }}{ }^{k}(U, \widehat{F})=0$ for $k>0$. Mapping this back to the Dolbeault cohomology via the Cech-Dolbeault isomorphism, we find that $H_{\bar{\partial}}^{0, k}(U, \widehat{F})=0$ for $k>0$. Note that small quantum corrections in the perturbative limit can only annihilate cohomology classes and not create them. Hence, in perturbation theory, it follows that the local operators $\mathcal{F}$ with positive values of $q_{\mathrm{R}}$ must vanish in $\bar{Q}_{+}$-cohomology on $U$.

Now consider a good cover of $X$ by open sets $\left\{U_{a}\right\}$. Since the intersection of open sets $\left\{U_{a}\right\}$ also give open sets (isomorphic to open balls in $\mathbb{C}^{n}$ ), $\left\{U_{a}\right\}$ and all of their intersections have the same property as $U$ described above: $\bar{\partial}$-cohomology and hence $\bar{Q}_{+}$-cohomology vanishe for positive values of $q_{\mathrm{R}}$ on $\left\{U_{a}\right\}$ and their intersections.

Let the operator $\mathcal{F}_{1}$ on $X$ be a $\bar{Q}_{+}$-cohomology class with $q_{\mathrm{R}}=1$. It is here that we shall import the usual arguments relating a $\bar{\partial}$ and Cech cohomology to demonstrate an isomorphism between the $\bar{Q}_{+}$-cohomology and a Cech cohomology. When restricted to an open set $U_{a}$, the operator $\mathcal{F}_{1}$ must be trivial in $\bar{Q}_{+}$-cohomology, i.e., $\mathcal{F}_{1}=\left\{\bar{Q}_{+}, \mathcal{C}_{a}\right\}$, where $\mathcal{C}_{a}$ is an operator of $q_{\mathrm{R}}=0$ that is well defined in $U_{a}$.

Now, since $\bar{Q}_{+}$-cohomology classes such as $\mathcal{F}_{1}$ can be globally defined on $X$, we have $\mathcal{F}_{1}=\left\{\bar{Q}_{+}, \mathcal{C}_{a}\right\}=\left\{\bar{Q}_{+}, \mathcal{C}_{b}\right\}$ over the intersection $U_{a} \cap U_{b}$, so $\left\{\bar{Q}_{+}, \mathcal{C}_{a}-\mathcal{C}_{b}\right\}=0$. Let $\mathcal{C}_{a b}=\mathcal{C}_{a}-\mathcal{C}_{b}$. For each $a$ and $b, \mathcal{C}_{a b}$ is defined in $U_{a} \cap U_{b}$. Therefore, for all $a, b, c$, we have

$$
\mathcal{C}_{a b}=-\mathcal{C}_{b a}, \quad \mathcal{C}_{a b}+\mathcal{C}_{b c}+\mathcal{C}_{c a}=0
$$

Moreover, for $\left(q_{\mathrm{R}}=0\right)$ operators $\mathcal{K}_{a}$ and $\mathcal{K}_{b}$, whereby $\left\{\bar{Q}_{+}, \mathcal{K}_{a}\right\}=\left\{\bar{Q}_{+}, \mathcal{K}_{b}\right\}$ $=0$, we have an equivalence relation

$$
\mathcal{C}_{a b} \sim \mathcal{C}^{\prime}{ }_{a b}=\mathcal{C}_{a b}+\mathcal{K}_{a}-\mathcal{K}_{b}
$$

Note that the collection $\left\{C_{a b}\right\}$ are operators in the $\bar{Q}_{+}$-cohomology with well-defined OPEs, and whose dimension $(0,0)$ subset furnishes a topological chiral ring with $q_{\mathrm{R}}=0$.

Since the local operators with positive values of $q_{\mathrm{R}}$ vanish in $\bar{Q}_{+}$-cohomology on an arbitrary open set $U$, the sheaf $\widehat{\mathcal{A}}$ of the chiral algebra of operators has for its local sections the $\psi^{\bar{i}}$-independent (i.e., $q_{\mathrm{R}}=0$ ) operators $\widehat{\mathcal{F}}\left(\phi^{i}, \partial_{z} \phi^{i}, \ldots ; \phi^{\bar{i}}, \partial_{z} \phi^{\bar{i}}, \ldots ; \lambda_{z a}, \partial_{z} \lambda_{z a}, \ldots ; \lambda^{a}, \partial_{z} \lambda^{a}, \ldots\right)$ that are annihilated by $\bar{Q}_{+}$. Each $C_{a b}$ with $q_{\mathrm{R}}=0$ is thus a section of $\widehat{\mathcal{A}}$ over the intersection $U_{a} \cap U_{b}$. From (5.6) and (5.7), we find that the collection $\left\{C_{a b}\right\}$ defines the elements of the first Cech cohomology group $H_{\text {Cech }}^{1}(X, \widehat{\mathcal{A}})$. 
Next, note that the $\bar{Q}_{+}$-cohomology classes are defined as those operators which are $\bar{Q}_{+}$-closed, modulo those which can be globally written as $\left\{\bar{Q}_{+}, \ldots\right\}$ on $X$. In other words, $\mathcal{F}_{1}$ vanishes in $\bar{Q}_{+}$-cohomology if we can write it as $\mathcal{F}_{1}=\left\{\bar{Q}_{+}, \mathcal{C}_{a}\right\}=\left\{\bar{Q}_{+}, \mathcal{C}_{b}\right\}=\left\{\bar{Q}_{+}, \mathcal{C}\right\}$, i.e., $\mathcal{C}_{a}=\mathcal{C}_{b}$ and hence $\mathcal{C}_{a b}=0$. Therefore, a vanishing $\bar{Q}_{+}$-cohomology with $q_{\mathrm{R}}=1$ corresponds to a vanishing first Cech cohomology. Thus, we have obtained a map between the $\bar{Q}_{+}$-cohomology with $q_{\mathrm{R}}=1$ and a first Cech cohomology.

Similar to the case of relating a $\bar{\partial}$ and Cech cohomology, one can also run everything backwards and construct an inverse of this map. Suppose we are given a family $\left\{\mathcal{C}_{a b}\right\}$ of sections of $\widehat{\mathcal{A}}$ over the corresponding intersections $\left\{U_{a} \cap U_{b}\right\}$ and they obey (5.6) and (5.7) so that they define the elements of $H^{1}(X, \widehat{\mathcal{A}})$. We can then proceed as follows. Let the set $\left\{f_{a}\right\}$ be partition of unity subordinates to the open cover of $X$ provided by $\left\{U_{a}\right\}$. This means that the elements of $\left\{f_{a}\right\}$ are continuous functions on $X$, and they vanish outside the corresponding elements in $\left\{U_{a}\right\}$ while obeying $\sum_{a} f_{a}=1$. Let $\mathcal{F}_{1, a}$ be a chiral operator defined in $U_{a}$ by $\mathcal{F}_{1, a}=\sum_{c}\left[\bar{Q}_{+}, f_{c}\right] \mathcal{C}_{a c}{ }^{19} \quad \mathcal{F}_{1, a}$ is well defined throughout $U_{a}$, since in $U_{a}$, $\left[\bar{Q}_{+}, f_{c}\right]$ vanishes wherever $\mathcal{C}_{a c}$ is not defined. Clearly, $\mathcal{F}_{1, a}$ has $q_{\mathrm{R}}=1$, since $\mathcal{C}_{a c}$ has $q_{\mathrm{R}}=0$ and $\bar{Q}_{+}$has $q_{\mathrm{R}}=1$. Moreover, since $\mathcal{F}_{1, a}$ is a chiral operator defined in $U_{a}$, it will mean that $\left\{\bar{Q}_{+}, \mathcal{F}_{1, a}\right\}=0$ over $U_{a}$. For any $a$ and $b$, we have $\mathcal{F}_{1, a}-\mathcal{F}_{1, b}=\sum_{c}\left[\bar{Q}_{+}, f_{c}\right]\left(\mathcal{C}_{a c}-\mathcal{C}_{b c}\right)$. Using (5.6), this is $\sum_{c}\left[\bar{Q}_{+}, f_{c}\right] \mathcal{C}_{a b}=\left[\bar{Q}_{+}, \sum_{c} f_{c}\right] \mathcal{C}_{a b}$. This vanishes since $\sum_{c} f_{c}=1$. Hence, $\mathcal{F}_{1, a}=\mathcal{F}_{1, b}$ on $U_{a} \cap U_{b}$, for all $a$ and $b$. In other words, we have found a globally defined $q_{\mathrm{R}}=1$ operator $\mathcal{F}_{1}$ that obeys $\left\{\bar{Q}_{+}, \mathcal{F}_{1}\right\}=0$ on $X$. Notice that $\mathcal{F}_{1, a}$ and thus $\mathcal{F}_{1}$ is not defined to be of the form $\left\{\bar{Q}_{+}, \ldots\right\}$. Therefore, we have obtained a map from the Cech cohomology group $H^{1}(X, \widehat{\mathcal{A}})$ to the $\bar{Q}_{+}$-cohomology group with $q_{\mathrm{R}}=1$, i.e., $\bar{Q}_{+}$-closed $q_{\mathrm{R}}=1$ operators modulo those that can be globally written as $\left\{\bar{Q}_{+}, \ldots\right\}$. The fact that this map is an inverse of the first map can indeed be verified.

Since there is nothing unique about the $q_{\mathrm{R}}=1$ case, we can repeat the above procedure for operators with $q_{\mathrm{R}}>1$. In doing so, we find that the $\bar{Q}_{+}$-cohomology coincides with the Cech cohomology of $\widehat{\mathcal{A}}$ for all $q_{\mathrm{R}}$. Hence, the chiral algebra $\mathcal{A}$ of the twisted heterotic sigma model will be given by $\bigoplus_{q_{\mathrm{R}}} H_{\text {Cech }}^{q_{\mathrm{R}}}(X, \widehat{\mathcal{A}})$ as a vector space. As there will be no ambiguity, we shall henceforth omit the label "Cech" when referring to the cohomology of $\widehat{\mathcal{A}}$.

\footnotetext{
${ }^{19}$ Normal ordering of the operator product of $\left[\bar{Q}_{+}, f_{c}\left(\phi^{i}, \phi^{\bar{i}}\right)\right]$ with $\mathcal{C}_{a c}$ is needed for regularization purposes.
} 
Note that in the mathematical literature, the sheaf $\widehat{\mathcal{A}}$, also known as a sheaf of vertex superalgebras, is studied purely from the Cech viewpoint; the field $\psi^{\bar{i}}$ is omitted and locally on $X$, one considers operators constructed only from $\phi^{i}, \phi^{\bar{i}}, \lambda_{z a}, \lambda^{a}$ and their $z$-derivatives. The chiral algebra $\mathcal{A}$ of $\bar{Q}_{+}$-cohomology classes with positive $q_{\mathrm{R}}$ are correspondingly constructed as Cech $q_{\mathrm{R}}$-cocycles. However, in the physical description via a Lagrangian and $\bar{Q}_{+}$operator, the sheaf $\widehat{\mathcal{A}}$ and its cohomology are given a $\bar{\partial}$-like description, where Cech $q_{\mathrm{R}}$-cycles are represented by operators that are $q_{\mathrm{R}}$ th order in the field $\psi^{\bar{i}}$. Notice that the mathematical description does not involve any form of the perturbation theory at all. Instead, it utilizes the abstraction of Cech cohomology to define the spectrum of operators in the quantum sigma model. It is in this sense that the study of the sigma model is given a rigorous foundation in the mathematical literature.

\subsubsection{The constraint $\Lambda^{r} \mathcal{E}^{\vee} \cong K_{X}$}

In addition to the anomaly-cancellation conditions discussed in Section 4, there is another constraint that the sigma model must obey. This constraint has been discussed in [15], and we will repeat the discussion here for completeness. In a physical heterotic string compactification on a gauge bundle $\mathcal{E}$ over a space $X$, the charged massless $\mathrm{RR}$ states are represented (in the perturbative limit, ignoring worldsheet instantons) by classes in the Cech cohomology group [26]

$$
H^{q}\left(X, \Lambda^{p} \mathcal{E}^{\vee}\right)
$$

where $\mathcal{E}^{\vee}$ is the dual bundle of $\mathcal{E}$, and the corresponding vertex operators representing these states contain $p$ left-moving and $q$ right-moving fermi fields. Notice that the classes of (5.8) can also represent the dimension $(0,0)$ local operators of the $\bar{Q}_{+}$-cohomology in the twisted heterotic sigma model with $U(1)_{\mathrm{L}} \times U(1)_{\mathrm{R}}$ charge $(-p, q)$. It is here that the physical relevance of the sigma model is readily manifest.

In the context of the physical heterotic string with $(0,2)$ worldsheet supersymmetry, one can sometimes speak sensibly of a heterotic chiral ring. This ring is described additively by the sum of Cech cohomology groups of the form in (5.8), i.e.,

$$
H_{\mathrm{het}}^{*, *}=\sum_{p, q} H^{q}\left(X, \Lambda^{p} \mathcal{E}^{\vee}\right) .
$$

Note that the Serre duality in $(0,2)$ theories require that states in $H_{\text {het }}^{*, *}$ be dual to other states in $H_{\text {het }}^{*, *}$ [26]. Serre duality acts as

$$
\begin{aligned}
H^{i}\left(X, \Lambda^{j} \mathcal{E}^{\vee}\right) & \cong H^{n-i}\left(X, \Lambda^{j} \mathcal{E} \otimes K_{X}\right)^{*} \\
& \cong H^{n-i}\left(X, \Lambda^{r-j} \mathcal{E}^{\vee} \otimes \Lambda^{r} \mathcal{E} \otimes K_{X}\right)^{*},
\end{aligned}
$$


where $n=\operatorname{dim}_{\mathbb{C}} X$ and $r$ is the rank of $\mathcal{E} . K_{X}$ is simply the canonical bundle of $X$ (i.e., the bundle over $X$ whose holomorphic sections are $(n, 0)$ forms on $X)$. Hence, from (5.10), the states of $H_{\text {het }}^{*, *}$ only close back onto themselves under a duality relation if and only if the line bundle $\Lambda^{r} \mathcal{E} \otimes$ $K_{X}$ on $X$ is trivial, i.e., $\Lambda^{r} \mathcal{E}^{\vee} \cong K_{X}$. Thus, if the twisted heterotic sigma model is to be physically relevant such as to have a geometrical background that is consistent with one that will be considered in the actual, physical heterotic string theory, this constraint needs to be imposed. In fact, $\Lambda^{r} \mathcal{E}^{\vee} \cong$ $K_{X}$ implies $c_{1}(\mathcal{E})=c_{1}(T X)$. This condition on the first Chern class of the bundles is essential in a consistent definition of the twist, as previously discussed in Section 2.2.

\subsection{Relation to a free $b c-\beta \gamma$ system}

Now, we shall express in a physical language a few key points that are made in the mathematical literature $[3,5]$ starting from a Cech viewpoint. Let us start by providing a convenient description of the local structure of the sheaf $\widehat{\mathcal{A}}$. To this end, we will describe in a new way the $\bar{Q}_{+}$-cohomology of operators that are regular in a small open set $U \subset X$. We assume that $U$ is isomorphic to an open ball in $\mathbb{C}^{n}$ and is thus contractible.

Notice from $S_{\text {pert }}$ in (2.16) and $V$ in (2.13) that the hermitian metric on $X$ and the fibre metric of $\mathcal{E}$ (implicit in the second term $\lambda_{z a} l_{\bar{z}}^{a}$ of $V$ ) only appear inside a term of the form $\left\{\bar{Q}_{+}, \ldots\right\}$ in the action. Thus, any shift in the metrics will also appear inside $\bar{Q}_{+}$-exact (i.e., $\bar{Q}_{+}$-trivial) terms. Consequently, for our present purposes, we can arbitrarily redefine the values of the hermitian metric on $X$ and the fibre metric of $\mathcal{E}$, since they do not affect the analysis of the $\bar{Q}_{+}$-cohomology. Therefore, to describe the local structure, we can pick a hermitian metric that is flat when restricted to $U$. Similarly, we can pick a fibre metric of $\mathcal{E}$ that is flat over $U$ as well. In fact, this latter choice is automatically satisfied in $U$ - the bundle $\mathcal{E}$ over a contractible space $U$ is trivial. The action, in general, also contains terms derived from an element of $H^{1}\left(X, \Omega_{X}^{2, \text { cl }}\right)$, as we explained in Section 3.2. From (3.8), we see that these terms are also $\bar{Q}_{+}$-exact locally and therefore can be discarded in analysing the local structure in $U$. Thus, the local action (derived from the flat fibre and hermitian metric) of the twisted heterotic sigma model on $\mathcal{E}_{\mathrm{f}} \times U$ (where $\mathcal{E}_{\mathrm{f}}$ denotes the fibre space of $\mathcal{E}$ ) is

$$
I=\frac{1}{2 \pi} \int_{\Sigma}\left|d^{2} z\right| \sum_{i, \bar{j}} \delta_{i \bar{j}}\left(\partial_{z} \phi^{\bar{j}} \partial_{\bar{z}} \phi^{i}+\psi_{\bar{z}}^{i} \partial_{z} \psi^{\bar{j}}\right)+\sum_{a, \bar{b}} \delta_{a \bar{b}} \lambda_{z}^{\bar{b}} \partial_{\bar{z}} \lambda^{a}
$$


where $\lambda_{z}^{\bar{b}}$ is a $(1,0)$-form on $\Sigma$ with values in the pull-back bundle $\Phi^{*}(\overline{\mathcal{E}})$, such that for an arbitrary fibre metric $h_{a \bar{b}}$, we have $\lambda_{z a}=h_{a \bar{b}} \lambda_{z}^{\bar{b}}$.

Now let us describe the $\bar{Q}_{+}$-cohomology classes of operators regular in $U$. As explained earlier, these are operators of dimension $(n, 0)$ that are independent of $\psi^{\bar{i}}$. In general, such operators are of the form $\widehat{\mathcal{F}}\left(\phi^{i}, \partial_{z} \phi^{i}, \ldots ; \phi^{\bar{i}}, \partial_{z} \phi^{\bar{i}}\right.$, $\left.\ldots ; \lambda_{z a}, \partial_{z} \lambda_{z a}, \ldots ; \lambda^{a}, \partial_{z} \lambda^{a}, \ldots\right)$. Note that since $\mathcal{E}$ has vanishing curvature over $U$, from the discussion in Section 5.1 , we see that $\bar{Q}_{+}$will act as the $\bar{\partial}$ operator at the classical level. In this case, the $\bar{Q}_{+}$operator can receive perturbative corrections from $\bar{\partial}$-cohomology classes such as the Ricci tensor and classes in $H^{1}\left(X, \Omega_{X}^{2, c l}\right)$. However, note that since we have picked a flat hermitian metric on $U$, the corresponding Ricci tensor on $U$ is zero. Moreover, as explained above, classes from $H^{1}\left(X, \Omega_{X}^{2, \mathrm{cl}}\right)$ do not contribute when analysing the $\bar{Q}_{+}$-cohomology on $U$. Hence, we can ignore the perturbative corrections to $\bar{Q}_{+}$for our present purposes. Therefore, on the classes of operators in $U, \bar{Q}_{+}$acts as $\bar{\partial}=\psi^{\bar{i}} \partial / \partial \phi^{\bar{i}}$, and the condition that $\widehat{\mathcal{F}}$ is annihilated by $\bar{Q}_{+}$is precisely that, as a function of $\phi^{i}, \phi^{\bar{i}}, \lambda_{z a}, \lambda^{a}$, and their $z$-derivatives, it is independent of $\phi^{\bar{i}}$ (as opposed to its derivatives) and depends only on the other variables, namely $\phi^{i}, \lambda_{z a}, \lambda^{a}$, and the derivatives of $\phi^{i}, \phi^{\bar{i}}, \lambda_{z a}$, and $\lambda^{a}{ }^{20}$ Hence, the $\bar{Q}_{+}$-invariant operators are of the form $\widehat{\mathcal{F}}\left(\phi^{i}, \partial_{z} \phi^{i}, \ldots ; \partial_{z} \phi^{\bar{i}}, \partial_{z}^{2} \phi^{\bar{i}}, \ldots ; \lambda_{z a}, \partial_{z} \lambda_{z a}, \partial_{z}^{2} \lambda_{z a}, \ldots ; \lambda^{a}, \partial_{z} \lambda^{a}, \partial_{z}^{2} \lambda^{a}, \ldots\right)$. In other words, the operators, in their dependence on the centre of mass coordinate of the string whose worldsheet theory is the twisted heterotic sigma model, are holomorphic. The local sections of $\widehat{\mathcal{A}}$ are just given by the operators in the $\bar{Q}_{+}$-cohomology of the local, twisted heterotic sigma model with action (5.11).

Let us set $\beta_{i}=\delta_{i \bar{j}} \partial_{z} \phi^{\bar{j}}$ and $\gamma^{i}=\phi^{i}$, whereby $\beta_{i}$ and $\gamma^{i}$ are bosonic operators of dimension $(1,0)$ and $(0,0)$, respectively. Next, let us set $\delta_{a \bar{b}} \lambda_{z}^{\bar{b}}=b_{a}$ and $\lambda^{a}=c^{a}$, whereby $b_{a}$ and $c^{a}$ are fermionic operators of dimension $(1,0)$ and $(0,0)$ accordingly. Then, the $\bar{Q}_{+}$-cohomology of operators regular in $U$ can be represented by arbitrary local functions of $\beta, \gamma, b$, and $c$ of the form $\widehat{\mathcal{F}}\left(\gamma, \partial_{z} \gamma, \partial_{z}^{2} \gamma, \ldots, \beta, \partial_{z} \beta, \partial_{z}^{2} \beta, \ldots, b, \partial_{z} b, \partial_{z}^{2} b, \ldots, c, \partial_{z} c, \partial_{z}^{2} c, \ldots\right)$. The operators $\beta$ and $\gamma$ have the operator products of a standard $\beta \gamma$ system. The products $\beta \cdot \beta$ and $\gamma \cdot \gamma$ are non-singular, while

$$
\beta_{i}(z) \gamma^{j}\left(z^{\prime}\right)=-\frac{\delta_{i j}}{z-z^{\prime}}+\text { regular. }
$$

\footnotetext{
${ }^{20}$ We can again ignore the action of $\bar{Q}_{+}$on $z$-derivatives of $\phi^{\bar{i}}$ because of the equation of motion $\partial_{z} \psi^{\bar{i}}=0$ and the symmetry transformation law $\delta \phi^{\bar{i}}=\psi^{\bar{i}}$.
} 
Similarly, the operators $b$ and $c$ have the operator products of a standard $b c$ system. The products $b \cdot b$ and $c \cdot c$ are non-singular, while

$$
b_{a}(z) c^{b}\left(z^{\prime}\right)=\frac{\delta_{a b}}{z-z^{\prime}}+\text { regular. }
$$

These statements can be deduced from the flat action (5.11) by standard methods. We can write down an action for the fields $\beta, \gamma, b$, and $c$, regarded as free elementary fields, which reproduces these OPEs. It is simply the following action of a $b c-\beta \gamma$ system:

$$
I_{b c-\beta \gamma}=\frac{1}{2 \pi} \int\left|d^{2} z\right|\left(\sum_{i} \beta_{i} \partial_{\bar{z}} \gamma^{i}+\sum_{a} b_{a} \partial_{\bar{z}} c^{a}\right) .
$$

Hence, we find that the local $b c-\beta \gamma$ system above reproduces the $\bar{Q}_{+}$-cohomo logy of $\psi^{\bar{i}}$-independent operators of the sigma model on $U$, i.e., the local sections of the sheaf $\widehat{\mathcal{A}}$.

At this juncture, one can make another important observation concerning the relationship between the local twisted heterotic sigma model with action (5.11) and the local version of the $b c-\beta \gamma$ system of (5.14). To begin with, note that the holomorphic stress tensor $\widehat{T}(z)=-2 \pi T_{z z}$ of the local sigma model is given by

$$
\widehat{T}(z)=-\delta_{i \bar{j}} \partial_{z} \phi^{\bar{j}} \partial_{z} \phi^{i}-\delta_{a \bar{b}} \lambda_{z}^{\bar{b}} \partial_{z} \lambda^{a}
$$

(Here and below, normal ordering is understood for $\widehat{T}(z)$ ). Via the respective identification of the fields $\beta$ and $\gamma$ with $\partial_{z} \phi$ and $\phi, \lambda_{z a}$, and $\lambda^{a}$ with $b_{a}$ and $c^{a}$, we find that $\widehat{T}(z)$ can be written in terms of the $b$ and $c$ fields as

$$
\widehat{T}(z)=-\beta_{i} \partial_{z} \gamma^{i}-b_{a} \partial_{z} c^{a} .
$$

$\widehat{T}(z)$, as given by (5.16), coincides with the holomorphic stress tensor of the local $b c-\beta \gamma$ system. Simply put, the twisted heterotic sigma model and the $b c-\beta \gamma$ system have the same local holomorphic stress tensor. This means that locally on $X$ (and hence $\mathcal{E} \rightarrow X$ ), the sigma model and the $b c-\beta \gamma$ system have the same generators of general holomorphic coordinate transformations on the worldsheet.

One may now ask the following question: does the $b c-\beta \gamma$ system reproduce the $\bar{Q}_{+}$-cohomology of $\psi^{\bar{i}}$-independent operators globally on $X$ or only in a small open set $U$ ? Well, the $b c-\beta \gamma$ system will certainly reproduce the $\bar{Q}_{+}$-cohomology of $\psi^{\bar{i}}$-independent operators globally on $X$ if there is no obstruction to defining the system globally on $X$, i.e., one finds, after making global sense of action (5.14), that the corresponding theory remains anomaly-free. Let us look at this more closely. 
First and foremost, the classical action (5.14) makes sense globally if we interpret the bosonic fields $\beta, \gamma$, and the fermionic fields $b, c$, correctly. $\gamma$ defines a map $\gamma: \Sigma \rightarrow X$, and $\beta$ is a $(1,0)$-form on $\Sigma$ with values in the pullback $\gamma^{*}\left(T^{*} X\right)$. The field $c$ is a scalar on $\Sigma$ with values in the pull-back $\gamma^{*}(\mathcal{E})$, while the field $b$ is a $(1,0)$-form on $\Sigma$ with values in the pull-back $\gamma^{*}\left(\mathcal{E}^{*}\right)$. With this interpretation, (5.14) becomes the action of what one might call a non-linear $b c-\beta \gamma$ system. However, by choosing $\gamma^{i}$ to be local coordinates on a small open set $U \subset X$, and $c^{a}$ to be local sections of the pull-back $\gamma^{*}(\mathcal{E})$ over $U$, one can make the action linear. In other words, a local version of (5.14) represents the action of a linear $b c-\beta \gamma$ system. To the best of the author's knowledge, the non-linear $b c-\beta \gamma$ system with action (5.14) does not seem to have been studied anywhere in the physics literature. Nevertheless, the results derived in this paper will definitely serve to provide additional insights into future problems involving the application of this non-linear $b c-\beta \gamma$ system.

Now that we have made global sense of the action of the $b c-\beta \gamma$ system at the classical level, we move on to discuss what happens at the quantum level. The anomalies that enter in the twisted heterotic sigma model also appear in the nonlinear $b c-\beta \gamma$ system. Expand around a classical solution of the nonlinear $b c-\beta \gamma$ system, represented by a holomorphic map $\gamma_{0}: \Sigma \rightarrow X$, and a section $c_{0}$ of the pull-back $\gamma_{0}^{*}(\mathcal{E})$. Setting $\gamma=\gamma_{0}+\gamma^{\prime}$ and $c=c_{0}+c^{\prime}$, the action, expanded to quadratic order about this solution, is $(1 / 2 \pi)\left[\left(\beta, \bar{D} \gamma^{\prime}\right)+\left(b, \bar{D} c^{\prime}\right)\right] . \quad \gamma^{\prime}$, being a deformation of the coordinate $\gamma_{0}$ on $X$, is a section of the pull-back $\gamma_{0}^{*}(T X)$. Thus, the kinetic operator of the $\beta$ and $\gamma$ fields is the $\bar{D}$ operator on sections of $\gamma_{0}^{*}(T X)$; it is the complex conjugate of the $D$ operator whose anomalies we encountered in Section 4. Complex conjugation reverses the sign of the anomalies, but here the fields are bosonic, while in Section 4, they were fermionic; this gives a second sign change. (Notice that the $D$ operator in Section 4 acts on sections of the pull-back of the anti-holomorphic bundle $\overline{T X}$ instead of the holomorphic bundle $T X$. However, this difference is irrelevant with regard to anomalies since $\operatorname{ch}_{2}(\bar{E})=\operatorname{ch}_{2}(E)$ for any holomorphic vector bundle $E$.) Next, since $c^{\prime}$ is a deformation of $c_{0}$, it will be a section of the pull-back $\gamma_{0}^{*}(\mathcal{E})$. The kinetic operator of the $b$ and $c$ fields is therefore the $\bar{D}$ operator on sections of $\gamma_{0}^{*}(\mathcal{E})$. This is the same $\bar{D}$ operator whose anomalies we encountered in Section 4. Moreover, the $\bar{D}$ operator in Section 4 also acts on sections of the pull-back of the bundle $\mathcal{E}$. Hence, the non-linear $b c-\beta \gamma$ system has exactly the same anomalies as the underlying twisted heterotic sigma model. And if the anomalies vanish, the $b c$ $\beta \gamma$ system will reproduce the $\bar{Q}_{+}$-cohomology of $\psi^{\bar{i}}$-independent operators globally on $X$. In other words, one can find a global section of $\widehat{\mathcal{A}}$ in such a case. 
Via the identification of the various fields mentioned above, the left-moving fields $b_{a}$ and $c^{a}$ will have $U(1)_{\mathrm{L}}$ charges $q_{\mathrm{L}}=1$ and $q_{\mathrm{L}}=-1$, respectively. Notice that this $U(1)_{\mathrm{L}}$ symmetry is nothing but the usual $U(1) \mathrm{R}$ ghost number symmetry of action (5.14) with the correct charges. However, note that the $b c-\beta \gamma$ system lacks the presence of right-moving fermions and thus the $U(1)_{\mathrm{R}}$ charge $q_{\mathrm{R}}$ carried by the fields $\psi_{\bar{z}}^{i}$ and $\psi^{\bar{i}}$ of the underlying twisted heterotic sigma model. Locally, the $\bar{Q}_{+}$-cohomology of the sigma model is non-vanishing only for $q_{\mathrm{R}}=0$. Globally, however, there can generically be cohomology in higher degrees. Since the chiral algebra of operators furnished by the linear $b c-\beta \gamma$ system gives the correct description of the $\bar{Q}_{+}$-cohomology of $\psi^{\bar{i}}$-independent operators on $U$, one can then expect the globally defined chiral algebra of operators furnished by the non-linear $b c-\beta \gamma$ system to correctly describe the $\bar{Q}_{+}$-cohomology classes of zero degree (i.e., $q_{\mathrm{R}}=0$ ) on $X$. How then can one use the nonlinear $b c-\beta \gamma$ system to describe the higher cohomology? The answer lies in the analysis carried out in Section 5.3. In the $b c-\beta \gamma$ description, we do not have a close analogue of $\bar{\partial}$ cohomology at our convenience. Nevertheless, we can use the more abstract notion of Cech cohomology. As before, we begin with a good cover of $X$ by small open sets $\left\{U_{a}\right\}$, and, as explained in Section 5.3, we can then describe the $\bar{Q}_{+}$-cohomology classes of positive degree (i.e., $q_{\mathrm{R}}>0$ ) by Cech $q_{\mathrm{R}}$-cocycles, i.e., they can be described by the $q_{\mathrm{R}}$ th Cech cohomology of the sheaf $\widehat{\mathcal{A}}$ of the chiral algebra of the linear $b c-\beta \gamma$ system, with action being a linearized version of (5.14). Although unusual from a physicist's perspective, this Cech cohomology approach has been taken as a starting point for the present subject in the mathematical literature $[1-3,5]$. Other more algebraic approaches to the subject have also been taken in [6].

Another issue that remains to be elucidated is the appearance of the respective moduli of the sigma model in the non-linear $b c-\beta \gamma$ system. Recall from Section 3.2 that the moduli of the chiral algebra of the sigma model consist of the complex and holomorphic structure of $X$ and $\mathcal{E}$, respectively, as well as a class in $H^{1}\left(X, \Omega_{X}^{2, \mathrm{cl}}\right)$. The complex and holomorphic structures are built into the classical action (5.14) via the definition of the fields themselves. However, one cannot incorporate a class from $H^{1}\left(X, \Omega_{X}^{2, c l}\right)$ within the action in this framework. Nevertheless, as we will explain in Section 5.6 , the modulus represented by a class in $H^{1}\left(X, \Omega_{X}^{2, \text { cl }}\right)$ can be built into the definition of specific Cech cocycles through which one can define a family of sheaves of chiral algebras. This approach has also been taken in the mathematical literature $[3,5]$.

A final remark to be made is that in the study of quantum field theory, one would like to be able to do more than just define the $\bar{Q}_{+}$-cohomology classes 
or a sheaf of chiral algebras. One would also like to be able to compute physically meaningful quantities such as the correlation functions of these cohomology classes of local operators. In the sigma model, the correlation functions can be computed from standard methods in the quantum field theory. But at first sight, there seems to be an obstacle in doing likewise for the non-linear $b c-\beta \gamma$ system. This can be seen as follows. Let the correlation function of $s$ local operators $\mathcal{O}_{1}, \mathcal{O}_{2}, \ldots, \mathcal{O}_{s}$ on a genus $g$ Riemann surface $\Sigma$ be given by $\left\langle\mathcal{O}_{1}\left(z_{1}\right) \ldots \mathcal{O}_{s}\left(z_{s}\right)\right\rangle_{g}$, where $\mathcal{O}_{i}\left(z_{i}\right)$ has $U(1)_{\mathrm{R}}$ charge $q_{\mathrm{R}}=q_{i}$. Note that the $U(1)_{\mathrm{R}}$ anomaly computation of $(2.8)$ in Section 2.2 means that for the correlation functions of our model to be non-vanishing, they must satisfy $\sum_{i} q_{i}=n(1-g)$ in perturbation theory (in the absence of worldsheet instantons). Thus, generic non-zero correlation functions require that not all the $q_{i}$ 's be zero. In particular, correlation functions at string tree level vanish unless $\sum_{i} q_{i}=n$, where $n=\operatorname{dim}_{\mathbb{C}} X$. However, the operators of $q_{i} \neq 0$ cannot be represented in a standard way in the non-linear $b c-\beta \gamma$ system. They are instead described by Cech $q_{i}$-cocycles. This means that in order for one to compute the corresponding correlation functions using the non-linear $b c-\beta \gamma$ system, one must translate the usual quantum field theory recipe employed in the sigma model into a Cech language. The computation in the Cech language will involve cup products of Cech cohomology groups and their maps into complex numbers. An illuminating example would be to consider a computation of the correlation function of dimension $(0,0)$ operators on the sphere. To this end, first recall from Section 5.1 that a generic dimension $(0,0)$ operator $\mathcal{O}_{i}$ with $U(1)_{\mathrm{L}} \times U(1)_{\mathrm{R}}$ charge $\left(-p_{i}, q_{i}\right)$ can be interpreted as a $\left(0, q_{i}\right)$-form with values in the bundle $\Lambda^{p_{i}} \mathcal{E}^{*}$. Thus, from Section 5.3, we find that it represents a class in the Cech cohomology group $H^{q_{i}}\left(X, \Lambda^{p_{i}} \mathcal{E}^{*}\right)$. Secondly, note that the additional $U(1)_{\mathrm{L}}$ anomaly computation of (2.7) means that for the correlation functions of our model to be non-vanishing on the sphere, they must also satisfy $\sum_{i} p_{i}=r$ in perturbation theory. Thirdly, via the fixed-point theorem 27 and the BRST transformation laws in (2.11), we find that the path integral reduces to an integral over the moduli space of holomorphic maps. Since we are considering degree-zero maps in perturbation theory, the moduli space of holomorphic maps is $X$ itself, i.e., the path integral reduces to an integral over the target space $X$. In summary, we find that a non-vanishing perturbative correlation function involving $s$ dimension $(0,0)$ operators $\mathcal{O}_{1}, \mathcal{O}_{2}, \ldots, \mathcal{O}_{s}$ on the sphere can be computed as

$$
\left\langle\mathcal{O}_{1}\left(z_{1}\right) \cdots \mathcal{O}_{s}\left(z_{s}\right)\right\rangle_{0}=\int_{X} W^{n, n}
$$

where $W^{n, n}$ is a top-degree form on $X$ which represents a class in the Cech cohomology group $H^{n}\left(X, K_{X}\right)$. This $(n, n)$-form is obtained via the 
sequence of maps

$$
\begin{aligned}
& H^{q_{1}}\left(X, \Lambda^{p_{1}} \mathcal{E}^{*}\right) \otimes \cdots \otimes H^{q_{s}}\left(X, \Lambda^{p_{s}} \mathcal{E}^{*}\right) \longrightarrow H^{n}\left(X, \otimes_{i=1}^{s} \Lambda^{p_{i}} \mathcal{E}^{*}\right) \\
& \quad \longrightarrow H^{n}\left(X, \Lambda^{r} \mathcal{E}^{*}\right) \cong H^{n}\left(X, K_{X}\right)
\end{aligned}
$$

where $\sum_{i=1}^{s} q_{i}=n$ and $\sum_{i=1}^{s} p_{i}=r$. The first map is given by the cup product of Cech cohomology classes which represent the corresponding dimension $(0,0)$ operators. The second map is given by a wedge product of holomorphic bundles. The last isomorphism follows from the required constraint $\Lambda^{r} \mathcal{E}^{*} \cong K_{X}$. Therefore, (5.17) just defines a map $H^{n}\left(X, K_{X}\right) \rightarrow \mathbb{C}$. Although this procedure is unusual for a physicist, it has been utilized in [15] as a powerful means to compute certain quantum (i.e., non-perturbative) correlation functions in the heterotic string theory. Analogous procedures follow for the computation of correlation functions involving higher dimension operators.

Note that in the computation of a non-perturbative correlation function of the above dimension $(0,0)$ operators, the operators will be represented by Cech cohomology classes in the moduli space of worldsheet instantons (see [15]). An extension of this recipe to compute the non-perturbative correlation functions of local operators of higher dimension will therefore serve as the basis of a chiral version of $(0,2)$ quantum cohomology.

\subsection{Local symmetries}

So far, we have obtained an understanding of the local structure of the $\bar{Q}_{+^{-}}$ cohomology. We shall now proceed towards our real objective of obtaining an understanding of its global structure. In order to do, we will need to glue the local descriptions that we have studied above together.

To this end, we must first cover $X$ by small open sets $\left\{U_{a}\right\}$. Recall here that in each $U_{a}$, the $\bar{Q}_{+}$-cohomology is described by the chiral algebra of local operators of a free $b c-\beta \gamma$ system on $\mathcal{E}_{f} \times U_{a}$. Next, we will need to glue these local descriptions together over the intersections $\left\{U_{a} \cap U_{b}\right\}$, so as to describe the global structure of the $\bar{Q}_{+}$-cohomology in terms of a globally defined sheaf of chiral algebras over the entire manifold $X$.

Note that the gluing has to be carried out using the automorphisms of the free $b c-\beta \gamma$ system. Thus, one must first ascertain the underlying symmetries of the system, which are in turn divided into geometrical and nongeometrical symmetries. The geometrical symmetries are used in gluing together the local sets $\left\{\mathcal{E}_{f} \times U_{a}\right\}$ into the entire holomorphic bundle $\mathcal{E} \rightarrow X$. 
The non-geometrical symmetries on the other hand, are used in gluing the local descriptions at the algebraic level.

As usual, the generators of these symmetries will be given by the charges of the conserved currents of the free $b c-\beta \gamma$ system. In turn, these generators will furnish the Lie algebra $\mathfrak{g}$ of the symmetry group. Let the elements of $\mathfrak{g}$ which generate the non-geometrical and geometrical symmetries be written as $\mathfrak{c}$ and $\mathfrak{h}=(\mathfrak{v}, \mathfrak{f})$, respectively, where $\mathfrak{v}$ generates the geometrical symmetries of $U$, while $\mathfrak{f}$ generates the fibre space symmetries of the bundle $\mathcal{E} \rightarrow U$. Since the conserved charges must also be conformally invariant, it will mean that an element of $\mathfrak{g}$ must be given by an integral of a dimension 1 current, modulo total derivatives. In addition, the currents must also be invariant under the $U(1)$ R-symmetry of action (5.14) under which the $b$ and $c$ fields have charges 1 and -1 , respectively. With these considerations in mind, the dimension 1 currents of the free $b c-\beta \gamma$ system can be constructed as follows.

Let us start by describing the currents which are associated with the geometrical symmetries first. First, if we have a holomorphic vector field $V$ on $X$ where $V=V^{i}(\gamma) \frac{\partial}{\partial \gamma^{i}}$, we can construct a $U(1)$ R-invariant dimension 1 current $J_{V}=-V^{i} \beta_{i}$. The corresponding conserved charge is then given by $K_{V}=\oint J_{V} d z$. A computation of the OPE with the elementary fields $\gamma$ gives

$$
J_{V}(z) \gamma^{k}\left(z^{\prime}\right) \sim \frac{V^{k}\left(z^{\prime}\right)}{z-z^{\prime}}
$$

Under the symmetry transformation generated by $K_{V}$, we have $\delta \gamma^{k}=i \epsilon\left[K_{V}\right.$, $\gamma^{k}$, where $\epsilon$ is an infinitesimal transformation parameter. Thus, we see from (5.19) that $K_{V}$ generates the infinitesimal diffeomorphism $\delta \gamma^{k}=i \epsilon V^{k}$ of $U$. In other words, $K_{V}$ generates the holomorphic diffeomorphisms of the target space $X$. Therefore, $K_{V}$ spans the $\mathfrak{v}$ subset of $\mathfrak{g}$. For finite diffeomorphisms, we will have a coordinate transformation $\tilde{\gamma}^{k}=g^{k}(\gamma)$, where each $g^{k}(\gamma)$ is a holomorphic function in the $\gamma^{k}$ 's. Since we are using the symmetries of the $b c-\beta \gamma$ system to glue the local descriptions over the intersections $\left\{U_{a} \cap U_{b}\right\}$, on an arbitrary intersection $U_{a} \cap U_{b}, \gamma^{k}$ and $\tilde{\gamma}^{k}$ must be defined in $U_{a}$ and $U_{b}$, respectively.

Next, let $[t(\gamma)]$ be an arbitrary $r \times r$ matrix over $X$ whose components are holomorphic functions in $\gamma$. One can then construct a $U(1) \mathrm{R}$-invariant dimension 1 current involving the fermionic fields $b$ and $c$ as $J_{F}=c^{m}[t(\gamma)]_{m}{ }^{n}$ $b_{n}$, where the indices $m$ and $n$ on the matrix $[t(\gamma)]$ denote its $(m, n)$ component, and $m, n=1,2, \ldots, r$. The corresponding conserved charge is thus given by $K_{F}=\oint J_{F} d z$. A computation of the OPE with the elementary 
fields $c$ gives

$$
J_{F}(z) c^{n}\left(z^{\prime}\right) \sim \frac{c^{m}\left(z^{\prime}\right) t_{m}^{n}}{z-z^{\prime}}
$$

while a computation of the OPE with the elementary fields $b$ gives

$$
J_{F}(z) b_{n}\left(z^{\prime}\right) \sim-\frac{t_{n}{ }^{m} b_{m}\left(z^{\prime}\right)}{z-z^{\prime}} .
$$

Under the symmetry transformation generated by $K_{F}$, we have $\delta c^{n}=i \epsilon\left[K_{F}\right.$, $\left.c^{n}\right]$ and $\delta b_{n}=i \epsilon\left[K_{F}, b_{n}\right]$. Hence, we see from (5.20) and (5.21) that $K_{F}$ generates the infinitesimal transformations $\delta c^{n}=i \epsilon c^{m} t_{m}^{n}$ and $\delta b_{n}=-i \epsilon t_{n}^{m} b_{m}$. For finite transformations, we will have $\tilde{c}^{n}=c^{m} A_{m}^{n}$ and $\tilde{b}_{n}=\left(A^{-1}\right)_{n}{ }^{m} b_{m}$, where $A$ is an $r \times r$ matrix holomorphic in $\gamma$ and given by $[A(\gamma)]=\mathrm{e}^{i \alpha[t(\gamma)]}$, where $\alpha$ is a finite transformation parameter. As before, since we are using the symmetries of the $b c-\beta \gamma$ system to glue the local descriptions over the intersections $\left\{U_{a} \cap U_{b}\right\}$, on an arbitrary intersection $U_{a} \cap U_{b},\left(c^{n}, b_{n}\right)$ and $\left(\tilde{c}^{n}, \tilde{b}_{n}\right)$ must be defined in $U_{a}$ and $U_{b}$, respectively. Recall at this point that the $c^{n}$ 's transform as holomorphic sections of the pull-back $\gamma^{*}(\mathcal{E})$, while the $b_{n}$ 's transform as holomorphic sections of the pull-back $\gamma^{*}\left(\mathcal{E}^{*}\right)$. Moreover, note that the transition function matrix of a dual bundle is simply the inverse of the transition function matrix of the original bundle. This means that we can consistently identify $[A(\gamma)]$ as the holomorphic transition matrix of the gauge bundle $\mathcal{E}$ and that $K_{\mathrm{F}}$ spans the $\mathfrak{f}$ subset of $\mathfrak{g}$. It is thus clear from the discussion so far how one can use the geometrical symmetries generated by $K_{\mathrm{V}}$ and $K_{\mathrm{F}}$ to glue the local sets $\left\{\mathcal{E}_{f} \times U_{a}\right\}$ together on intersections of small open sets to form the entire bundle $\mathcal{E} \rightarrow X$. Note, however, that $\mathfrak{h}=\mathfrak{v} \oplus \mathfrak{f}$ is not a Lie subalgebra of $\mathfrak{g}$, but only a linear subspace. This is because $\mathfrak{h}$ does not close upon itself as a Lie algebra. This leads to non-trivial consequences for $\mathfrak{g}$. In fact, this property of $\mathfrak{h}$ is related to the physical anomalies of the underlying sigma model. We will explain this as we go along. For the convenience of our later discussion, let us denote the current and charge associated with the geometrical symmetries by $J_{\mathrm{H}}=J_{\mathrm{V}}+J_{\mathrm{F}}$ and $K_{\mathrm{H}}=K_{\mathrm{V}}+K_{\mathrm{F}}$, respectively.

Before we proceed any further, note that one can also interpret the results of the last paragraph in terms of a spacetime gauge symmetry as follows. Recall that the fermionic fields $c^{n}\left(b_{n}\right)$ are identified with the matter fields $\lambda^{n}\left(\lambda_{z n}\right)$ of the underlying twisted heterotic sigma model, thus leading to their interpretation as sections of the pull-back $\gamma^{*}(\mathcal{E})\left(\gamma^{*}\left(\mathcal{E}^{*}\right)\right)$. This in turn allows us to interpret the relation $\tilde{c}^{n}=c^{m} A_{m}{ }^{n}$ as a local gauge transformation, where $[A(\gamma)]$ is the holomorphic gauge transformation matrix in the $r$-dimensional representation of the corresponding gauge group. One should then be able to find a basis of matrices such that $[t(\gamma)]=$ $\sum_{r=1}^{\operatorname{dim} \mathfrak{s}} \theta^{r}(\gamma) t_{r}$, where $\mathfrak{s}$ is the Lie algebra of the spacetime gauge group 
linearly realized by the $r$ left-moving fermi fields $\lambda^{n}$ of the sigma model, $\theta^{r}(\gamma)$ is a spacetime-dependent gauge transformation parameter, and the $t_{\mathrm{r}}$ 's are the constant generator matrices of the Lie algebra $\mathfrak{s .}[A(\gamma)]$ will then take the correct form of a gauge transformation matrix, i.e., $[A(\gamma)]=\mathrm{e}^{i \theta^{r}(\gamma) t_{r}}$.

We shall now determine the current associated with the non-geometrical symmetries. Let $B=\sum_{i} B_{i}(\gamma) d \gamma^{i}$ be a holomorphic $(1,0)$-form on $X$. We can then construct a $U(1)$ R-invariant dimension 1 current $J_{B}=B_{i} \partial_{z} \gamma^{i}$. The conserved charge is then given by $\oint J_{B} d z$. Let us assume that $B$ is an exact form on $X$, so that $B=\partial H=\partial_{i} H d \gamma^{i}$, where $H$ is some local function on $X$ that is holomorphic in $\gamma$. This in turn means that $B_{i}=$ $\partial_{i} H$. In such a case, $\oint J_{B} d z=\oint \partial_{i} H \partial_{z} \gamma^{i} d z$. From action (5.14), we have the equation of motion $\partial_{\bar{z}} \gamma^{i}=0$. Hence, $\oint J_{B} d z=\oint \partial_{i} H d \gamma^{i}=\oint d H=$ 0 by Stoke's theorem. In other words, the conserved charge constructed from $B$ vanishes if $B$ is exact and vice-versa. Let us now ascertain the conditions under which $B$ will be exact. To this end, note that it suffices to work locally on $X$, since non-local instanton effects do not contribute in perturbation theory. Via Poincare's lemma, $B$ is locally exact if and only if $B$ is a closed form on $X$, i.e., $\partial B=\partial_{i} B_{j}-\partial_{j} B_{i}=0$. Thus, for every nonvanishing holomorphic $(2,0)$-form $C=\partial B$, we will have a non-vanishing conserved charge $K_{C}=\oint J_{B} d z$. Notice that $C$ is annihilated by $\partial$ since $\partial^{2}=0$, i.e., $C$ must be a local holomorphic section of the sheaf $\Omega^{2 \text {,cl }}$. Notice also that the current $J_{B}$ is constructed from $\gamma$ and its derivatives only. Consequently, the $\gamma^{i}, b_{n}$, and $c^{n}$ fields are invariant under the symmetry transformations generated by $K_{C}$. This means that $K_{C}$ generates nongeometrical symmetries only. Hence, $K_{C}$ spans the $\mathfrak{c}$ subset of $\mathfrak{g}$.

\subsubsection{Local field transformations}

Let us now describe how the different fields of the free $b c-\beta \gamma$ system on $\mathcal{E}_{\mathrm{f}} \times U$ transform under the geometrical and non-geometrical symmetries generated by $K_{H}=K_{V}+K_{F}$ and $K_{C}$ of $\mathfrak{g}$, respectively. First, note that the symmetries generated by $K_{F}$ and $K_{C}$ act trivially on the $\gamma$ fields, i.e., the $\gamma$ fields have non-singular OPEs with $J_{F}$ and $J_{B}$. Secondly, note that the symmetries generated by $K_{V}$ and $K_{C}$ act trivially on both the $b$ and $c$ fields, i.e., the $b$ and $c$ fields have non-singular OPEs with $J_{V}$ and $J_{B}$. As for the $\beta$ fields, they transform non-trivially under all the symmetries, i.e., the OPEs of the $\beta$ fields with $J_{V}, J_{F}$, and $J_{C}$ all contain simple poles. In summary, via a computation of the relevant OPEs, we find that the fields transform under the symmetries of the free $b c-\beta \gamma$ system on $\mathcal{E}_{\mathrm{f}} \times U$ as follows:

$$
\begin{aligned}
& \tilde{\gamma}^{i}=g^{i}(\gamma), \\
& \tilde{\beta}_{i}=\beta_{k} D^{k}{ }_{i}+b_{m} c^{n} A_{n}{ }^{l} D^{k}{ }_{i}\left(\partial_{k} A^{-1}\right)_{\mathrm{L}}{ }^{m}+\partial_{z} \gamma^{j} E_{i j},
\end{aligned}
$$




$$
\begin{aligned}
& \tilde{c}^{n}=c^{m} A_{m}{ }^{n}, \\
& \tilde{b}_{n}=\left(A^{-1}\right)_{n}{ }^{m} b_{m},
\end{aligned}
$$

where $i, j, k=1,2, \ldots, N=\operatorname{dim}_{\mathbb{C}} X$, and $l, m, n=1,2, \ldots, r$. Here, $D$ and $E$ are $N \times N$ matrices such that $[D]^{\mathrm{T}}=[\partial g]^{-1}$ and $[E]=[\partial B]$, that is, $\left[\left(D^{\mathrm{T}}\right)^{-1}\right]_{i}{ }^{k}=\partial_{i} g^{k}$ and $[E]_{i j}=\partial_{i} B_{j}$. It can be verified that $\tilde{\beta}, \tilde{\gamma}, \tilde{b}$, and $\tilde{c}$ obey the correct OPEs among themselves. We thus conclude that the fields must undergo the above transformations (5.22)-(5.25) when we glue a local description (in a small open set) to another local description (in another small open set) on the mutual intersection of open sets using the automorphism of the free $b c-\beta \gamma$ system. Note that the last term in $\tilde{\beta}$ is due to the non-geometrical symmetry transformation generated by $K_{C}$, while the first and second term in $\tilde{\beta}$ is due to the geometrical symmetry transformation generated by $K_{V}$ and $K_{F}$, respectively. This observation will be important when we discuss what happens at the $(2,2)$ locus later.

Another important comment to be made is that in computing (5.22)(5.25), we have just rederived, from a purely physical perspective, the set of field transformations $(7.2 \mathrm{a})-(7.2 \mathrm{~d})$ in [3], which defines the valid automorphisms of the sheaf of vertex superalgebras obtained from a mathematical model that is equivalent to a free $b c-\beta \gamma$ system with action (5.14). Hence, we learn that the sheaf $\widehat{\mathcal{A}}$ is mathematically known as a sheaf of vertex superalgebras spanned by CDOs on the exterior algebra $\Lambda \mathcal{E}=\oplus_{i=1}^{r k(\mathcal{E})} \Lambda^{i} \mathcal{E}$ of the holomorphic vector bundle $\mathcal{E}$ over $X[3,5]$.

\subsubsection{A non-trivial extension of Lie algebras and groups}

We shall now study the properties of the symmetry algebra $\mathfrak{g}$ of the free $b c-\beta \gamma$ system on $U$. From the analysis thus far, we find that we can write $\mathfrak{g}=\mathfrak{c}+\mathfrak{h}$ as a linear space, where $\mathfrak{h}=\mathfrak{v}+\mathfrak{f}$. Note that $\mathfrak{c}$ is a trivial abelian subalgebra of $\mathfrak{g}$. This because the commutator of $K_{C}$ with itself vanishes the OPE of $J_{B}$ with itself is non-singular since the current is constructed from $\gamma$ and its derivatives only. Hence, $\mathfrak{g}$ can be expressed in an extension of Lie algebras as follows:

$$
0 \longrightarrow \mathfrak{c} \longrightarrow \mathfrak{g} \longrightarrow \mathfrak{h} \longrightarrow 0
$$

In fact, (5.26) is an exact sequence of Lie algebras, as we will show shortly that $[\mathfrak{h}, \mathfrak{c}] \subset \mathfrak{c}$. This means that $\mathfrak{c}$ is "forgotten" when we project $\mathfrak{g}$ onto $\mathfrak{h}$. 
The action of $\mathfrak{h}$ on $\mathfrak{c}$ can be found from the $J_{H}(z) J_{C}\left(z^{\prime}\right)$ OPE

$$
\begin{aligned}
& {\left[-V^{i} \beta_{i}(z)+c^{m} t_{m}^{n} b_{n}(z)\right] \cdot B_{j} \partial_{z^{\prime}} \gamma^{j}\left(z^{\prime}\right) \sim \frac{1}{z-z^{\prime}}\left[V^{i}\left(\partial_{i} B_{k}-\partial_{k} B_{i}\right)\right.} \\
& \left.\quad+\partial_{k}\left(V^{i} B_{i}\right)\right] \partial_{z^{\prime}} \gamma^{k}+\frac{1}{\left(z-z^{\prime}\right)^{2}} V^{i} B_{i}\left(z^{\prime}\right) .
\end{aligned}
$$

The commutator of $K_{H}$ with $K_{C}$, and thus $[\mathfrak{h}, \mathfrak{c}]$, is simply the residue of the simple pole on the RHS of (5.27). The numerator of the first term on the RHS of (5.27), given by $V^{i}\left(\partial_{i} B_{k}-\partial_{k} B_{i}\right)+\partial_{k}\left(V^{i} B_{i}\right)$, is the same as $\left(\mathcal{L}_{V}(B)\right)_{k}$, the $k$ th component of the one-form that results from the action of a Lie derivative of the vector field $V$ on the one-form $B$. This observation should not come as a surprise since the charges of $J_{V}$ generate diffeomorphisms of $U$, and only the $J_{V} \cdot J_{C}$ part of the OPE in (5.27) is non-trivial (since $J_{F}$ has non-singular OPEs with $J_{C}$ ). Hence, $[\mathfrak{h}, \mathfrak{c}] \subset \mathfrak{c}$ as claimed.

Let us now compute the commutator of two elements of $\mathfrak{h}$. To this end, let $V$ and $W$ be two vector fields on $U$ that are holomorphic in $\gamma$. Let $t(\gamma)$ and $\tilde{t}(\gamma)$ be $r \times r$ matrices holomorphic in $\gamma$. Let $V$ and $W$ be associated with the currents $J_{V}(z) \subset J_{H}(z)$ and $J_{W}\left(z^{\prime}\right) \subset J_{H}\left(z^{\prime}\right)$, respectively. Likewise, let $t$ and $\tilde{t}$ be associated with the currents $J_{F}(z) \subset J_{H}(z)$ and $J_{\widetilde{F}}\left(z^{\prime}\right) \subset J_{H}\left(z^{\prime}\right)$, respectively. The $J_{H}(z) J_{H}\left(z^{\prime}\right)$ OPE is then computed to be

$$
\begin{array}{r}
J_{H}(z) J_{H}\left(z^{\prime}\right) \sim-\frac{\left(V^{i} \partial_{i} W^{j}-W^{i} \partial_{i} V^{j}\right) \beta_{j}}{z-z^{\prime}}-\frac{\left(\partial_{k} \partial_{j} V^{i}\right)\left(\partial_{i} W^{j} \partial_{z^{\prime}} \gamma^{k}\right)}{z-z^{\prime}} \\
+\frac{c^{m}\{t, \tilde{t}\}_{m}^{c} b_{c}}{z-z^{\prime}}+\frac{\operatorname{Tr}\left[\tilde{t} \partial_{i} t\right] \partial_{z^{\prime}} \gamma^{i}}{z-z^{\prime}}-\frac{\partial_{j} V^{i} \partial_{i} W^{j}\left(z^{\prime}\right)}{\left(z-z^{\prime}\right)^{2}}+\frac{\operatorname{Tr}[\tilde{t} t]\left(z^{\prime}\right)}{\left(z-z^{\prime}\right)^{2}} .
\end{array}
$$

The last two terms on the RHS of (5.28), being double poles, do not contribute to the commutator. From the mathematical relation $[V, W]^{j}=$ $\left(\mathcal{L}_{V}(W)\right)^{j}=V^{i} \partial_{i} W^{k}-W^{i} \partial_{i} V^{j}$, we see that the first term takes values in $\mathfrak{v} \subset \mathfrak{h}$, the second term takes values in $\mathfrak{c}$, the third term takes values in $\mathfrak{f} \subset \mathfrak{h}$, and the fourth term takes values in $\mathfrak{c}$. The first and third terms, which come from a single contraction of elementary fields in evaluating the $\mathrm{OPE}$, arise from the expected results $J_{V}(z) J_{W}\left(z^{\prime}\right) \sim J_{[V, W]} /\left(z-z^{\prime}\right)$ and $J_{F}(z) J_{\widetilde{F}}\left(z^{\prime}\right) \sim J_{\{t, \tilde{t}\}} /\left(z-z^{\prime}\right)$, respectively. We would have obtained the same results by computing the commutator of $J_{V}$ and $J_{W}$, and that of $J_{F}$ and $J_{\widetilde{F}}$, via Poisson brackets in the classical $b c-\beta \gamma$ theory. The second and fourth terms are the reason why $[\mathfrak{h}, \mathfrak{h}] \nsubseteq \mathfrak{h}$. Note that these two terms result from multiple contractions of elementary fields, just like the anomalies of conformal field theory. Hence, since $\mathfrak{h}$ does not close upon itself as a Lie algebra, $\mathfrak{g}$ is not a semi-direct product of $\mathfrak{h}$ and $\mathfrak{c}$. Consequently, the extension of Lie algebras in (5.26) is non-trivial. Is the non-triviality of the extension of Lie algebras of the symmetries of the $b c-\beta \gamma$ system on $\mathcal{E}_{f} \times U$ 
then related to the physical anomalies of the underlying sigma model? Let us study this further.

The exact sequence of Lie algebras in (5.26) will result in the following group extension when we exponentiate the elements of $\mathfrak{g}$ :

$$
1 \longrightarrow \widetilde{C} \longrightarrow \widetilde{G} \longrightarrow \widetilde{H} \longrightarrow 1 \text {. }
$$

Here, $\widetilde{G}$ is the symmetry group of all admissible automorphisms of the $b c-\beta \gamma$ system, $\widetilde{C}$ is the symmetry group of the non-geometrical automorphisms, and $\widetilde{H}$ is the symmetry group of the geometrical automorphisms. Just as in (5.26), (5.29) is an exact sequence of groups, i.e., the kernel of the map $\widetilde{G} \rightarrow \widetilde{H}$ is given by $\widetilde{C}$. This means that the non-geometrical symmetries are "forgotten" when we project the full symmetries onto the geometrical symmetries. Since (5.29) is derived from a non-trivial extension of Lie algebras in (5.26), it will be a non-trivial group extension. In fact, the cohomology class of the group extension that captures its non-triviality is given by [3]

$$
c_{1}^{2}-2 c_{2}-\left(c_{1}^{\prime 2}-2 c_{2}^{\prime}\right) \in H^{2}\left(\widetilde{H}, \Omega_{\widetilde{H}}^{2, c l}\right),
$$

where $\Omega_{\widetilde{H}}^{2, \text { cl }}$ is a sheaf of an $\widetilde{H}$-module of closed two-forms, and $c_{i}, c_{i}^{\prime} \in$ $H^{i}\left(\widetilde{H}, \Omega_{\widetilde{H}}^{2, \text { cl }}\right)$ are the universal Chern classes. The cohomology class $H^{2}(\widetilde{H}$, $\left.\Omega_{\widetilde{H}}^{2, \text { cl }}\right)$ vanishes if and only if the kernel of the map $\widetilde{G} \rightarrow \widetilde{H}$ is empty, i.e., $\widetilde{G}=\widetilde{H}$. Thus, the group extension is trivial if the admissible automorphisms of the $b c-\beta \gamma$ system are solely of a geometrical kind. This observation will be essential to our discussion of the sigma model at the $(2,2)$ locus later. Let us return back to the issue of (5.30)'s relevance to the physical anomalies of the underlying sigma model. Note that the mathematical arguments in [3] and a detailed computation in [5] show that (5.29), together with its cohomology class (5.30), implies that the obstruction to a globally defined sheaf of chiral algebras $\widehat{\mathcal{A}}$ of CDOs on the exterior algebra $\Lambda \mathcal{E}$ must be captured by the cohomology class

$$
2 \operatorname{ch}_{2}(T X)-2 \operatorname{ch}_{2}(\mathcal{E})
$$

which in turn represents an element of $H^{2}\left(X, \Omega_{X}^{2, c l}\right)$ (as explained in footnote 13 of Section 4). Notice that the vanishing of (5.31) coincides with one of the anomaly-cancellation conditions of the underlying twisted heterotic sigma model in (4.1). In hindsight, this "coincidence" should not be entirely surprising - note that a physically valid sigma model must be defined over all of $\mathcal{E} \rightarrow X$ (and $\Sigma$ ). Since (5.31) captures the obstruction to gluing the local descriptions together to form a global description, this implies that the sigma model, which is described locally by the free $b c-\beta \gamma$ system on $\mathcal{E}_{\mathrm{f}} \times U$, cannot be globally defined over all of $\mathcal{E} \rightarrow X$ unless (5.31) vanishes. Hence, the anomaly which obstructs the physical validity of the underlying sigma 
model must be given by (5.31). Thus, the non-triviality of the extension of Lie algebras of the symmetries of the $b c-\beta \gamma$ system on $\mathcal{E}_{\mathrm{f}} \times U$ is indeed related to the physical anomaly of the underlying sigma model.

\subsection{Gluing the local descriptions together}

Now, we will describe explicitly, how one can glue the local descriptions together using the automorphisms of the free $b c-\beta \gamma$ system on $\mathcal{E}_{\mathrm{f}} \times U$ to obtain a globally defined sheaf of chiral algebras. In the process, we will see how the cohomology class in (5.31) emerges as an obstruction to gluing the locally defined sheaves of chiral algebras globally on $X$. Moreover, we can also obtain the other anomaly in (4.1), which is not captured in (5.31) (for reasons we will explain shortly), when we consider gluing the sheaves of chiral algebras globally over $X$ and $\Sigma$. In addition, we will see that the moduli of the resulting sheaf emerge as a Cech cohomology class generated by a relevant Cech cocycle.

To begin with, let us take a suitable collection of small open sets $U_{a} \subset \mathbb{C}^{n}$, where $n=\operatorname{dim}_{\mathbb{C}} X$. Next, consider the corresponding set of product spaces $\left\{\mathcal{E}_{\mathrm{f}} \times U_{a}\right\}$. We want to glue these trivial product spaces together to make a good cover of the holomorphic vector bundle $\mathcal{E} \rightarrow X$. On each $U_{a}$, the sheaf $\widehat{\mathcal{A}}$ of chiral algebras is defined by a free $b c-\beta \gamma$ system on $\left\{\mathcal{E}_{\mathrm{f}} \times U_{a}\right\}$. We need to glue together these free conformal field theories to get a globally defined sheaf of chiral algebras.

It will be convenient for us to first describe how we can geometrically glue the set of trivial product spaces $\left\{\mathcal{E}_{\mathrm{f}} \times U_{a}\right\}$ together to form the bundle $\mathcal{E} \rightarrow X$. For each $a, b$, let us pick a product space $\mathcal{E}_{\mathrm{f}} \times U_{a b} \subset \mathcal{E}_{\mathrm{f}} \times U_{a}$ and likewise another product space $\mathcal{E}_{\mathrm{f}} \times U_{b a} \subset \mathcal{E}_{\mathrm{f}} \times U_{b}$. Let us define a geometrical symmetry $\hat{h}_{a b}$ (given by a product of holomorphic diffeomorphisms on $U$ with holomorphic homeomorphisms of the fibre $\mathcal{E}_{\mathrm{f}}$ ) between these product spaces as

$$
\hat{h}_{a b}: \mathcal{E}_{\mathrm{f}} \times U_{a b} \cong \mathcal{E}_{f} \times U_{b a} .
$$

Note that $\hat{h}$ can be viewed as a geometrical gluing operator corresponding to an element of the geometrical symmetry group $\widetilde{H}$. From the above definition, we see that $\hat{h}_{b a}=\hat{h}_{a b}^{-1}$. We want to identify an arbitrary point $P \in \mathcal{E}_{\mathrm{f}} \times U_{a b}$ with an arbitrary point $Q \in \mathcal{E}_{\mathrm{f}} \times U_{b a}$ if $Q=\hat{h}_{a b}(P)$. This identification will be consistent if for any $U_{a}, U_{b}$, and $U_{c}$, we have

$$
\hat{h}_{c a} \hat{h}_{b c} \hat{h}_{a b}=1
$$


in any triple intersection $U_{a b c}$ over which all the maps $\hat{h}_{c a}, \hat{h}_{b c}$, and $\hat{h}_{a b}$ are defined. The relation in (5.33) tells us that the different pieces $\mathcal{E}_{\mathrm{f}} \times U_{a}$ can be glued together via the set of maps $\left\{\hat{h}_{a b}\right\}$ to make a holomorphic vector bundle $\mathcal{E} \rightarrow X$. The holomorphic and complex structure moduli of the bundle and its base will then manifest as parameters in the $\hat{h}_{a b}$ 's.

Suppose we now have a sheaf of chiral algebras on each $U_{a}$, and we want to glue them together on overlaps to get a sheaf of chiral algebras on $X$. The gluing must be done using the automorphisms of the conformal field theories. Thus, for each pair $U_{a}$ and $U_{b}$, we select a conformal field theory symmetry $\hat{g}_{a b}$ that maps the free $b c-\beta \gamma$ system on $\mathcal{E}_{\mathrm{f}} \times U_{a}$, restricted to $\mathcal{E}_{\mathrm{f}} \times U_{a b}$, to the free $b c-\beta \gamma$ system on $\mathcal{E}_{\mathrm{f}} \times U_{b}$, similarly restricted to $\mathcal{E}_{\mathrm{f}} \times U_{b a}$. We get a globally defined sheaf of chiral algebras if the gluing is consistent:

$$
\hat{g}_{c a} \hat{g}_{b c} \hat{g}_{a b}=1 \text {. }
$$

Note that $\hat{g}$ can be viewed as a gluing operator corresponding to an element of the full symmetry group $\widetilde{G}$. As usual, we have $\hat{g}_{b a}=\hat{g}_{a b}^{-1}$. Moreover, recall at this point that from the exact sequence of groups in (5.29), we have a map $\widetilde{G} \rightarrow \widetilde{H}$ which "forgets" the non-geometrical symmetry group $\widetilde{C} \subset \widetilde{G}$. As such, for any arbitrary set of $\hat{g}$ 's which obey (5.34), the geometrical condition (5.33) will be automatically satisfied, regardless of what the non-geometrical gluing operator $\hat{c}$ corresponding to an element of $\widetilde{C}$ is. Hence, every possible way to glue the conformal field theories together via $\hat{g}$ determines a way to geometrically glue the set of product spaces $\left\{\mathcal{E}_{\mathrm{f}} \times U_{a}\right\}$ together to form a unique holomorphic vector bundle $\mathcal{E} \rightarrow X$ over which one defines the resulting conformal field theory.

The above discussion translates to the fact that for a given set of $\hat{h}_{a b}$ 's which obey (5.33), the corresponding set of $\hat{g}_{a b}$ 's which obey (5.34) are not uniquely determined; for each $U_{a b}$, we can still pick an element $\mathcal{C}_{a b} \in$ $H^{0}\left(U_{a b}, \Omega^{2, c l}\right)$, which represents an element of $\mathfrak{c}$ (as discussed in Section 5.5), so that $\exp \left(\mathcal{C}_{a b}\right)$ represents an element of $\widetilde{C}$. One can then transform $\hat{g}_{a b} \rightarrow$ $\hat{g}_{a b}^{\prime}=\exp \left(\mathcal{C}_{a b}\right) \hat{g}_{a b}$, where $\hat{g}_{a b}^{\prime}$ is another physically valid gluing operator. The condition that the gluing identity (5.34) is obeyed by $\hat{g}^{\prime}$, i.e., $\hat{g}_{c a}^{\prime} \hat{g}_{b c}^{\prime} \hat{g}_{a b}^{\prime}=1$, is that in each triple intersection $U_{a b c}$, we should have

$$
\mathcal{C}_{c a}+\mathcal{C}_{b c}+\mathcal{C}_{a b}=0 .
$$

From $\hat{g}_{b a}^{\prime}=\left(\hat{g}_{a b}^{\prime}\right)^{-1}$, we have $\mathcal{C}_{a b}=-\mathcal{C}_{b a}$. Moreover, $\widetilde{\mathcal{C}}_{a b} \sim \mathcal{C}_{a b}+\mathcal{S}_{a}-\mathcal{S}_{b}$ for some $\mathcal{S}$, in the sense that the $\widetilde{\mathcal{C}}$ 's will obey (5.35) as well. In other words, the $\mathcal{C}$ 's in (5.35) must define an element of the Cech cohomology group $H^{1}\left(X, \Omega_{X}^{2, \mathrm{cl}}\right)$. As usual, $\exp \left(\mathcal{C}_{a b}\right)$ is "forgotten" when we project from $\hat{g}_{a b}^{\prime}$ to the geometrical gluing operator $\hat{h}_{a b}$. Therefore, in going from $\hat{g}$ to $\hat{g}^{\prime}$, the symmetry $\hat{h}$, and consequently the bundle $\mathcal{E} \rightarrow X$, remains unchanged. 
Now, let us use a specific $\hat{g}$ operator to define the specific symmetries of a free $b c-\beta \gamma$ system, which in turn will define a unique sheaf of chiral algebras. In this sense, given any sheaf and an element $\mathcal{C} \in H^{1}\left(X, \Omega_{X}^{2, \mathrm{cl}}\right)$, one can define a new sheaf by going from $\hat{g} \rightarrow \exp (\mathcal{C}) \hat{g}$. So, via the action of $H^{1}\left(X, \Omega_{X}^{2, \text { cl }}\right)$, we get a family of sheaves of chiral algebras, with the same target space $\mathcal{E} \rightarrow X$. Hence, the moduli of the sheaf of chiral algebras are represented by a class in $H^{1}\left(X, \Omega_{X}^{2, c l}\right)$. Together with the results of Section 3.3, we learn that the analysis of a family of sheaves of chiral algebras on a unique Kähler target space $X$ is equivalent to the analysis of a unique sheaf of chiral algebras on a family $\left\{X^{\prime}\right\}$ of non-Kähler target spaces.

\subsubsection{The anomaly}

We now move on to discuss the case when there is an obstruction to the gluing. Essentially, the obstruction occurs when (5.34) is not satisfied by the $\hat{g}$ 's. In such a case, one generally has, on triple intersections $U_{a b c}$, the following relation

$$
\hat{g}_{c a} \hat{g}_{b c} \hat{g}_{a b}=\exp \left(\mathcal{C}_{a b c}\right)
$$

for some $\mathcal{C}_{a b c} \in H^{0}\left(U_{a b c}, \Omega^{2, \mathrm{cl}}\right)$. The reason for (5.36) is as follows. First, note that the LHS of (5.36) projects purely to the group of geometrical symmetries associated with $\hat{h}$. If the bundle $\mathcal{E} \rightarrow X$ is to exist mathematically, there will be no obstruction to its construction, i.e., the LHS of (5.36) will map to the identity under the projection. Hence, the RHS of (5.36) must represent an element of the abelian group $\widetilde{C}$ (generated by $\mathfrak{c}$ ) that acts trivially on the coordinates $\gamma^{i}$ of $U_{a}$ 's and the local sections $c^{m}$ of $\left(\mathcal{E}_{\mathrm{f}} \times U_{a}\right)$ 's.

Recall that the choice of $\hat{g}_{a b}$ was not unique. If we transform $\hat{g}_{a b} \rightarrow$ $\exp \left(\mathcal{C}_{a b}\right) \hat{g}_{a b}$ via a (non-geometrical) symmetry of the system, we get

$$
\mathcal{C}_{a b c} \rightarrow \mathcal{C}_{a b c}^{\prime}=\mathcal{C}_{a b c}+\mathcal{C}_{c a}+\mathcal{C}_{b c}+\mathcal{C}_{a b}
$$

If it is possible to pick $\mathcal{C}_{a b}$ 's to set all $\mathcal{C}_{a b c}^{\prime}=0$, then there is no obstruction to gluing and one can obtain a globally defined sheaf of chiral algebras.

In any case, in quadruple overlaps $U_{a} \cap U_{b} \cap U_{c} \cap U_{d}$, the $\mathcal{C}$ 's obey

$$
\mathcal{C}_{a b c}-\mathcal{C}_{b c d}+\mathcal{C}_{c d a}-\mathcal{C}_{d a b}=0 .
$$

Together with the equivalence relation (5.37), this means that the $\mathcal{C}$ 's in (5.38) must define an element of the Cech cohomology group $H^{2}\left(X, \Omega_{X}^{2, \text { cl }}\right)$. In other words, the obstruction to gluing the locally defined sheaves of chiral algebras is captured by a non-vanishing cohomology class $H^{2}\left(X, \Omega_{X}^{2, c l}\right)$. As discussed in Section 4 and the last paragraph of Section 5.5, this class can be represented in de Rham cohomology by $2\left[\operatorname{ch}_{2}(T X)-\operatorname{ch}_{2}(\mathcal{E})\right]$. Thus, we 
have obtained an interpretation of the anomaly in the twisted heterotic sigma model in terms of an obstruction to a global definition of the sheaf of chiral algebras derived from a free $b c-\beta \gamma$ system that describes the sigma model locally on $X$.

\subsubsection{The other anomaly}

In Section 4, we showed that the twisted heterotic sigma model had two anomalies, one involving $\operatorname{ch}_{2}(\mathcal{E})-\operatorname{ch}_{2}(T X)$ and the other involving $\frac{1}{2} c_{1}(\Sigma)$ $\left(c_{1}(\mathcal{E})-c_{1}(T X)\right)$. We have already seen how the first anomaly arises from the Cech perspective. How then can we see the second anomaly in the present context?

So far, we have constructed a sheaf of chiral algebras globally on $X$ but only locally on the worldsheet $\Sigma$. This is because the chiral algebra of the twisted heterotic sigma model is not invariant under holomorphic reparameterizations of the worldsheet coordinates at the quantum level ${ }^{21}$ and, as such, can only be given a consistent definition locally on an arbitrary Riemann surface $\Sigma$. Since $c_{1}(\Sigma)$ can be taken to be zero when we work locally on $\Sigma$, the second anomaly vanishes and therefore, we will not get to see it.

Now note that the free $b c-\beta \gamma$ system is conformally invariant; in other words, it can be defined globally on an arbitrary Riemann surface $\Sigma$. But notice that the anomaly that we are looking for is given by $\frac{1}{2} c_{1}(\Sigma)\left(c_{1}(\mathcal{E})-\right.$ $\left.c_{1}(T X)\right)$. Hence, it will vanish even if we use a free $b c-\beta \gamma$ system that can be globally defined on $\Sigma$ if we continue to work locally on the bundle $\mathcal{E} \rightarrow X$, where $c_{1}(\mathcal{E})=c_{1}(T X)=0$. Therefore, the only way to see the second anomaly is to work globally on both $X$ (and hence $\mathcal{E} \rightarrow X$ ) and $\Sigma$. (In fact, recall that the underlying sigma model is physically defined on all of $\Sigma$ and $\mathcal{E} \rightarrow X$.) We shall describe how to do this next.

Let us cover $\Sigma$ and $X$ with small open sets $\left\{P_{\tau}\right\}$ and $\left\{U_{a}\right\}$, respectively. This will allow us to cover $\mathcal{E} \times \Sigma$ with open sets $W_{a \tau}=\mathcal{E}_{\mathrm{f}} \times U_{a} \times P_{\tau}$. On each $P_{\tau}$, we can define a free $b c-\beta \gamma$ system with target $\mathcal{E}_{\mathrm{f}} \times U_{a}$. In other words, on each open set $W_{a \tau}$, we define a free $b c-\beta \gamma$ system and hence a sheaf of chiral algebras. What we want to do is to glue the sheaves of chiral algebras on the $\left(\mathcal{E}_{\mathrm{f}} \times U_{a} \times P_{\tau}\right)$ 's together on overlaps to get a globally defined sheaf of chiral algebras, with target space $\mathcal{E} \rightarrow X$, defined on all of $\Sigma$. As before, the gluing must be done using the admissible automorphisms of the free $b c-\beta \gamma$ system.

\footnotetext{
${ }^{21}$ To see this, recall from Section 3.1 that in the quantum theory, the holomorphic stress tensor $T_{z z}$ is not in the $\bar{Q}_{+}$-cohomology (i.e., $\left\{\bar{Q}_{+}, T_{z z}\right\} \neq 0$ ) unless we have a stable bundle $\mathcal{E}$ with $c_{1}(X)=0$. This prevents the $\bar{Q}_{+}$-cohomology and thus the chiral algebra from being invariant under arbitrary reparameterizations of $\Sigma$.
} 
Recall from Section 5.5 that the admissible automorphisms are given by the symmetry group $\widetilde{G}$. Note that the set of geometrical symmetries $\widetilde{H} \subset \widetilde{G}$ considered in Section 5.5 can be extended to include holomorphic diffeomorphisms of the worldsheet $\Sigma$ - as mentioned above, the free $b c-\beta \gamma$ system is conformally invariant and is therefore invariant under arbitrary holomorphic reparameterizations of the coordinates on $\Sigma$. Previously in Section 5.5, there was no requirement to consider and exploit this additional geometrical symmetry in gluing the local descriptions together simply because we were working locally on $\Sigma$. Then, gluing of the local descriptions at the geometrical level was carried out using $\widetilde{H}$, where $\widetilde{H}$ consists of the group of holomorphic diffeomorphisms of $X$ and the group of holomorphic homeomorphisms of the fibre $\mathcal{E}_{\mathrm{f}}$. Now that we want to work globally on $\Sigma$ as well, one will need to use the symmetry of the free conformal field theory under holomorphic diffeomorphisms of $\Sigma$ to glue the $P_{\tau}$ 's together to form $\Sigma$. In other words, gluing of the local descriptions at the geometrical level must now be carried out using the geometrical symmetry group $\widetilde{H}^{\prime}$, where $\widetilde{H}^{\prime}$ consists of the group of holomorphic diffeomorphisms on $\Sigma$ and $X$ and the group of holomorphic homeomorphisms of the fibre $\mathcal{E}_{\mathrm{f}}$. Now, let the conformal field theory gluing map from $W_{a \tau}$ to $W_{b \nu}$ be given by $\hat{g}_{a \tau, b \nu}$. Let the corresponding geometrical and non-geometrical gluing maps from $W_{a \tau}$ to $W_{b \nu}$ be given by $\hat{h}_{a \tau, b \nu}^{\prime}$ and $\hat{c}_{a \tau, b \nu}^{\prime}$, respectively. Since we have a sensible notion of a holomorphic map $\gamma: \Sigma \rightarrow X$, and the bundle $\mathcal{E}$ and worldsheet $\Sigma$ are defined to exist mathematically, there is no obstruction to gluing at the geometrical level, i.e.,

$$
\hat{h}_{c \sigma, a \tau}^{\prime} \hat{h}_{b \nu, c \sigma}^{\prime} \hat{h}_{a \tau, b \nu}^{\prime}=1
$$

in triple intersections. There will be no obstruction to gluing at all levels if one has the relation

$$
\hat{g}_{c \sigma, a \tau} \hat{g}_{b \nu, c \sigma} \hat{g}_{a \tau, b \nu}=1 .
$$

However, (5.40) may not always be satisfied. Similar to our previous arguments concerning the anomaly $2 \operatorname{ch}_{2}(T X)-2 \operatorname{ch}_{2}(\mathcal{E}) \in H^{2}\left(X, \Omega_{X}^{2, \text { cl }}\right)$, since one has a map $\hat{g}_{a \tau, b \nu} \rightarrow \hat{h}_{a \tau, b \nu}^{\prime}$ in which $\hat{c}_{a \tau, b \nu}^{\prime}$ is "forgotten", in general, we will have

$$
\hat{g}_{c \sigma, a \tau} \hat{g}_{b \nu, c \sigma} \hat{g}_{a \tau, b \nu}=\exp \left(\mathcal{C}_{a \tau b \nu c \sigma}\right),
$$

where the $\mathcal{C}_{a \tau b \nu c \sigma}$ 's on any triple overlap define a class in the two-dimensional Cech cohomology group $H^{2}(X \times \Sigma, \mathcal{G})$. $\mathcal{G}$ is a sheaf associated with the nongeometrical symmetries of the free $b c-\beta \gamma$ system. Being non-geometrical in nature, these symmetries will act trivially on the $\gamma^{i}$ coordinates of $X$ and the sections $c^{m}$ (and $b_{m}$ ) of the pull-back $\gamma^{*}(\mathcal{E})\left(\right.$ and $\left.\gamma^{*}\left(\mathcal{E}^{*}\right)\right)$.

Earlier on in our discussion, when we worked locally on $\Sigma$ but globally on $X$, we constructed a $U(1)$ R-invariant dimension 1 current $J_{B}$ from a 
(1,0)-form $B$ on $X$, whose conserved charge $K_{C}$ was shown to generate the non-geometrical symmetries of the free $b c-\beta \gamma$ conformal field theory. Therefore, if one works globally on both $\Sigma$ and $X$, one will need to construct an analogous $U(1)$ R-invariant dimension 1 current $J_{B^{\prime}}$ from a $(1,0)$-form $B^{\prime}$ on $X \times \Sigma$, such that the corresponding conformally invariant conserved charge will generate the non-geometrical symmetries in this extended case. Since the current $J_{B^{\prime}}$ should have non-singular OPEs with the $\gamma, c$, and $b$ fields, it can only depend linearly on $\partial_{z} \gamma$ and be holomorphic in $\gamma$ and $z$. Thus, the non-geometrical symmetries will be generated by the conserved charge $\oint J_{B^{\prime}} d z$, with

$$
J_{B^{\prime}}=B_{i}(\gamma, z) \partial_{z} \gamma^{i}+B_{\Sigma}(\gamma, z) .
$$

Here, $B_{i}$ and $B_{\Sigma}$ are components of a holomorphic (1,0)-form $B^{\prime}=B_{i} d \gamma^{i}+$ $B_{\Sigma} d z$ on $X \times \Sigma$, where $B_{i}$ and $B_{\Sigma}$ have scaling dimensions 0 and 1 , respectively, i.e., for $z \rightarrow \tilde{z}=\lambda z$, we have $B_{i}(\gamma, z) \rightarrow B_{i}(\gamma, \tilde{z})=B_{i}(\gamma, z)$ and $B_{\Sigma}(\gamma, z) \rightarrow B_{\Sigma}(\gamma, \tilde{z})=\lambda^{-1} B_{\Sigma}(\gamma, z)$.

If $B^{\prime}$ is exact, i.e., $B^{\prime}=\partial H^{\prime}$ for some local function $H^{\prime}(\gamma, z)$ on $X \times \Sigma$ holomorphic in $\gamma$ and $z$, we will have $B_{i}=\partial_{i} H^{\prime}$ and $B_{\Sigma}=\partial_{z} H^{\prime}$. As a result, the conserved charge $\oint J_{B^{\prime}} d z=\oint\left(\partial_{i} H^{\prime}\right) d \gamma^{i}+\left(\partial_{z} H^{\prime}\right) d z=\oint d H^{\prime}=0$ by Stoke's theorem. Using the same arguments found in Section 5.5 (where we discussed the conserved charge $K_{C}$ ), we learn that for every non-vanishing holomorphic $(2,0)$-form $C^{\prime}=\partial B^{\prime}$ on $X \times \Sigma$, we will have a non-vanishing conserved charge $K_{C^{\prime}}=\oint J_{B^{\prime}} d z$. Since $C^{\prime}$ is $\partial$-closed, it is a local holomorphic Section of $\Omega_{X \times \Sigma}^{2, \mathrm{cl}}$. Therefore, we find that the sheaf associated with the non-geometrical symmetries that act trivially on $\gamma, c$, and $b$ is isomorphic to $\Omega_{X \times \Sigma}^{2, c l}$. Thus, the obstruction to a globally defined sheaf of chiral algebras, with target space $\mathcal{E} \rightarrow X$, defined on all of $\Sigma$, will be captured by a class in the Cech cohomology group $H^{2}\left(X \times \Sigma, \Omega_{X \times \Sigma}^{2, \mathrm{cl}}\right)$. Hence, the physical anomalies of the underlying sigma model ought to be captured by the de Rham cohomology classes which take values in $H^{2}\left(X \times \Sigma, \Omega_{X \times \Sigma}^{2, \mathrm{cl}}\right)$.

In fact, since $\Sigma$ is of complex dimension 1 , its space of $(2,0)$-forms vanishes. Thus, we will have $\Omega_{X \times \Sigma}^{2, \mathrm{cl}}=\left(\Omega_{X}^{2, \mathrm{cl}} \otimes \mathcal{O}_{\Sigma}\right) \oplus\left(\Omega_{X}^{1, \mathrm{cl}} \otimes \Omega_{\Sigma}^{1, \mathrm{cl}}\right)$ (where $\mathcal{O}_{\Sigma}$ is the sheaf of holomorphic functions on $\Sigma$ ). In other words, on a compact Riemann surface $\Sigma$, where the only holomorphic functions over it are constants, i.e., $H^{0}(\Sigma, \mathcal{O}) \cong \mathbb{C}$, we have the expansion

$$
H^{2}\left(X \times \Sigma, \Omega_{X \times \Sigma}^{2, \mathrm{cl}}\right)=H^{2}\left(X, \Omega_{X}^{2, \mathrm{cl}}\right) \oplus\left(H^{1}\left(X, \Omega_{X}^{1, \mathrm{cl}}\right) \otimes H^{1}\left(\Sigma, \Omega_{\Sigma}^{1, \mathrm{cl}}\right)\right) \oplus \cdots,
$$

Recall that in Section 4 , we showed that $c_{1}(\Sigma) \in H^{1}\left(\Sigma, \Omega_{\Sigma}^{1, \mathrm{cl}}\right)$ and $\left(c_{1}(\mathcal{E})-\right.$ $\left.c_{1}(T X)\right) \in H^{1}\left(X, \Omega_{X}^{1, c l}\right)$. Hence, the two physical anomalies $\operatorname{ch}_{2}(\mathcal{E})-\operatorname{ch}_{2}(T X)$ 
and $\frac{1}{2} c_{1}(\Sigma)\left(c_{1}(\mathcal{E})-c_{1}(T X)\right)$ take values in the first and second term on the RHS of (5.43), respectively. Note that the terms on the RHS of (5.43) must independently vanish for $H^{2}\left(X \times \Sigma, \Omega_{X \times \Sigma}^{2, \text { cl }}\right)$ to be zero. In other words, we have obtained a consistent, alternative interpretation of the physical anomalies which arise due to a non-triviality of the determinant line bundles (associated with the Dirac operators of the underlying sigma model) over the space of gauge-inequivalent connections, purely in terms of an obstruction to the gluing of sheaves of chiral algebras.

By extending the arguments surrounding (5.35) to the present context, we find that for a vanishing anomaly, apart from the geometrical moduli encoded in the holomorphic and complex structures of the bundle $\mathcal{E} \rightarrow X$, the moduli of the globally defined sheaf of chiral algebras on $\Sigma$, with target space $\mathcal{E} \rightarrow X$, must be parameterized by $H^{1}\left(X \times \Sigma, \Omega_{X \times \Sigma}^{2, \mathrm{cl}}\right)$.

\subsection{The conformal anomaly}

In this section, we will demonstrate an application of the rather abstract discussion thus far. In the process, we will be able to provide a physical interpretation of a computed mathematical result and vice-versa.

From equation (3.1), we see that the holomorphic stress tensor $T(z) \sim T_{z z}$ of the twisted heterotic sigma model lacks the $\psi^{\bar{i}}$ fields. ${ }^{22}$ In other words, it is an operator with $q_{\mathrm{R}}=0$. Hence, from the $\bar{Q}_{+}$-Cech cohomology dictionary established in Section 5.3, if $T(z)$ is to be non-trivial in $\bar{Q}_{+}$-cohomology, such that the sigma model and its chiral algebra are conformally invariant, it will be given by an element of $H^{0}(X, \widehat{\mathcal{A}})$, that is, a global section of the sheaf of chiral algebras $\widehat{\mathcal{A}}$. Recall that the local sections of $\widehat{\mathcal{A}}$ are furnished by the physical operators in the chiral algebra of the free (linear) $b c-\beta \gamma$ system. Since the free (linear) $b c-\beta \gamma$ system describes a local version of the underlying twisted heterotic sigma model, one can write the local holomorphic stress tensor of the sigma model as the local holomorphic stress tensor of the free (linear) $b c-\beta \gamma$ system, which in turn is given by

$$
\mathcal{T}(z)=-: \beta_{i} \partial_{z} \gamma^{i}:-: b_{a} \partial_{z} c^{a}: .
$$

(see Section 5.4). Under an automorphism of the $b c-\beta \gamma$ system, $\mathcal{T}(z)$ will become

$$
\widetilde{\mathcal{T}}(z)=-: \tilde{\beta}_{i} \partial_{z} \tilde{\gamma}^{i}:-: \tilde{b}_{a} \partial_{z} \tilde{c}^{a}:
$$

\footnotetext{
${ }^{22}$ Recall that this is also true in the quantum theory as the classical expression for $T(z)$ does not receive any perturbative corrections up to 1-loop.
} 
where the fields $\tilde{\beta}, \tilde{\gamma}, \tilde{b}$, and $\tilde{c}$ are defined in the automorphism relations of (5.22)-(5.25). It is clear that on an overlap $U_{a} \cap U_{b}$ in $X, \mathcal{T}(z)$ will be regular in $U_{a}$ while $\widetilde{\mathcal{T}}(z)$ will be regular in $U_{b}$. Note that both $\mathcal{T}(z)$ and $\widetilde{\mathcal{T}}(z)$ are at least local sections of $\widehat{\mathcal{A}}$. And if there is no obstruction to $\mathcal{T}(z)$ or $\widetilde{\mathcal{T}}(z)$ being a global section of $\widehat{\mathcal{A}}$, it will mean that $T(z)$ is non-trivial in $\bar{Q}_{+}$-cohomology, i.e., $T(z) \neq\left\{\bar{Q}_{+}, \ldots\right\}$ and $\left[\bar{Q}_{+}, T(z)\right]=0$, and the sigma model will be conformally invariant. For $\mathcal{T}(z)$ or $\tilde{\mathcal{T}}(z)$ to be a global section of $\widehat{\mathcal{A}}$, it must be true that $\mathcal{T}(z)=\widetilde{\mathcal{T}}(z)$ on any overlap $U_{a} \cap U_{b}$ in $X$. Let us examine this further by considering an example.

For ease of illustration, we shall consider an example whereby $\operatorname{dim}_{\mathbb{C}} X=$ $\operatorname{rank}(\mathcal{E})=1$, say $\mathcal{E}$ is a certain $U(1)$ line bundle over $X=\mathbb{C P}^{1}$. In order for us to consider an underlying sigma model that is physically consistent (whereby one can at least define a sheaf of chiral algebras globally over $\mathbb{C P}^{1}$ ), we require that $\mathcal{E}$ be chosen such that $\operatorname{ch}_{2}(\mathcal{E})=\operatorname{ch}_{2}\left(T \mathbb{C P}^{1}\right)$. However, we do not necessarily require that $c_{1}(\mathcal{E})=c_{1}\left(T \mathbb{C P}^{1}\right)$ (and why this is so would be clear momentarily). Since $\mathbb{C P}^{1}$ can be considered as the complex $\gamma$-plane plus a point at infinity, we can cover it with two open sets, $U_{1}$ and $U_{2}$, where $U_{1}$ is the complex $\gamma$-plane, and $U_{2}$ is the complex $\tilde{\gamma}$-plane, such that $\tilde{\gamma}=1 / \gamma$. And since $\mathcal{E}$ is a $U(1)$ line bundle, the transition function $A$ in (5.22)-(5.25) will be given by $\mathrm{e}^{i \theta(\gamma)}$, where $\theta(\gamma)$ is some real, holomorphic function of $\gamma$. By substituting the definitions of $\tilde{\beta}, \tilde{\gamma}, \tilde{b}$, and $\tilde{c}$ from (5.22)-(5.25) into $\tilde{\mathcal{T}}(z)$, we compute that ${ }^{23}$

$$
\widetilde{\mathcal{T}}(z)-\mathcal{T}(z)=\partial_{z}\left(\frac{\partial_{z} \gamma}{\gamma}\right)+\cdots
$$

where "..." are terms involving the fields $b, c$, and the function $\theta(\gamma)$. Note that, in general, there is no sensible way to remove the terms on the RHS of (5.46) through a consistent redefinition of $\mathcal{T}(z)$ and $\widetilde{\mathcal{T}}(z)$ (such that $\mathcal{T}(z)$ and $\tilde{\mathcal{T}}(z)$ continue to be invariant under the symmetries of the terms on the RHS of (5.46) and have the correct OPEs, as stress tensors, with the elementary fields $\beta, \gamma, b$, and $c)$. Hence, we find that neither $\mathcal{T}(z)$ nor $\tilde{\mathcal{T}}(z)$ can be a global section of $\widehat{\mathcal{A}}$, i.e., $\mathcal{T}(z), \widetilde{\mathcal{T}}(z) \notin H^{0}\left(\mathbb{C P}^{1}, \widehat{\mathcal{A}}\right)$. In other words, $T(z)$ is not in the $\bar{Q}_{+}$-cohomology of the sigma model; there is a conformal anomaly. This is consistent with an earlier observation made in Section 3.1 via equation $(3.6)$, where $\left[\bar{Q}_{+}, T_{z z}\right] \neq 0$ in general but

$$
\left[\bar{Q}_{+}, T_{z z}\right]=\partial_{z}\left(R_{i \bar{j}} \partial_{z} \phi^{i} \psi^{\bar{j}}\right)+\cdots
$$

\footnotetext{
${ }^{23}$ Note that in our computation, we have conveniently chosen the arbitrary, local $(1,0)$ form $B(\gamma) d \gamma$ on $\mathbb{C P}^{1}$ (associated with the current $J_{B}$ of Section 5.5) to be one with $B(\gamma)=2 \gamma$.
} 
Note that since $\bar{Q}_{+}$generates the BRST symmetry (i.e., an automorphism) of the twisted heterotic sigma model via the field transformations (2.11), (5.46) will be an analogue in the Cech cohomology of relation (5.47) (as briefly mentioned in footnote 5 of Section 3.1). In fact, $R_{i \bar{j}} \partial_{z} \phi^{i} \psi^{\bar{j}}$ can be interpreted as the counterpart of the term $\partial_{z} \gamma / \gamma$ in conventional physics notation as follows. Apart from an obvious comparison of (5.47) and (5.46), note that $\partial_{z} \gamma / \gamma=-\partial_{z} \tilde{\gamma} / \tilde{\gamma}$, i.e., $\partial_{z} \gamma / \gamma$ is a holomorphic operator over $U_{1} \cap$ $U_{2}$. Moreover, it cannot be expressed as a difference between an operator that is holomorphic in $U_{1}$ and an operator that is holomorphic in $U_{2}$. Thus, it is a dimension 1 class in the first Cech cohomology group $H^{1}\left(\mathbb{C P}^{1}, \widehat{\mathcal{A}}\right)$. Hence, from our $\bar{Q}_{+}$-Cech cohomology dictionary, $\partial_{z} \gamma / \gamma$ will correspond to a dimension 1 operator in the $\bar{Q}_{+}$-cohomology of the sigma model with $q_{R}=1$, namely $R_{i \bar{j}} \partial_{z} \phi^{i} \psi^{\bar{j}}$ (which indeed takes the correct form of a $\bar{Q}_{+}$-invariant, dimension $(1,0)$ operator with $q_{R}=1$ as discussed in Section 5.1). Since the Ricci tensor $R_{i \bar{j}}$ is proportional to the 1-loop beta-function of the sigma model, this correspondence allows one to interpret the 1-loop beta-function purely in terms of holomorphic data.

One can certainly consider other higher dimensional examples in a similar fashion. In fact, it can be shown mathematically that $\widetilde{\mathcal{T}}(z) \neq \mathcal{T}(z)$ for any $X$ and $\mathcal{E}$ if $\left[c_{1}(\mathcal{E})-c_{1}(T X)\right] \neq 0[3,5]$. One can indeed see that $\left[c_{1}(\mathcal{E})-c_{1}(T X)\right]$ characterizes a conformal anomaly of the twisted heterotic sigma model as follows. Recall from Section 3.1 that the RHS of (5.47) captures the violation in the conformal structure of the sigma model by the 1-loop beta-function. It will vanish if $X$ is a Ricci-flat manifold and if the curvature of the bundle $\mathcal{E}$ obeys the Donaldson-Uhlenbeck-Yau equation. Both these conditions can be trivially satisfied if $c_{1}(T X)=c_{1}(\mathcal{E})=0$, which then implies that $\left[c_{1}(\mathcal{E})-c_{1}(T X)\right]=0$.

Thus, the obstruction to a globally defined $T(z)$ operator, characterized by a non-vanishing cohomology class $\left[c_{1}(\mathcal{E})-c_{1}(T X)\right]$, translates to a lack of invariance under arbitrary, holomorphic reparameterizations on the worldsheet $\Sigma$ of the $\bar{Q}_{+}$-cohomology of the underlying twisted heterotic sigma model.

\section{The half-twisted $(2,2)$ model and the CDR complex}

In this section, we shall consider a specific situation in which the anomalies discussed in Section 5.6 automatically vanish, thus enabling us to consider, in Section 7, other interesting and physically consistent applications of the sheaf of chiral algebras that we have been studying so far. In the process, we will be able to furnish a purely physical interpretation of the 
sheaf of CDOs constructed by Malikov et al. in [1,2], known as the CDR complex. The results in this section also serve as a generalization and an alternative verification of an earlier observation made by Kapustin [11] on the connection between a half-twisted $(2,2)$ sigma model on a Calabi-Yau manifold and the sheaf of CDR complex, and Frenkel and Losev's computation in [13]. In addition, via the physical definition of the elliptic genus as a specialization of the genus 1 partition function, and the CFT state-operator correspondence in the Calabi-Yau case, we can also make contact with the mathematical definition of the elliptic genus found in [8-10]. This further suggests that the local operators in the $\bar{Q}_{+}$-cohomology, which furnish a chiral algebra $\mathcal{A}$, can indeed be represented by the appropriate classes in the Cech cohomology group generated by the relevant sheaf of CDOs or vertex superalgebras.

\subsection{The $(2,2)$ locus and the half-twisted $(2,2)$ sigma model}

The $(2,2)$ locus is defined as the set in the moduli space of holomorphic vector bundles $\mathcal{E}$ whereby $\mathcal{E}=T X$. Thus, notice that on the $(2,2)$ locus, both the anomalies of the underlying twisted heterotic sigma model, namely $\operatorname{ch}_{2}(\mathcal{E})-\operatorname{ch}_{2}(T X)$ and $\frac{1}{2} c_{1}(\Sigma)\left(c_{1}(\mathcal{E})-c_{1}(T X)\right)$, vanish independently for any $X$. Moreover, $\operatorname{rank}(\mathcal{E})=r=\operatorname{dim}_{\mathbb{C}} X$, and the physical constraint $\Lambda^{r} \mathcal{E}^{\vee} \cong K_{X}$ is trivially satisfied.

Since $\mathcal{E}=T X$ at the $(2,2)$ locus, one can make the following field replacements: $\lambda_{z a} \rightarrow g_{i \bar{j}} \psi_{z}^{\bar{j}}, \lambda^{a} \rightarrow \psi^{i}, A(\phi) \rightarrow \Gamma(\phi)$, and $F(\phi) \rightarrow R(\phi)$, where $A(\phi)$ and $F(\phi)$ are the connection and field strength of the gauge bundle $\mathcal{E}$, while $\Gamma(\phi)$ and $R(\phi)$ are the affine connection and Riemann curvature of $X$. In making these replacements in $S_{\text {pert }}$ of (2.15), we find that the action of the underlying twisted sigma model at the $(2,2)$ locus will be given by

$S_{(2,2)}=\int_{\Sigma}\left|d^{2} z\right|\left(g_{i \bar{j}} \partial_{z} \phi^{\bar{j}} \partial_{\bar{z}} \phi^{i}+g_{i \bar{j}} \psi_{\bar{z}}^{i} D_{z} \psi^{\bar{j}}+g_{i \bar{j}} \psi_{z}^{\bar{j}} D_{\bar{z}} \psi^{i}-R_{i \bar{k} j \bar{l}} \psi_{\bar{z}}^{i} \psi_{z}^{\bar{k}} \psi^{j} \psi^{\bar{l}}\right)$,

where $i, j, k, l=1,2, \ldots, \operatorname{dim}_{\mathbb{C}} X . R_{i \bar{k} j \bar{l}}$ is the curvature tensor with respect to the Levi-Civita connection $\Gamma^{i}{ }_{l j}=g^{i \bar{k}} \partial_{l} g_{j \bar{k}}$, and the covariant derivatives with respect to the connection induced on the worldsheet are given by

$$
D_{z} \psi^{\bar{j}}=\partial_{z} \psi^{\bar{j}}+\Gamma^{\bar{j}_{\bar{i}}} \partial_{z} \phi^{\bar{i}} \psi^{\bar{k}}, \quad D_{\bar{z}} \psi^{i}=\partial_{\bar{z}} \psi^{i}+\Gamma_{j k}^{i} \partial_{\bar{z}} \phi^{j} \psi^{k}
$$

Notice that $S_{(2,2)}$ just coincides with the (perturbative) action of Witten's topological A-model defined in [27]. ${ }^{24}$

\footnotetext{
${ }^{24}$ The action $S_{(2,2)}$ just differs from the A-model action in [27] by a term $\int_{\Sigma} \Phi^{*}(K)$, where $K$ is the Kähler $(1,1)$-form on $X$. This term is irrelevant in perturbation theory where one considers trivial maps of degree zero as explained in Section 2.2.
} 
As a relevant digression, note that in Witten's topological A-model, the BRST-charge operator that defines the BRST cohomology is given by $Q_{\mathrm{BRST}}=Q_{\mathrm{L}}+Q_{\mathrm{R}}$, where $Q_{\mathrm{L}}=Q_{-}$is an additional left-moving (scalar) supercharge from the $(2,2)$ superalgebra. However, the A-model has a greatly enriched variant in which one ignores $Q_{\mathrm{L}}$ and considers $Q_{\mathrm{R}}$ as the BRST operator [17]. This variant is also known as the half-twisted $(2,2)$ sigma model [27]. Since the corresponding cohomology is now defined with respect to a single, right-moving, scalar supercharge $Q_{\mathrm{R}}$, its classes need not be restricted to dimension $(0,0)$ operators (which correspond to ground states). In fact, the physical operators will have dimension $(n, 0)$, where $n \geq 0$. (The arguments supporting this statement are similar to those in Section 3.1 concerning the scaling dimensions of local operators in the twisted heterotic sigma model which are closed with respect to a single, right-moving supercharge $\bar{Q}_{+}$.) Hence, in contrast to the A-model, the BRST spectrum of physical operators and states in the half-twisted model is infinitedimensional. A specialization of its genus 1 partition function, also known as the elliptic genus of $X$, is given by the index of the $Q_{\mathrm{R}}$ operator. The halftwisted model is not a topological field theory - the stress tensor derived from its action is exact with respect to the combination $Q_{\mathrm{L}}+Q_{\mathrm{R}}$, but not $Q_{\mathrm{R}}$ alone. In fact, the correlation functions of the local, $Q_{\mathrm{R}}$-invariant holomorphic operators that furnish its chiral algebra, although invariant under arbitrary scalings of the worldsheet, are not independent of its other geometrical properties; the correlation functions vary holomorphically with the complex structure on the worldsheet (as is familiar for chiral algebras). Moreover, since the (holomorphic) stress tensor does not vanish in $Q_{\mathrm{R}^{-}}$ cohomology, the chiral algebra of the half-twisted model will be invariant under holomorphic reparameterizations of the coordinates on the worldsheet.

Now, recall that our current objective is to study, in terms of the relevant sheaf of CDOs and its Cech cohomology, the chiral algebra spanned by the $\bar{Q}_{+}$-cohomology classes of the sigma model with action $S_{(2,2)}$. From the supersymmetry variations in $(2.11)$, and the action $S_{\text {pert }}$ in $(2.15)$, we find that the supercurrent of the scalar supercharge $\bar{Q}_{+}$is given by $\bar{Q}_{+}(\bar{z})=$ $g_{i \bar{j}} \psi^{\bar{j}} \partial_{\bar{z}} \phi^{i}$. As will be shown shortly, the supercurrent of the scalar supercharge $Q_{\mathrm{R}}$ of the half-twisted A-model is also given by $Q_{\mathrm{R}}(\bar{z})=g_{i \bar{j}} \psi^{\bar{j}} \partial_{\bar{z}} \phi^{i}$. Thus, the BRST operators $Q_{\mathrm{R}}$ and $\bar{Q}_{+}$coincide. Hence, we find that by studying the twisted heterotic sigma model at the $(2,2)$ locus in terms of a sheaf of CDOs or chiral algebras, we are effectively studying the half-twisted $(2,2)$ model in the same spirit. We shall henceforth refer to the model with action $S_{(2,2)}$ and its corresponding chiral algebra of local operators in the $\bar{Q}_{+}$-cohomology as the half-twisted $(2,2)$ sigma model.

Based on the discussion in Section 3.2, a moduli for the chiral algebra can be incorporated into the half-twisted model by introducing a non-Kähler 
deformation of $X$ via the addition of the $\mathcal{H}$-flux term (3.9) to the action $S_{(2,2)}$. As argued in Section 5.6, the moduli of the corresponding, globally defined sheaf of CDOs on $X$ can then be represented by a class in $H^{1}\left(X, \Omega_{X}^{2, \mathrm{cl}}\right)$.

\subsubsection{A holomorphic twisted $N=2$ superconformal algebra}

We shall now examine the holomorphic structure of the half-twisted $(2,2)$ model with action $S_{(2,2)}$. The reason for doing so is that some of its nontrivial aspects can be captured by the characteristics of the sheaf of chiral algebras describing the sigma model on $X$. Moreover, similar to what we had seen in Section 5.7, one can also derive an interpretation of these nontrivial aspects purely in terms of mathematical data and vice-versa. We will demonstrate these claims shortly when we consider an example in Section 7.1. To this end, let us first discuss the classical symmetries of $S_{(2,2)}$ and their corresponding conserved currents and tensors.

First, note that $S_{(2,2)}$ has a left- and right-moving ghost number symmetry, whereby the left-moving fermionic fields transform as $\psi^{i} \rightarrow \mathrm{e}^{i \alpha} \psi^{i}$ and $\psi_{z}^{\bar{i}} \rightarrow \mathrm{e}^{-i \alpha} \psi_{z}^{\bar{i}}$ and the right-moving fermionic fields transform as $\psi^{\bar{i}} \rightarrow \mathrm{e}^{i \alpha} \psi^{\bar{i}}$ and $\psi_{\bar{z}}^{i} \rightarrow \mathrm{e}^{-i \alpha} \psi_{\bar{z}}^{i}$, where $\alpha$ is real. In other words, the fields $\psi^{i}, \psi_{z}^{\bar{i}}, \psi^{\bar{i}}$, and $\psi_{\bar{z}}^{i}$ can be assigned the $\left(g_{\mathrm{L}}, g_{\mathrm{R}}\right)$ left-right ghost numbers $(1,0),(-1,0)$, $(0,1)$ and $(0,-1)$, respectively. The infinitesimal version of this symmetry transformation of the left-moving fermi fields read (after absorbing some trivial constants)

$$
\delta \psi^{i}=\psi^{i}, \quad \delta \psi_{z}^{\bar{i}}=-\psi_{z}^{\bar{i}}
$$

while those of the right-moving fermi fields read

$$
\delta \psi^{\bar{i}}=\psi^{\bar{i}}, \quad \delta \psi_{\bar{z}}^{i}=-\psi_{\bar{z}}^{i}
$$

The conserved holomorphic (i.e., left-moving) current associated with the transformation (6.3) will then be given by

$$
J(z)=g_{i j} \psi_{z}^{\bar{j}} \psi^{i} .
$$

$J(z)$ is clearly a dimension 1 bosonic current. (There is also an anti-holomorphic conserved current associated with the right-moving ghost symmetry. However, it will not be relevant in our discussion.) Secondly, note that $S_{(2,2)}$ is also invariant under the following field transformations:

$$
\begin{aligned}
\delta \phi^{i} & =\psi^{i}, \quad \delta \phi^{\bar{i}}=0, \\
\delta \psi_{z}^{\bar{i}} & =-\partial_{z} \phi^{\bar{i}}, \quad \delta \psi_{\bar{z}}^{i}=-\Gamma_{j k}^{i} \psi^{j} \psi_{\bar{z}}^{k} \\
\delta \psi^{i} & =0, \quad \delta \psi^{\bar{i}}=0 .
\end{aligned}
$$


The conserved, dimension 1 fermionic current in this case will be given by

$$
Q(z)=g_{i \bar{j}} \psi^{i} \partial_{z} \phi^{\bar{j}}
$$

In fact, the following field transformations

$$
\begin{aligned}
\delta \phi^{\bar{i}} & =\psi^{\bar{i}}, \quad \delta \phi^{i}=0, \\
\delta \psi_{\bar{z}}^{i} & =-\partial_{\bar{z}} \phi^{i}, \quad \delta \psi_{z}^{\bar{i}}=-\Gamma^{\bar{i}}{ }_{\bar{j} \bar{k}} \psi^{\bar{j}} \psi_{z}^{\bar{k}}, \\
\delta \psi^{i} & =0, \quad \delta \psi^{\bar{i}}=0,
\end{aligned}
$$

will also leave $S_{(2,2)}$ invariant. The corresponding, conserved generator of the transformation (6.8) is given by $Q_{\mathrm{R}}$, the right-moving supercharge of the original A-model, where the supercurrent is $Q_{\mathrm{R}}(\bar{z})=g_{i \bar{j}} \psi^{\bar{j}} \partial_{\bar{z}} \phi^{i}$. However, note that $Q_{\mathrm{R}}(\bar{z})$ does not contribute to the holomorphic structure of the halftwisted model, as it is an anti-holomorphic (i.e., right-moving) dimension 1 supercurrent.

Another conserved quantity that will be relevant to our present discussion is the dimension 2 holomorphic stress tensor $T(z)=-T_{z z}$ associated with the symmetry under holomorphic reparameterizations of the coordinates on the worldsheet. It is given by

$$
T(z)=-g_{i \bar{j}} \partial_{z} \phi^{i} \partial_{z} \phi^{\bar{j}}-g_{i \bar{j}} \psi_{z}^{\bar{j}} D_{z} \psi^{i} .
$$

Observe that one can also derive a fourth conserved current $G(z)$ by noting that $T(z)=\{Q, G(z)\}=\delta G(z)$, the variation of $G(z)$ under the field transformations (6.6), where

$$
G(z)=g_{i \bar{j}} \psi_{z}^{\bar{j}} \partial_{z} \phi^{i}
$$

(Note that the half-twisted model remains a non-topological model $-T(z)$ is $Q$-exact but not $Q_{\mathrm{R}}$-exact.) Clearly, $G(z)$ is a dimension 2 fermionic current. Note that since these currents possess only holomorphic scaling dimensions, their respective spins will also be given by their dimensions.

One can verify that $J(z), Q(z), T(z)$ and $G(z)$ are all invariant under the field transformations of (6.8). In other words, they are $Q_{\mathrm{R}}$-invariant operators which therefore commute or anti-commute with $Q_{\mathrm{R}}$. As noted earlier, $Q_{\mathrm{R}}$ coincides with $\bar{Q}_{+}$. Hence, we find that $J(z), Q(z), T(z)$, and $G(z)$ are $\bar{Q}_{+}$-closed operators in the $\bar{Q}_{+}$-cohomology at the classical level. Also note at this point that if $\mathcal{O}$ and $\mathcal{O}^{\prime}$ are $\bar{Q}_{+}$-closed operators in the $\bar{Q}_{+}$-cohomology, i.e., $\left\{\bar{Q}_{+}, \mathcal{O}\right\}=\left\{\bar{Q}_{+}, \mathcal{O}^{\prime}\right\}=0$, then $\left\{\bar{Q}_{+}, \mathcal{O O}^{\prime}\right\}=0$. Moreover, if $\left\{\bar{Q}_{+}, \mathcal{O}\right\}=0$, then $\mathcal{O}\left\{\bar{Q}_{+}, W\right\}=\left\{\bar{Q}_{+}, \mathcal{O} W\right\}$ for any operator $W$. These two statements mean that the cohomology classes of operators that commute with $\bar{Q}_{+}$form a closed (and well-defined) algebra under operator products. One can indeed show that $J(z), Q(z), T(z)$, and $G(z)$ form a 
complete multiplet which generates a closed, holomorphic, twisted $N=2$ superconformal algebra with the following OPE relations [28]:

$$
\begin{gathered}
T(z) T(w) \sim \frac{2 T(w)}{(z-w)^{2}}+\frac{\partial T(w)}{z-w}, \\
J(z) J(w) \sim \frac{d}{(z-w)^{2}} ; \quad T(z) J(w) \sim-\frac{d}{(z-w)^{3}}+\frac{J(w)}{(z-w)^{2}}+\frac{\partial J(w)}{z-w}, \\
G(z) G(w) \sim 0 ; \quad T(z) G(w) \sim \frac{2 G(w)}{(z-w)^{2}}+\frac{\partial G(w)}{z-w} ; \quad J(z) G(w) \sim-\frac{G(w)}{z-w}, \\
(6.10 c) \\
Q(z) Q(w) \sim 0 ; \quad T(z) Q(w) \sim \frac{Q(w)}{(z-w)^{2}}+\frac{\partial Q(w)}{z-w} ; \quad J(z) Q(w) \sim \frac{Q(w)}{z-w}, \\
(6.10 d) \\
Q(z) G(w) \sim \frac{d}{(z-w)^{3}}+\frac{J(w)}{(z-w)^{2}}+\frac{T(w)}{z-w},
\end{gathered}
$$

where $d=\operatorname{dim}_{\mathbb{C}} X$. This structure is also known as a structure of a topological vertex algebra of rank $d$ in the mathematical literature [1]. Thus, we see that $G(z)$ is a (worldsheet) superpartner of $T(z)$ under the supersymmetry generated by the charge $Q$ of the supercurrent $Q(z)$. In addition, we also find from the OPEs that $[Q, J(z)]=-Q(z)$, i.e., $J(z)$ is a (worldsheet) superpartner of $Q(z)$. These observations will be relevant momentarily. Also notice that the central charge in the stress tensor OPE (6.10a) is zero. This means that the Weyl anomaly vanishes and that the trace of the stress tensor is trivial in $\bar{Q}_{+}$-cohomology at the quantum level. This simply reflects the invariance of the correlation functions under scalings of the worldsheet as noted earlier.

The classical, holomorphic, OPE algebra of the half-twisted model in (6.10) may or may not persist in the quantum theory. In fact, in a "massive" model where the first Chern class $c_{1}(X)$ is non-vanishing, the global $U(1)$ symmetry associated with $J(z)$ will be broken; likewise for the symmetry associated with its superpartner $Q(z)$. Hence, $J(z)$ and $Q(z)$ will cease to remain in the $\bar{Q}_{+}$-cohomology at the quantum level. However, the symmetries associated with $T(z)$ and $G(z)$ are exact in quantum perturbation theory, and these operators will remain in the $\bar{Q}_{+}$-cohomology at the quantum level. This is consistent with the fact that the conformal anomaly discussed in Section 5.7 vanishes for $\mathcal{E}=T X$. Hence, for $c_{1}(X) \neq 0$, we have in some sense a reduction from an $N=2$ to an $N=1$ algebra. We will examine this more closely from a different point of view when we consider an example in Section 7.1, where we describe the half-twisted model in terms of a sheaf of CDOs. Once again, we will be able to obtain a purely mathematical 
interpretation of the above physical observations. In particular, we can interpret the non-vanishing beta-function solely in terms of holomorphic data.

\subsection{2 $\overline{\mathrm{Q}}_{+}$-cohomology classes of local operators}

We shall now discuss the $\bar{Q}_{+}$-cohomology of local operators which furnish a holomorphic chiral algebra $\mathcal{A}$ of the half-twisted sigma model. Note that we can describe the structure of the chiral operators in the half-twisted model by specializing the arguments made in Section 5.1 to the case where $\mathcal{E}=T X$. This can be achieved by making the field replacements $\lambda_{z a} \rightarrow$ $g_{i \bar{j}} \psi_{z}^{\bar{j}}=\psi_{z i}$ (where $\psi_{z i} \in K \otimes \Phi^{*}\left(T^{*} X\right)$ ) and $\lambda^{a} \rightarrow \psi^{i}$. In general, we find that a local operator $\mathcal{F}$ in the $\bar{Q}_{+}$-cohomology of the half-twisted model will be given by $\mathcal{F}\left(\phi^{i}, \partial_{z} \phi^{i}, \partial_{z}^{2} \phi^{i}, \ldots ; \phi^{\bar{i}}, \partial_{z} \phi^{\bar{i}}, \partial_{z}^{2} \phi^{\bar{i}}, \ldots ; \psi_{z i}, \partial_{z} \psi_{z i}, \partial_{z}^{2} \psi_{z i} \ldots ; \psi^{i}\right.$, $\left.\partial_{z} \psi^{i}, \partial_{z}^{2} \psi^{i} \ldots ; \psi^{\bar{i}}\right)$. If $\mathcal{F}$ is homogeneous of degree $k$ in $\psi^{\bar{i}}$, then it has ghost number $\left(g_{\mathrm{L}}, g_{\mathrm{R}}\right)=(p, k)$, where $p$ is determined by the net number of $\psi^{i}$ over $\psi_{z i}$ fields (and/or of their corresponding derivatives) in $\mathcal{F}$. An operator $\mathcal{F}\left(\phi^{i}, \partial_{z} \phi^{i}, \ldots ; \phi^{\bar{i}}, \partial_{z} \phi^{\bar{i}}, \ldots ; \psi_{z i}, \partial_{z} \psi_{z i}, \ldots ; \psi^{i}, \partial_{z} \psi^{i}, \ldots ; \psi^{\bar{i}}\right)$ with $q_{\mathrm{R}}=k$ can be interpreted as a $(0, k)$-form on $X$ with values in a certain tensor product bundle. For example, a dimension $(0,0)$ operator will generally take the form $\mathcal{F}\left(\phi^{i}, \phi^{\bar{i}} ; \psi^{j} ; \psi^{\bar{j}}\right)=f_{i_{1}, \ldots, i_{q} ; \bar{j}_{1}, \ldots, \bar{j}_{k}}\left(\phi^{k}, \phi^{\bar{k}}\right) \psi^{i_{1}} \cdots \psi^{i_{q}} \psi^{\bar{j}_{i}} \cdots \psi^{\bar{j}_{k}}$. Such an operator will correspond to an ordinary $(q, k)$-form $f_{i_{1}, \ldots, i_{q} \bar{j}_{1}, \ldots, \bar{j}_{k}}\left(\phi^{i}, \phi^{\bar{i}}\right) d \phi^{i_{1}} \cdots d \phi^{i_{q}}$ $d \phi^{\bar{j}_{1}} \cdots d \phi^{\bar{j}_{k}}$ on $X$. For dimension (1,0) operators, one will have a few cases. For example, we can have an operator $\mathcal{F}\left(\phi^{l}, \phi^{\bar{l}} ; \partial_{z} \phi^{\bar{i}}, \psi^{i} ; \psi^{\bar{j}}\right)=f^{j}{ }_{i_{1}, \ldots, i_{q} ; \bar{j}_{1}, \ldots, \bar{j}_{k}}$ $\left(\phi^{l}, \phi^{\bar{l}}\right) g_{j \bar{i}} \partial_{z} \phi^{\bar{i}} \psi^{i_{1}} \cdots \psi^{i_{q}} \psi^{\bar{j}_{i}} \cdots \psi^{\bar{j}_{k}}$ that is linear in $\partial_{z} \phi^{\bar{i}}$. Such an operator will correspond to a $(q, k)$-form on $X$ with values in the holomorphic tangent bundle $T X$. We can also have an operator $\mathcal{F}\left(\phi^{l}, \phi^{\bar{l}} ; \partial_{z} \phi^{i}, \psi^{i} ; \psi^{\bar{j}}\right)=$ $f_{i_{1}, \ldots, i_{q} ; \bar{j}_{1}, \ldots, \bar{j}_{k}}^{\bar{j}}\left(\phi^{l}, \phi^{\bar{l}}\right) g_{\bar{j} i} \partial_{z} \phi^{i} \psi^{i_{1}} \ldots \psi^{i_{q}} \psi^{\bar{j}_{1}} \ldots \psi^{\bar{j}_{k}}$ that is linear in $\partial_{z} \phi^{i}$. Such an operator will correspond to a $(q, k)$-form on $X$ with values in the bundle $\overline{T X}$. In a similar fashion, for any integer $n>0$, the operators of dimension $(n, 0)$ and charge $q_{\mathrm{R}}=k$ can be interpreted as $(0, k)$-forms with values in a certain tensor product bundle over $X$. This structure persists in quantum perturbation theory, but there may be perturbative corrections to the complex structure of the bundle.

Based on the discussion in Section 5.1, the action of $\bar{Q}_{+}$can be described as follows. First, at the classical level, $\bar{Q}_{+}$does not act as $\bar{\partial}=d \phi^{\bar{i}} \partial / \partial \phi^{\bar{i}}$ on a general operator $\mathcal{F}$ that contains the derivatives $\partial_{z}^{m} \phi^{\bar{i}}$ for $m>0$. However, it will do so on dimension $(0,0)$ operators, in the absence of perturbative corrections. Secondly, if $X$ is flat, $\bar{Q}_{+}$will act as the $\bar{\partial}$ operator on any $\mathcal{F}$ at the classical level. This is because the equation of motion $D_{z} \psi^{\bar{i}}=0$ ensures that the action of $\bar{Q}_{+}$on derivatives $\partial_{z}^{m} \phi^{\bar{i}}$ for $m>0$ can be ignored. 
Moreover, since $\delta \psi_{z i}=0$ for a flat metric, one can also ignore the action of $\bar{Q}_{+}$on the $\psi_{z i}$ fields and their derivatives $\partial_{z}^{m} \psi_{z i}$ with $m>0$. At the quantum level, for $X$, a flat manifold, $\bar{Q}_{+}$may receive perturbative corrections from $\bar{\partial}$-cohomology classes that are constructed locally from the fields appearing in the action such as the class in $H^{1}\left(X, \Omega_{X}^{2, \text { cl }}\right)$.

\subsubsection{A topological chiral ring}

From the arguments in Section 5.2, we learn that the $\bar{Q}_{+}$-invariant ground (i.e., dimension $(0,0)$ ) operators $\widetilde{\mathcal{F}}$ define a topological chiral ring via their OPE

$$
\widetilde{\mathcal{F}}_{a} \widetilde{\mathcal{F}}_{b}=\sum_{q_{c}=q_{a}+q_{b}} C_{a b c} \widetilde{\mathcal{F}}_{c}
$$

where $C_{a b c}$ are structure constants, anti-symmetric in their indices, and $q_{a}$ and $q_{b}$ represent the $\left(g_{\mathrm{L}}, g_{\mathrm{R}}\right)$ ghost number of $\widetilde{\mathcal{F}}_{a}$ and $\widetilde{\mathcal{F}}_{b}$, respectively. The ring is effectively $\mathbb{Z}_{2} \times \mathbb{Z}_{2}$ graded in the absence of non-perturbative worldsheet instantons. At the classical level (in the absence of perturbative corrections), $\bar{Q}_{+}$acts as $Q_{\mathrm{cl}}=\bar{\partial}$ on any dimension $(0,0)$ opera-

tor $\widetilde{\mathcal{F}}$. Since an arbitrary dimension $(0,0)$ operator $\widetilde{\mathcal{F}}_{d}$ with $\left(g_{\mathrm{L}}, g_{\mathrm{R}}\right)=$ $(q, k)$ corresponds to an ordinary $(q, k)$-form $f_{i_{1}, \ldots, i_{q}, \bar{j}_{1}, \ldots, \bar{j}_{k}}\left(\phi^{i}, \phi^{\bar{i}}\right) d \phi^{i_{1}} \wedge \cdots \wedge$ $d \phi^{i_{q}} \wedge d \phi^{\bar{j}_{1}} \wedge \cdots \wedge d \phi^{\bar{j}_{k}}$ on $X$, the classical ring is just the graded Dolbeault cohomology ring $H_{\bar{\partial}}^{*, *}(X)$. Alternatively, via the Cech-Dolbeault isomorphism in ordinary differential geometry, the classical ring can also be interpreted as the graded Cech cohomology ring $H^{*}\left(X, \Lambda^{*} T X^{\vee}\right)$, where $T X^{\vee}$ is the holomorphic cotangent bundle on $X$. The operators $\widetilde{\mathcal{F}}$ will either be non-Grassmannian or Grassmannian, obeying either commutators or anticommutators, depending on whether they contain an even or odd number of fermionic $\psi$ fields.

\subsection{Sheaf of CDR complex}

We shall now specialize the results of Sections 5.3, 5.4, and 5.5 to the case where $\mathcal{E}=T X$. This will allow us to describe the appropriate sheaf of CDOs associated with the half-twisted model on a complex, hermitian manifold $X$.

First, note that as in the twisted heterotic model, the perturbative chiral algebra $\mathcal{A}$ of local, holomorphic operators $\mathcal{F}$ in the $\bar{Q}_{+}$-cohomology of the half-twisted model can be described via Cech cohomology. In particular, let the sheaf $\widehat{\mathcal{A}}$ of chiral algebras have as its local sections the $\bar{Q}_{+}$-closed operators $\widehat{\mathcal{F}}\left(\phi^{i}, \partial_{z} \phi^{i}, \ldots ; \phi^{\bar{i}}, \partial_{z} \phi^{\bar{i}}, \ldots ; \psi_{z i}, \partial_{z} \psi_{z i}, \ldots ; \psi^{i}, \partial_{z} \psi^{i}, \ldots\right)$ that are $\psi^{\bar{i}}$-independent (i.e., $g_{\mathrm{R}}=0$ ) with arbitrary integer values of $g_{\mathrm{L}}$. Then, 
the $\bar{Q}_{+}$-cohomology of local operators can be described in terms of the Cech cohomology of $\widehat{\mathcal{A}}$ for all $g_{\mathrm{R}}$ in quantum perturbation theory; the perturbative chiral algebra $\mathcal{A}$ will thus be given by $\bigoplus_{g_{\mathrm{R}}} H_{\text {Cech }}^{g_{\mathrm{R}}}(X, \widehat{\mathcal{A}})$ as a vector space.

\subsubsection{The local action and its holomorphic structure}

Next, we shall now describe the local structure of the sheaf $\widehat{\mathcal{A}}$. Since $\mathcal{E}=$ $T X$, the local action (derived from a flat hermitian metric) of the halftwisted model on a small open set $U \subset X$ will be given by

$$
I=\frac{1}{2 \pi} \int_{\Sigma}\left|d^{2} z\right| \sum_{i, \bar{j}} \delta_{i \bar{j}}\left(\partial_{z} \phi^{\bar{j}} \partial_{\bar{z}} \phi^{i}+\psi_{z}^{\bar{j}} \partial_{\bar{z}} \psi^{i}+\psi_{\bar{z}}^{i} \partial_{z} \psi^{\bar{j}}\right)
$$

Note that on $U$, the Ricci tensor vanishes and the term containing the class $H^{1}\left(X, \Omega_{X}^{2, \mathrm{cl}}\right)$ is $\bar{Q}_{+}$-trivial. Hence, as explained in Section $5.4, \bar{Q}_{+}$acts as $\psi^{\bar{i}} \partial / \partial \phi^{\bar{i}}$, and the $\bar{Q}_{+}$-invariant operators take the form $\widehat{\mathcal{F}}\left(\phi^{i}, \partial_{z} \phi^{i}, \ldots ; \partial_{z} \phi^{\bar{i}}\right.$, $\left.\partial_{z}^{2} \phi^{i}, \ldots ; \psi_{z i}, \partial_{z} \psi_{z i}, \partial_{z}^{2} \psi_{z i}, \ldots ; \psi^{i}, \partial_{z} \psi^{i}, \partial_{z}^{2} \psi^{i}, \ldots\right)$ in the local theory with action (6.12). Also note that the operators have to be $\psi^{\bar{i}}$-independent on $U$ (see arguments in Section 5.3), in addition to being $\phi^{\bar{i}}$-independent. Clearly, the operators, in their dependence on the centre of mass coordinate of the string whose worldsheet theory is the half-twisted $(2,2)$ sigma model, are holomorphic. Therefore, the $\bar{Q}_{+}$-cohomology of operators in the chiral algebra of the local half-twisted model with action (6.12) are local sections of the sheaf of chiral algebras $\widehat{\mathcal{A}}$.

The local theory with action (6.12) has an underlying, holomorphic, twisted $N=2$ superconformal structure as follows. First, the action is invariant under the following field transformations

$$
\delta \psi^{i}=\psi^{i}, \delta \psi_{z}^{\bar{i}}=-\psi_{z}^{\bar{i}}, \quad \text { and } \quad \delta \phi^{i}=\psi^{i}, \quad \delta \psi_{z}^{\bar{i}}=-\partial_{z} \phi^{\bar{i}}
$$

where the corresponding conserved currents are given by the dimension 1 , bosonic and fermionic operators $\widehat{J}(z)$ and $\widehat{Q}(z)$, respectively. They can be written as

$$
\widehat{J}(z)=\delta_{i \bar{j}} \psi_{z}^{\bar{j}} \psi^{i} \quad \text { and } \quad \widehat{Q}(z)=\delta_{i \bar{j}} \psi^{i} \partial_{z} \phi^{\bar{j}}
$$

Note that we also have the relation $[\widehat{Q}, \widehat{J}(z)]=-\widehat{Q}(z)$, where $\widehat{Q}$ is the charge of the current $\widehat{Q}(z)$. Secondly, the conserved, holomorphic stress tensor is 
given by

$$
\widehat{T}(z)=-\delta_{i \bar{j}} \partial_{z} \phi^{i} \partial_{z} \phi^{\bar{j}}-\delta_{i \bar{j}} \psi_{z}^{\bar{j}} \partial_{z} \psi^{i},
$$

where one can derive another conserved, fermionic current $\widehat{G}(z)$, such that $\widehat{T}(z)=\{\widehat{Q}, \widehat{G}(z)\}$, and

$$
\widehat{G}(z)=\delta_{i \bar{j}} \psi_{z}^{\bar{j}} \partial_{z} \phi^{i}
$$

One can verify that $\widehat{J}(z), \widehat{Q}(z), \widehat{T}(z)$, and $\widehat{G}(z)$ satisfy the same OPE relations as that satisfied by $J(z), Q(z), T(z)$, and $G(z)$ in (6.10). In other words, they furnish the same twisted $N=2$ superconformal algebra satisfied by $J(z), Q(z), T(z)$, and $G(z)$ in the global version of the classical half-twisted sigma model with action $S_{(2,2)} . \widehat{J}(z), \widehat{Q}(z), \widehat{T}(z)$, and $\widehat{G}(z)$ are local versions of $J(z), Q(z), T(z)$, and $G(z)$, respectively. Hence, if there is no obstruction to a global definition of $\widehat{J}(z), \widehat{Q}(z), \widehat{T}(z)$, and $\widehat{G}(z)$ in the quantum theory, the symmetries associated with $J(z), Q(z), T(z)$, and $G(z)$ will persist in the non-linear half-twisted sigma model at the quantum level. Another way to see this is to first notice that $J(z), Q(z), T(z)$, and $G(z)$ are $\psi^{\bar{i}}$-independent operators and as such, will correspond to classes in $H^{0}\left(X, \widehat{\Omega}_{X}^{\text {ch }}\right)$ (from our $\bar{Q}_{+}$-Cech cohomology dictionary). Hence, these operators will exist in the $\bar{Q}_{+}$-cohomology if they correspond to global sections of $\widehat{\Omega}_{X}^{\text {ch }}$.

\subsubsection{The free $b c-\beta \gamma$ system}

Let us now set $\beta_{i}=\delta_{i \bar{j}} \partial_{z} \phi^{\bar{j}}, \gamma^{i}=\phi^{i}, \delta_{i \bar{j}} \psi_{z}^{\bar{j}}=b_{i}$, and $\psi^{i}=c^{i}$, whereby $\beta_{i}$ and $\gamma^{i}$ are bosonic operators of dimensions $(1,0)$ and $(0,0)$, while $b_{i}$ and $c^{i}$ are fermionic operators of dimension $(1,0)$ and $(0,0)$, respectively. Then, the $\bar{Q}_{+}$-cohomology of operators regular in $U$ can be represented by arbitrary local functions of $\beta, \gamma, b$, and $c$ of the form $\widehat{\mathcal{F}}\left(\gamma, \partial_{z} \gamma, \partial_{z}^{2} \gamma, \ldots, \beta, \partial_{z} \beta, \partial_{z}^{2} \beta, \ldots\right.$, $\left.b, \partial_{z} b, \partial_{z}^{2} b, \ldots, c, \partial_{z} c, \partial_{z}^{2} c, \ldots\right)$. The operators $\beta$ and $\gamma$ have the operator products of a standard $\beta \gamma$ system. The products $\beta \cdot \beta$ and $\gamma \cdot \gamma$ are nonsingular, while

$$
\beta_{i}(z) \gamma^{j}\left(z^{\prime}\right)=-\frac{\delta_{i j}}{z-z^{\prime}}+\text { regular. }
$$

Similarly, the operators $b$ and $c$ have the operator products of a standard $b c$ system. The products $b \cdot b$ and $c \cdot c$ are non-singular, while

$$
b_{i}(z) c^{j}\left(z^{\prime}\right)=\frac{\delta_{i j}}{z-z^{\prime}}+\text { regular. }
$$

These statements can be deduced from the flat action (6.12) by standard methods. We can write down an action for the fields $\beta, \gamma, b$, and $c$, regarded 
as free elementary fields, which reproduces these OPEs. It is simply the standard action of a $b c-\beta \gamma$ system:

$$
I_{b c-\beta \gamma}=\frac{1}{2 \pi} \int\left|d^{2} z\right| \sum_{i}\left(\beta_{i} \partial_{\bar{z}} \gamma^{i}+b_{i} \partial_{\bar{z}} c^{i}\right) .
$$

Hence, we find that the linear (i.e., local) version of the $b c-\beta \gamma$ system above reproduces the $\bar{Q}_{+}$-cohomology of $\psi^{\bar{i}}$-independent operators of the half-twisted model on $U$, i.e., the local sections of $\widehat{\mathcal{A}}$.

At this point, one can make some important observations about the relationship between the symmetries of the local half-twisted $(2,2)$ model with action (6.12) and the symmetries of the local version of the $b c-\beta \gamma$ system above. Note that the free $b c-\beta \gamma$ action (6.19) is invariant under the following field variations

$$
\delta c^{i}=c^{i}, \delta b_{i}=-b_{i} \quad \text { and } \quad \delta \gamma^{i}=c^{i}, \delta b_{i}=-\beta_{i},
$$

where the corresponding conserved, bosonic, and fermionic currents will be given by $\mathcal{J}(z)$ and $\mathcal{Q}(z)$, respectively. They can be written as

$$
\mathcal{J}(z)=b_{i} c^{i} \text { and } \mathcal{Q}(z)=\beta_{i} c^{i}
$$

In addition, we have the relation $[\mathcal{Q}, \mathcal{J}(z)]=-\mathcal{Q}(z)$, where $\mathcal{Q}$ is the charge of the current $\mathcal{Q}(z)$. The action is also invariant under

$$
\delta c^{i}=\partial_{z} \gamma^{i} \quad \text { and } \quad \delta \beta_{i}=\partial_{z} b_{i}
$$

where the corresponding conserved, fermionic current will be given by

$$
\mathcal{G}(z)=b_{i} \partial_{z} \gamma^{i} \text {. }
$$

Finally, the stress tensor of the local $b c-\beta \gamma$ system is

$$
\mathcal{T}(z)=-\beta_{i} \partial_{z} \gamma^{i}-b_{i} \partial_{z} c^{i}
$$

where we also have the relation $\{\mathcal{Q}, \mathcal{G}(z)\}=\mathcal{T}(z)$. (Note that we have omitted the normal-ordering symbol in writing the above conserved currents and tensor.) One can verify that just like the fields $\widehat{J}(z), \widehat{Q}(z), \widehat{G}(z)$, and $\widehat{T}(z)$, the fields $\mathcal{J}(z), \mathcal{Q}(z), \mathcal{G}(z)$ and $\mathcal{T}(z)$ generate a holomorphic, twisted $N=2$ superconformal algebra. In fact, via the respective identification of the fields $\beta$ and $\gamma$ with $\partial_{z} \phi$ and $\phi, \psi_{z i}$ and $\psi^{i}$ with $b_{i}$ and $c^{i}$, we find that $\widehat{J}(z), \widehat{Q}(z)$, $\widehat{T}(z)$, and $\widehat{G}(z)$ coincide with $\mathcal{J}(z), \mathcal{Q}(z), \mathcal{T}(z)$, and $\mathcal{G}(z)$, respectively. This observation will be important in Section 7.1, when we consider an explicit example.

The $b c-\beta \gamma$ system above will certainly reproduce the $\bar{Q}_{+}$-cohomology of $\psi^{\bar{i}}$-independent operators globally on $X$ if its non-linear version is anomalyfree. In the non-linear $b c-\beta \gamma$ system with action (6.19), one must interpret $\gamma$ as a map $\gamma: \Sigma \rightarrow X, \beta$ as a $(1,0)$-form on $\Sigma$ with values in the pull-back 
$\gamma^{*}\left(T^{*} X\right)$, the fermionic field $c$ as a scalar on $\Sigma$ with values in the pull-back $\gamma^{*}(T X)$, and the fermionic field $b$ as a $(1,0)$-form on $\Sigma$ with values in the pull-back $\gamma^{*}\left(T^{*} X\right)$. Following the same arguments in Section 5.4, we find that the kinetic operators associated with the fields of the non-linear $b c-\beta \gamma$ system are effectively given by $D$ and $\bar{D}$. Moreover, they act on sections of the same pull-back bundle $\gamma_{0}^{*}(T X)$. Hence, via the analysis of Section 4, we find that the anomalies of the non-linear $b c-\beta \gamma$ system vanish. This is consistent with the absence of anomalies in the underlying half-twisted model. Thus, one can find global sections of $\widehat{\mathcal{A}}$.

Locally on $X$, the $\bar{Q}_{+}$-cohomology of the half-twisted model is nonvanishing only for $g_{\mathrm{R}}=0$. However, there can generically be cohomology in higher degrees globally on $X$. Nevertheless, as explained in Section 5.4, the $\bar{Q}_{+}$-cohomology classes of positive degree (i.e., $g_{\mathrm{R}}>0$ ) can still be described in the framework of the free $b c-\beta \gamma$ system via Cech cohomology - the operators with degree $g_{\mathrm{R}}>0$ can be represented as Cech- $g_{\mathrm{R}}$ cocycles that generate the $g_{\mathrm{R}}{ }^{\text {th }}$ Cech cohomology of the sheaf $\widehat{\mathcal{A}}$ of the chiral algebra of the linear $b c-\beta \gamma$ system (with action a linearized version of (6.19)).

As for the moduli of the theory, the complex structure is built into the definition of the fields in (6.19). The moduli of the chiral algebra $\mathcal{A}$, given by a class in $H^{1}\left(X, \Omega_{X}^{2, c l}\right)$, are built into the definition of Cech 1-cocycles that represent the admissible automorphisms of the sheaves of free conformal fields theories (see Section 5.6).

By specializing the arguments in Section 5.4 to $\mathcal{E}=T X$, we shall now discuss the computation of a correlation function of cohomology classes of local operators within the framework of the free $b c-\beta \gamma$ system. As explained in Section 5.4, due to a right-moving ghost number anomaly, for generic correlation functions in perturbation theory to be non-vanishing, it is a requirement that some of the local operators have positive degrees. Hence, from our description above, the computation of the correlation functions will involve cup products of Cech cohomology groups and their corresponding maps into complex numbers. We can illustrate this scheme by computing a generic correlation function of dimension $(0,0)$ operators on a genus-zero Riemann surface such as a sphere. To this end, recall from Section 6.1 that a dimension $(0,0)$ operator $\mathcal{O}_{i}$ with ghost number $\left(g_{\mathrm{L}}, g_{\mathrm{R}}\right)=\left(p_{i}, q_{i}\right)$ can be interpreted as a $\left(0, q_{i}\right)$-form with values in the holomorphic bundle $\Lambda^{p_{i}} T^{*} X$. Thus, it represents a class in the Cech cohomology group $H^{q_{i}}\left(X, \Lambda^{p_{i}} T^{*} X\right)$. Note that due to the additional left-moving ghost number anomaly, the correlation functions of our model must also satisfy $\sum_{i} p_{i}=\sum_{i} q_{i}=\operatorname{dim}_{\mathbb{C}} X=n$ in perturbation theory, so as to be non-vanishing on the sphere. Since the half-twisted model is restricted to holomorphic maps with regard to the fixed-point theorem, we find that the correlation function path integral 
reduces to an integral over the moduli space of holomorphic maps. Because we are considering degree-zero maps in perturbation theory, the moduli space of holomorphic maps will be $X$ itself, i.e., the path integral reduces to an integral over the target space $X$. In summary, we find that a nonvanishing perturbative correlation function involving $s$ dimension $(0,0)$ operators $\mathcal{O}_{1}, \mathcal{O}_{2}, \ldots, \mathcal{O}_{s}$ on the sphere, can be computed as

$$
\left\langle\mathcal{O}_{1}\left(z_{1}\right) \cdots \mathcal{O}_{s}\left(z_{s}\right)\right\rangle_{0}=\int_{X} \mathcal{W}^{n, n}
$$

where $\mathcal{W}^{n, n}$ is a top-degree form on $X$ which represents a class in the Cech cohomology group $H^{n}\left(X, \Lambda^{n} T^{*} X\right)$. This $(n, n)$-form is obtained via the sequence of maps

$$
\begin{aligned}
& H^{q_{1}}\left(X, \Lambda^{p_{1}} T^{*} X\right) \otimes \cdots \otimes H^{q_{s}}\left(X, \Lambda^{p_{s}} T^{*} X\right) \rightarrow H^{n}\left(X, \otimes_{i=1}^{s} \Lambda^{p_{i}} T^{*} X\right) \\
& \quad \rightarrow H^{n}\left(X, \Lambda^{n} T^{*} X\right),
\end{aligned}
$$

where $\sum_{i=1}^{s} q_{i}=\sum_{i=1}^{s} p_{i}=n$. The first map is given by the cup product of Cech cohomology classes which represent the corresponding dimension $(0,0)$ operators, while the second map is given by a wedge product of exterior powers of the holomorphic cotangent bundle. Similar procedures will apply in the computation of correlation functions of local operators with higher dimension.

Note that in order to compute a non-perturbative correlation function of dimension $(0,0)$ operators with $\left(g_{\mathrm{L}}, g_{\mathrm{R}}\right)=\left(p_{i}, q_{i}\right)$, the operators must instead be represented by Cech cohomology classes $H^{q_{i}}\left(\mathcal{M}, \Lambda^{p_{i}} T^{*} \mathcal{M}\right)$ in the moduli space $\mathcal{M}$ of worldsheet instantons. ${ }^{25}$ An extension of this recipe to compute the non-perturbative correlation functions of local operators of higher dimension, will therefore serve as the basis of a chiral version of quantum cohomology.

\subsubsection{The sheaf $\widehat{\Omega}_{X}^{\text {ch }}$ of CDR complex on $X$}

In the case of the twisted heterotic sigma model, where $\mathcal{E} \neq T X$ but is equivalent to some arbitrary holomorphic vector bundle over $X$, we showed in Section 5.5 that the relevant, free $b c-\beta \gamma$ system with action (5.14) will reproduce the vertex superalgebras spanned by CDOs on the exterior algebra $\Lambda \mathcal{E}$. One may then ask the following question: in the case of the halftwisted model, what kind of vertex superalgebra does the free $b c-\beta \gamma$ system with action (6.19) reproduce? Or alternatively, what kind of sheaf does $\widehat{\mathcal{A}}$ mathematically describe in this instance?

\footnotetext{
${ }^{25}$ This means that the Cech cohomology classes in $X$ of (6.26) will be replaced by Cech cohomology classes in the moduli space of worldsheet instantons (See [15]).
} 
In order to ascertain this, one must first and foremost determine the admissible automorphisms of the free $b c-\beta \gamma$ system with action (6.19) (as was done for the $\mathcal{E} \neq T X$ case in Section 5.5). Since we are considering $\mathcal{E}=T X$, the components of the transition function matrix of the holomorphic bundle, given by $A_{j}{ }^{i}$ in Section 5.5, will now generate holomorphic coordinate transformations on $X$. In other words, we must make the following replacements in $(5.22)-(5.25)$ :

$$
\begin{aligned}
A_{n}{ }^{l} & =\frac{\partial g^{l}}{\partial \gamma^{n}}, \\
\left(\partial_{k} A^{-1}\right)_{l}{ }^{m} & =\frac{\partial^{2} \gamma^{m}}{\partial \gamma^{k} \partial g^{l}}, \\
D^{k}{ }_{i} & =\frac{\partial \gamma^{k}}{\partial g^{i}},
\end{aligned}
$$

where $g^{i}(\gamma)=\widetilde{\gamma}^{i}$, and $i, j, k, l, m, n=1,2, \ldots, \operatorname{dim}_{\mathbb{C}} X$. As the obstruction to gluing of sheaves vanishes for any $X$ when describing the half-twisted $(2,2)$ model, it will mean that from our discussion in Section 5.5 on the local symmetries of the associated free $b c-\beta \gamma$ system, the extension of groups given by (5.29) will be trivial. Thus, the universal cohomology group $H^{2}\left(\widetilde{H}, \Omega_{\widetilde{H}}^{2, \text { cl }}\right)$, which characterizes the extension's non-triviality, will also vanish. As expla ined in Section 5.5, this will mean that $\widetilde{G}=\widetilde{H}$, i.e., the admissible automorphisms of the associated free $b c-\beta \gamma$ system are solely generated by $\widetilde{H}$. Consequently, the last term on the RHS of (5.23) can be set to zero in the present computation. Hence, the admissible automorphisms of the free $b c$ $\beta \gamma$ system, which describes the half-twisted $(2,2)$ model locally on $X$, will be given by

$$
\begin{aligned}
& \tilde{\gamma}^{i}=g^{i}(\gamma), \\
& \tilde{\beta}_{i}=\frac{\partial \gamma^{k}}{\partial \widetilde{\gamma}^{i}} \beta_{k}+\frac{\partial^{2} \gamma^{l}}{\partial \widetilde{\gamma}^{i} \partial \widetilde{\gamma}^{j}} \frac{\partial \widetilde{\gamma}^{j}}{\partial \gamma^{k}} b_{l} c^{k}, \\
& \tilde{c}^{i}=\frac{\partial \widetilde{\gamma}^{i}}{\partial \gamma^{k}} c^{k}, \\
& \tilde{b}_{i}=\frac{\partial \gamma^{k}}{\partial \widetilde{\gamma}^{i}} b_{k},
\end{aligned}
$$

where $i, j, k, l=1,2, \ldots, \operatorname{dim}_{\mathbb{C}} X$. The field transformations (6.30)-(6.33) coincide with (3.17a)-(3.17d) of [1], which define the admissible automorphisms of a sheaf of conformal vertex superalgebras mathematically known as the CDR complex. Hence, we learn that $\widehat{\mathcal{A}}$ is the sheaf of CDR complex on $X$. The identification of $\widehat{\mathcal{A}}$ with a sheaf conformal vertex superalgebras is indeed consistent with the fact that the conformal anomaly of Section 5.7 vanishes for the half-twisted model. We shall henceforth label the sheaf of 
chiral algebras $\widehat{\mathcal{A}}$, associated with the half-twisted $(2,2)$ model on $X$, as the sheaf $\widehat{\Omega}_{X}^{\text {ch }}$ of CDR complex on $X$, or CDR for short. Thus, the chiral algebra $\mathcal{A}$ of the half-twisted $(2,2)$ model is, as a vector space, given by $\bigoplus_{g_{\mathrm{R}}} H_{\mathrm{Cech}}^{g_{\mathrm{R}}}\left(X, \widehat{\Omega}_{X}^{\text {ch }}\right)$. It is satisfying to note that the above results and conclusion serve as an alternative verification and generalization of Kapustin's findings in [11] for a Calabi-Yau manifold.

\subsection{The elliptic genus of the half-twisted $(2,2)$ model}

In this section, we will discuss the elliptic genus of the half-twisted $(2,2)$ sigma model on $X$ from a purely physical perspective. In the process, we will be able to relate it to a corresponding definition in the mathematical literature, thereby supporting the consistency of our interpretation of the $\bar{Q}_{+}$-cohomology classes in the chiral algebra $\mathcal{A}$ as classes of the Cech cohomology generated by the sheaf $\widehat{\Omega}_{X}^{\text {ch }}$ of CDR complex on $X$.

Physically, the elliptic genus is a certain specialization of the partition function of the half-twisted $(2,2)$ model, with worldsheet being a torus with modulus $\tau$. It counts the number of supersymmetric (BPS) states with $\bar{L}_{0}=0$ or rather the right-moving ground states. These are simply the states in the $\bar{Q}_{+}$-cohomology of the half-twisted model. (Recall that we discussed this in Section 3.1.) The elliptic genus is also known to coincide with the Euler characteristic of $X$. Consequently, it is a topological invariant of $X$, and it can be written as a function of two variables $y$ and $q$ as [29]

$$
\chi(X, y, q)=\operatorname{Tr}_{\mathcal{H}}(-1)^{F} y^{J_{\mathrm{L}}} q^{L_{0}-d / 8}
$$

where $d=\operatorname{dim}_{\mathbb{C}} X, q=\mathrm{e}^{2 \pi i \tau}$, and $y=\mathrm{e}^{2 \pi i z}$, with $z$ being a point in a Jacobian that determines the line-bundle of which the fermions of the theory are sections thereof. $F=F_{\mathrm{L}}+F_{\mathrm{R}}$ is the total fermion number, $\mathcal{H}$ is the Hilbert space obtained via quantizing the loop space $\mathcal{L} X$, while $\operatorname{Tr}_{\mathcal{H}}(-1)^{F}$ is the Witten index that counts the difference between the number of bosonic and fermionic states at each energy level $n$. The $U(1)$ charge $J_{\mathrm{L}}$ is actually the left-moving ghost number $g_{\mathrm{L}}$. (We have renamed it here to allow (6.34) to take its standard form as found in the physics literature.)

Notice that the above discussion on the elliptic genus involves the states but not the operators in the half-twisted model. When and how do the local operators come into the picture? In order to associate the elliptic genus with the local operators in the chiral algebra of the sigma model, one has to consider the canonical quantization of the sigma model on an infinitely long cylinder $\mathbb{R} \times S^{1}$. If $c_{1}(X)=0$, one can proceed to employ the CFT stateoperator isomorphism, from which one can then obtain a correspondence 
between the above states and operators. The elliptic genus can thus be expressed in terms of the difference between the number, of bosonic and fermionic operators in the $\bar{Q}_{+}$-cohomology, such that the holomorphic (i.e., left-moving) dimension of the operators $n$ will now correspond to the energy level $n$ of the supersymmetric states that the operators are isomorphic to. Note that if $c_{1}(X) \neq 0$, the state-operator correspondence will not be an isomorphism. Rather, the states just furnish a module $\mathcal{V}$ of the chiral algebra $\mathcal{A}$ of local operators, whereby $\mathcal{V}$ is only isomorphic to $\mathcal{A}$ if $c_{1}(X)=0$. Based on the above correspondence, the description of $\mathcal{A}$ in terms of the Cech cohomology of $\widehat{\Omega}_{X}^{\text {ch }}$, and the fact that bosonic and fermionic operators have even and odd total ghost numbers $g_{\mathrm{L}}+g_{\mathrm{R}}$, respectively, we find that in the smooth Calabi-Yau case (i.e., $c_{1}(X)=0$ ), the elliptic genus in (6.34) can be written as

$$
\chi_{c}(X, y, q)=q^{-d / 8} \sum_{g_{\mathrm{L}}, g_{\mathrm{R}}} \sum_{n=0}^{\infty}(-1)^{g_{\mathrm{L}}+g_{\mathrm{R}}} \operatorname{dim} H^{g_{\mathrm{R}}}\left(X, \widehat{\Omega}_{X ; n}^{\mathrm{ch} ; g_{\mathrm{L}}}\right) y^{g_{\mathrm{L}}} q^{n},
$$

where $\widehat{\Omega}_{X, n}^{\mathrm{ch}, g_{\mathrm{L}}}$ is a sheaf of CDR on $X$, whose local sections correspond to the $\psi^{\bar{i}}$-independent $\bar{Q}_{+}$-cohomology classes with dimension $(n, 0)$ and leftmoving ghost number $g_{\mathrm{L}}$.

Mathematically, the elliptic genus can be understood as the $S^{1}$-equivariant Hirzebruch $\chi_{y}$-genus of the loop space of $X$. If $X$ is Calabi-Yau, the elliptic genus will have nice modular properties under $S L(2, \mathbb{Z})$. Now, let us define a sheaf of CDR on a smooth Calabi-Yau $X$. Then, the mathematical definition of the elliptic genus, written as $\operatorname{Ell}(X, y, q)$, will take the form $[6]$

$$
\operatorname{Ell}(X, y, q)=y^{-d / 2} \sum_{a, b, i}(-1)^{a+b} \operatorname{dim} H^{a}\left(X, \widehat{\Omega}_{X ; n}^{\mathrm{ch} ; b}\right) y^{b} q^{i} .
$$

Notice that we have the relation $\chi_{c}(X, y, q)=y^{d / 2} q^{-d / 8} \operatorname{Ell}(X, y, q)$. Hence, on Calabi-Yau manifolds, we find that the physical elliptic genus $\chi_{c}(X, y, q)$ can be expressed in terms of the mathematically defined elliptic genus $\operatorname{Ell}(X, y, q)$. In fact, although not readily noticeable via our current discussion, $\chi_{c}(X, y, q)$ and $\operatorname{Ell}(X, y, q)$ actually coincide. This is because the derivation of $\operatorname{Ell}(X, y, q)$ in [6] is based upon an alternative but equivalent definition of $\chi_{c}(X, y, q)$ given by [30]

$$
\chi_{c}(X, y, q)=\int \operatorname{ch}(\operatorname{Ell}) \operatorname{td}(X),
$$

with the formal sum of vector bundles

$$
\begin{gathered}
\text { Ell }=y^{-d / 2} \otimes_{k \geq 1} \wedge\left(y q^{k-1} T^{*} X\right) \otimes_{k \geq 1} \wedge\left(y^{-1} q^{k} T X\right) \\
\otimes_{k \geq 1} \operatorname{Sym}\left(q^{k} T^{*} X\right) \otimes_{k \geq 1} \operatorname{Sym}\left(q^{k} T X\right),
\end{gathered}
$$


where $\operatorname{td}(X)$ refers to the Todd class of the holomorphic tangent bundle $T X$, and the symbol "Sym" just denotes the symmetrized product of bundles.

Notice that $\chi_{c}(X, q, y)$ is $\mathbb{Z}_{\geq 0} \times \mathbb{Z}$ graded by the holomorphic dimension $n$ and left-moving ghost number $g_{\mathrm{L}}$ of the $\bar{Q}_{+}$-invariant operators, respectively. The grading by dimension follows naturally from the scale invariance of the correlation functions and the chiral algebra $\mathcal{A}$ of the half-twisted model. Note that $\chi_{c}(X, y, q)$ has no perturbative quantum corrections. ${ }^{26}$ However, if $c_{1}(X) \neq 0$, non-perturbative worldsheet instanton corrections may violate the scale invariance of the correlation functions and hence, the grading by dimension of the operators in $\mathcal{A} .^{27}$ Consequently, supersymmetry may be spontaneously broken, thus rendering $\mathcal{V}$ empty, as all the bosonic and fermionic operators pair up.

\section{Examples of sheaves of CDR}

In this section, we study in detail, examples of sheaves of CDR and their cohomologies on two different smooth manifolds. Our main objective is to illustrate the rather abstract discussion in Section 6. In the process, we will again obtain an interesting and novel understanding of the relevant physics in terms of pure mathematical data.

\subsection{The sheaf of CDR on $\mathbb{C P}^{1}$}

For our first example, following part III of [2], we take $X=\mathbb{C P}^{1}$. In other words, we will be exploring and analysing the chiral algebra $\mathcal{A}$ of operators in the half-twisted $(2,2)$ model on $\mathbb{C P}^{1}$. To this end, we will work locally on the worldsheet $\Sigma$, choosing a local complex parameter $z$.

As mentioned, $\mathbb{C P}^{1}$ can be regarded as the complex $\gamma$-plane plus a point at infinity. Thus, we can cover it by two open sets, $U_{1}$ and $U_{2}$, where $U_{1}$ is the complex $\gamma$-plane and $U_{2}$ is the complex $\tilde{\gamma}$-plane, where $\tilde{\gamma}=1 / \gamma$.

\footnotetext{
${ }^{26}$ Absence of quantum corrections can be inferred from the fact that both the energy and the $(-1)^{F}$ operator that distinguishes the bosonic and fermionic states are exactly conserved quantum mechanically.

${ }^{27}$ In the non-perturbative small radius limit, if $c_{1}(X) \neq 0$, the contribution from worldsheet instantons (resulting from a pull-back of the $(1,1)$-form $\omega_{T}$ on holomorphic curves) will serve to renormalize $\omega_{T}$. This gives rise to dimensional transmutation, whereby the exponential of $\omega_{T}$, which appears in the non-perturbative correlation functions, will be replaced by a dimensionful scale parameter $\Lambda$.
} 
Since $U_{1}$ is isomorphic to $\mathbb{C}$, the sheaf of CDR in $U_{1}$ can be described by a single free $b c-\beta \gamma$ system with action

$$
I=\frac{1}{2 \pi} \int\left|d^{2} z\right| \beta \partial_{\bar{z}} \gamma+b \partial_{\bar{z}} c .
$$

Here $\beta, b$, and $c, \gamma$, are fields of dimension $(1,0)$ and $(0,0)$, respectively. They obey the usual free-field OPEs; there are no singularities in the operator products $\beta(z) \cdot \beta\left(z^{\prime}\right), b(z) \cdot b\left(z^{\prime}\right), \gamma(z) \cdot \gamma\left(z^{\prime}\right)$, and $c(z) \cdot c\left(z^{\prime}\right)$, while

$$
\beta(z) \gamma\left(z^{\prime}\right) \sim-\frac{1}{z-z^{\prime}} \quad \text { and } \quad b(z) c\left(z^{\prime}\right) \sim \frac{1}{z-z^{\prime}} .
$$

Similarly, the sheaf of CDR in $U_{2}$ is described by a single free $\tilde{b} \tilde{c}-\tilde{\beta} \tilde{\gamma}$ system with action

$$
I=\frac{1}{2 \pi} \int\left|d^{2} z\right| \tilde{\beta} \partial_{\bar{z}} \tilde{\gamma}+\tilde{b} \partial_{\bar{z}} \tilde{c}
$$

where the fields $\tilde{\beta}, \tilde{b}, \tilde{\gamma}$, and $\tilde{c}$ obey the same OPEs as $\beta, b, \gamma$, and $c$. In other words, the non-trivial OPEs are given by

$$
\tilde{\beta}(z) \tilde{\gamma}\left(z^{\prime}\right) \sim-\frac{1}{z-z^{\prime}} \quad \text { and } \quad \tilde{b}(z) \tilde{c}\left(z^{\prime}\right) \sim \frac{1}{z-z^{\prime}} .
$$

In order to describe a globally defined sheaf of CDR, one will need to glue the free conformal field theories with actions (7.1) and (7.3) in the overlap region $U_{1} \cap U_{2}$. To do so, one must use the admissible automorphisms of the free conformal field theories defined in (6.30)-(6.33) to glue the free-fields together. In the case of $X=\mathbb{C P}^{1}$, the automorphisms will be given by

$$
\begin{aligned}
\tilde{\gamma} & =\frac{1}{\gamma}, \\
\tilde{\beta} & =-\gamma^{2} \beta-2 \gamma b c, \\
\tilde{c} & =-\frac{c}{\gamma^{2}}, \\
\tilde{b} & =-\gamma^{2} b .
\end{aligned}
$$

As there is no obstruction to this gluing in the half-twisted model, a sheaf of CDR can be globally defined on the target space $\mathbb{C P}^{1}$ (but only locally defined on the worldsheet $\Sigma$ of the conformal field theory, because we are using a local complex parameter $z$ to define it).

\subsubsection{Global sections of the sheaf}

Recall that for a general manifold $X$ of complex dimension $n$, the chiral algebra $\mathcal{A}$ will be given by $\mathcal{A}=\bigoplus_{g_{\mathrm{R}}=0}^{g_{\mathrm{R}}=n} H^{g_{\mathrm{R}}}\left(X, \widehat{\Omega}_{X}^{\text {ch }}\right)$ as a vector space. Since $\mathbb{C P}^{1}$ has complex dimension 1 , we will have, for $X=\mathbb{C P}^{1}$, the relation $\mathcal{A}=$ 
$\bigoplus_{g_{\mathrm{R}}=0}^{g_{\mathrm{R}}=1} H^{g_{\mathrm{R}}}\left(\mathbb{C P}^{1}, \widehat{\Omega}_{\mathbb{P}^{1}}^{\mathrm{ch}}\right)$. Thus, in order to understand the chiral algebra of the half-twisted model, one needs only to study the global sections of the sheaf $\widehat{\Omega}_{\mathbb{P}^{1}}^{\text {ch }}$ and its first Cech cohomology $H^{1}\left(\mathbb{C P}^{1}, \widehat{\Omega}_{\mathbb{P}^{1}}^{\text {ch }}\right)$.

First, let us consider $H^{0}\left(\mathbb{C P}^{1}, \widehat{\Omega}_{\mathbb{P}^{1}}^{\text {ch }}\right)$, the global sections of $\widehat{\Omega}_{\mathbb{P}^{1}}^{c h}$. At dimension 0 , the space of global sections $H^{0}\left(\mathbb{C P}^{1}, \widehat{\Omega}_{\mathbb{P}^{1} ; 0}^{\text {ch;* }}\right)$ must be spanned by functions of $\gamma$ and/or $c$ only. Note that it can be a function of higher degree in $\gamma$, but only a function of single degree in $c$ - higher powers of $c$ vanish (since $c^{2}=0$ ) because it is fermionic. In other words, the global sections are given by $H^{0}\left(\mathbb{C P}^{1}, \widehat{\Omega}_{\mathbb{P}^{1} ; 0}^{\text {ch } g_{\mathrm{L}}}\right)$, where $g_{\mathrm{L}}=0$ or 1 . Notice that $\widehat{\Omega}_{\mathbb{P}^{1} ; 0}^{\text {ch; }}$ is just the sheaf $\mathcal{O}$ of holomorphic functions in $\gamma$ on $\mathbb{C P}^{1}$ and that classically (from ordinary algebraic geometry), we have the result $H^{1}\left(\mathbb{C P}^{1}, \mathcal{O}\right)=0$. Since a vanishing cohomology in the classical theory continues to vanish in quantum theory, $H^{1}\left(\mathbb{C P}^{1}, \widehat{\Omega}_{\mathbb{P}^{1} ; 0}^{\text {ch;0 }}\right)=0$ will hold in quantum perturbation theory. From the chiral Poincaré duality [31], we have the relation $H^{0}\left(\mathbb{C P}^{1}, \widehat{\Omega}_{\mathbb{P}^{1} ; 0}^{\mathrm{ch} ; 1}\right)^{*}=$ $H^{1}\left(\mathbb{C P}^{1}, \widehat{\Omega}_{\mathbb{P}^{1} ; 0}^{\text {ch } 0}\right)$. This means that $H^{0}\left(\mathbb{C P}^{1}, \widehat{\Omega}_{\mathbb{P}^{1} ; 0}^{\mathrm{ch} ; 1}\right)=0$, or rather, the global sections at dimension 0 are holomorphic functions in $\gamma$ only. Since all regular, holomorphic functions on a compact Riemann surface such as $\mathbb{C P}^{1}$ must be constants, we find that the space of global sections at dimension 0 , given by $H^{0}\left(\mathbb{C P}^{1}, \widehat{\Omega}_{\mathbb{P}^{1} ; 0}^{\mathrm{ch} ;}\right)$, is one-dimensional and generated by 1 .

Let us now ascertain the space $H^{0}\left(\mathbb{C P}^{1}, \widehat{\Omega}_{\mathbb{P}_{1}^{1} ; 1}^{\mathrm{ch} ;}\right)$ of global sections of dimension 1 . In order to get a global section of $\widehat{\Omega}_{\mathbb{P}^{1}}^{\text {ch }}$ of dimension 1 , we can act on a global section of $\widehat{\Omega}_{\mathbb{P} 1}^{\text {ch }}$ of dimension 0 with the partial derivative $\partial_{z}$. Since $\partial_{z} 1=0$, this prescription will not apply here.

One could also consider operators of the form $f(\gamma) \partial_{z} \gamma$, where $f(\gamma)$ is a holomorphic function of $\gamma$. However, there are no such global sections either - such an operator, by virtue of the way it transforms purely geometrically under (7.5), would correspond to a section of $\Omega^{1}\left(\mathbb{C P}^{1}\right)$, the sheaf of holomorphic differential forms $f(\gamma) d \gamma$ on $\mathbb{C P}^{1}$, and from the classical result $H^{0}\left(\mathbb{C P}^{1}, \Omega^{1}\left(\mathbb{C P}^{1}\right)\right)=0$, which continues to hold in the quantum theory, we see that $f(\gamma) \partial_{z} \gamma$ cannot be a dimension 1 global section of $\widehat{\Omega}_{\mathbb{P}^{1}}^{\mathrm{ch}}$.

Other possibilities include operators which are linear in $b, \partial_{z} c$, or $\beta$. In fact, from the automorphism relation of (7.6), we find an immediate example as the LHS, $\tilde{\beta}$, is by definition regular in $U_{2}$, while the RHS, being polynomial in $\gamma, b$, and $c$, is manifestly regular in $U_{1}$. Their being equal means that they represent a dimension 1 global section of $\widehat{\Omega}_{\mathbb{P} 1}^{\text {ch }}$ that we will call $J_{-}$:

$$
J_{-}=-\gamma^{2} \beta-2 \gamma b c=\tilde{\beta} .
$$


The construction is completely symmetric between $U_{1}$ and $U_{2}$, with $\gamma \leftrightarrow \tilde{\gamma}$, $\beta \leftrightarrow \tilde{\beta}, b \leftrightarrow \tilde{b}$, and $c \leftrightarrow \tilde{c}$, so a reciprocal formula gives another dimension 1 global section $J_{+}$:

$$
J_{+}=\beta=-\tilde{\gamma}^{2} \tilde{\beta}-2 \tilde{\gamma} \tilde{b} \tilde{c} .
$$

Hence, $J_{+}$and $J_{-}$give us two dimension 1 global sections of the sheaf $\widehat{\Omega}_{\mathbb{P}^{1}}^{\text {ch }}$. Since these are global sections of a sheaf of chiral vertex operators, we can construct more of them from their OPEs. There are no singularities in the $J_{+} \cdot J_{+}$or $J_{-} \cdot J_{-}$operator products, but

$$
J_{+} J_{-} \sim \frac{2 J_{3}}{z-z^{\prime}}
$$

where $J_{3}$ is another global section of dimension 1 given by

$$
J_{3}=\gamma \beta+b c .
$$

(Note that normal ordering is again understood for all operators above and below).

Similarly, from (7.8), $\tilde{b}$ is by definition regular in $U_{2}$, while the RHS, being polynomial in $\gamma$ and $b$, is manifestly regular in $U_{1}$. Their being equal means that they represent a dimension 1 global section of $\widehat{\Omega}_{\mathbb{P}^{1}}^{\text {ch }}$ that we will call $j_{-}$:

$$
j_{-}=-\gamma^{2} b=\tilde{b}
$$

Again, the construction is completely symmetric between $U_{1}$ and $U_{2}$, with $\gamma \leftrightarrow \tilde{\gamma}$ and $b \leftrightarrow \tilde{b}$, so a reciprocal formula gives another dimension 1 global section $j_{+}$:

$$
j_{+}=b=-\tilde{\gamma}^{2} \tilde{b}
$$

Similarly, since these are also global sections of a sheaf of chiral vertex operators, we can construct more of them from their OPEs. There are no singularities in the $j_{a} \cdot j_{b}$ operator products for $a, b=+$ or - . However, we do have

$$
J_{+} j_{-} \sim \frac{2 j_{3}}{z-z^{\prime}},
$$

where $j_{3}$ is another global section of dimension 1 given by

$$
j_{3}=\gamma b
$$

Notice that $\left\{J_{+}, J_{-}, J_{3}\right\}$ are bosonic operators that belong in $H^{0}\left(\mathbb{C P}^{1}, \widehat{\Omega}_{\mathbb{P}^{1} ; 1}^{\text {ch } 0}\right)$, while $\left\{j_{+}, j_{-}, j_{3}\right\}$ are fermionic operators that belong in $H^{0}\left(\mathbb{C P}^{1}, \widehat{\Omega}_{\mathbb{P}^{1} ; 1}^{\mathrm{ch} ;-1}\right)$. One can compute that they satisfy the following closed 
OPE algebra:

$$
\begin{aligned}
J_{a}(z) J_{a}\left(z^{\prime}\right) & \sim \text { regular, } \quad J_{3}(z) J_{+}\left(z^{\prime}\right) \sim \frac{+J_{+}\left(z^{\prime}\right)}{z-z^{\prime}}, \\
J_{3}(z) J_{-}\left(z^{\prime}\right) & \sim \frac{-J_{-}\left(z^{\prime}\right)}{z-z^{\prime}}, \quad J_{+}(z) J_{-}\left(z^{\prime}\right) \sim \frac{2 J_{3}\left(z^{\prime}\right)}{z-z^{\prime}}, \\
J_{3}(z) j_{-}\left(z^{\prime}\right) & \sim \frac{-j_{-}\left(z^{\prime}\right)}{z-z^{\prime}}, \quad J_{3}(z) j_{+}\left(z^{\prime}\right) \sim \frac{+j_{+}\left(z^{\prime}\right)}{z-z^{\prime}}, \\
J_{+}(z) j_{-}\left(z^{\prime}\right) & \sim \frac{2 j_{3}\left(z^{\prime}\right)}{z-z^{\prime}}, \quad J_{+}(z) j_{3}\left(z^{\prime}\right) \sim \frac{-j_{+}\left(z^{\prime}\right)}{z-z^{\prime}}, \\
J_{-}(z) j_{+}\left(z^{\prime}\right) & \sim \frac{-2 j_{3}\left(z^{\prime}\right)}{z-z^{\prime}}, \quad J_{-}(z) j_{3}\left(z^{\prime}\right) \sim \frac{j_{-}\left(z^{\prime}\right)}{z-z^{\prime}}, \\
j_{a}(z) j_{b}\left(z^{\prime}\right) & \sim \operatorname{regular}, \quad J_{a}(z) j_{a}\left(z^{\prime}\right) \sim \operatorname{regular},
\end{aligned}
$$

where $a, b=+,-$ or 3 . From the above OPE algebra, we learn that $J$ 's and $j$ 's together generate a superaffine algebra of $S L(2)$ at level 0 , which here, as noted in [2], appears in the Wakimoto free-field representation [32]. Indeed, these chiral vertex operators are holomorphic in $z$, which means that one can expand them in a Laurent series that allows an affinization of the $S L(2)$ superalgebra generated by their resulting zero modes. Thus, the space of global sections of $\widehat{\Omega}_{\mathbb{P}^{1}}^{\text {ch }}$ is a module for the superaffine algebra of $S L(2)$ at level 0 . The space of these operators has a structure of a chiral algebra in the full physical sense; it obeys all the physical axioms of a chiral algebra, including reparameterization invariance on the $z$-plane or worldsheet $\Sigma$. We will substantiate this last statement momentarily by showing that the holomorphic stress tensor exists in the $\bar{Q}_{+}$-cohomology.

Still on the subject of global sections, recall from Sections 6.1 and 6.2, and our $\bar{Q}_{+}$-Cech cohomology dictionary, that there will be $\psi^{\bar{i}}$-independent operators $J(z), Q(z), T(z)$, and $G(z)$ in the $\bar{Q}_{+}$-cohomology of the underlying half-twisted model on $\mathbb{C P}^{1}$ if and only if the corresponding $\widehat{J}(z), \widehat{Q}(z)$, $\widehat{T}(z)$, and $\widehat{G}(z)$ operators can be globally defined, i.e., the $\mathcal{J}(z), \mathcal{Q}(z), \mathcal{T}(z)$, and $\mathcal{G}(z)$ operators of the free $b c-\beta \gamma$ system belong in $H^{0}\left(\mathbb{C P}^{1}, \widehat{\Omega}_{\mathbb{P} 1}^{\text {ch }}\right)-$ the space of global sections of $\widehat{\Omega}_{\mathbb{P 1}}^{\mathrm{ch}}$. Let us look at this more closely.

Now, note that for $X=\mathbb{C P}^{1}$, we have

$$
\begin{aligned}
& \mathcal{J}(z)=: b c:(z) \\
& \mathcal{Q}(z)=: \beta c:(z), \\
& \mathcal{T}(z)=-: \beta \partial_{z} \gamma:(z)-: b \partial_{z} c:(z), \\
& \mathcal{G}(z)=: b \partial_{z} \gamma:(z),
\end{aligned}
$$


where the above operators are defined and regular in $U_{1}$. Similarly, we also have

$$
\begin{aligned}
& \widetilde{\mathcal{J}}(z)=: \tilde{b} \tilde{c}:(z), \\
& \widetilde{\mathcal{Q}}(z)=: \tilde{\beta} \tilde{c}:(z), \\
& \widetilde{\mathcal{T}}(z)=-: \tilde{\beta} \partial_{z} \tilde{\gamma}:(z)-: \tilde{b} \partial_{z} \tilde{c}:(z), \\
& \widetilde{\mathcal{G}}(z)=: \tilde{b} \partial_{z} \tilde{\gamma}:(z),
\end{aligned}
$$

where the above operators are defined and regular in $U_{2}$. By substituting the automorphism relations (7.5)-(7.8) into (7.27)-(7.30), a small computation shows that in $U_{1} \cap U_{2}$, we have

$$
\begin{aligned}
\widetilde{\mathcal{T}}(z) & =\mathcal{T}(z), \\
\widetilde{\mathcal{Q}}(z)-\mathcal{Q}(z) & =2 \partial_{z}\left(\frac{c}{\gamma}\right)(z), \\
\widetilde{\mathcal{G}}(z) & =\mathcal{G}(z), \\
\widetilde{\mathcal{J}}(z)-\mathcal{J}(z) & =-2\left(\frac{\partial_{z} \gamma}{\gamma}\right)(z),
\end{aligned}
$$

where an operator that is a global section of $\widehat{\Omega}_{\mathbb{P}^{1}}^{\text {ch }}$ must agree in $U_{1} \cap U_{2}$.

Notice that in $U_{1} \cap U_{2}$, we have $\widetilde{\mathcal{J}} \neq \mathcal{J}$ and $\widetilde{\mathcal{Q}} \neq \mathcal{Q}$. The only way to consistently modify $\mathcal{J}$ and $\widetilde{\mathcal{J}}$ so as to agree on $U_{1} \cap U_{2}$ is to shift them by a multiple of the term $\left(\partial_{z} \gamma\right) / \gamma=-\left(\partial_{z} \tilde{\gamma}\right) / \tilde{\gamma}$. However, this term has a pole at both $\gamma=0$ and $\tilde{\gamma}=0$. Thus, it cannot be used to redefine $\mathcal{J}$ or $\tilde{\mathcal{J}}$ (which has to be regular in $U_{1}$ or $U_{2}$, respectively). The only way to consistently modify $\mathcal{Q}$ and $\widetilde{\mathcal{Q}}$ so as to agree on $U_{1} \cap U_{2}$ is to shift them by a linear combination of the terms $\left(\partial_{z} c\right) / \gamma=-\tilde{\gamma} \partial_{z}\left(\tilde{c} / \tilde{\gamma}^{2}\right)$ and $\left(c \partial_{z} \gamma\right) / \gamma^{2}=\left(\tilde{c} \partial_{z} \tilde{\gamma}\right) / \tilde{\gamma}^{2}$. Similarly, these terms have poles at both $\gamma=0$ and $\tilde{\gamma}=0$, and hence, cannot be used to redefine $\mathcal{Q}$ or $\widetilde{\mathcal{Q}}$ (which also has to be regular in $U_{1}$ or $U_{2}$, respectively).

Therefore, we conclude that $\mathcal{T}(z)$ and $\mathcal{G}(z)$ belong in $H^{0}\left(\mathbb{C P}^{1}, \widehat{\Omega}_{\mathbb{P}^{1}}^{\text {ch }}\right)$, while $\mathcal{J}(z)$ and $Q(z)$ do not belong in $H^{0}\left(\mathbb{C P}^{1}, \widehat{\Omega}_{\mathbb{P}^{1}}^{\text {ch }}\right)$. This means that $T(z)$ and $G(z)$ are in the $\bar{Q}_{+}$-cohomology of the underlying half-twisted $(2,2)$ model on $\mathbb{C P}^{1}$, while $J(z)$ and $Q(z)$ are not. This last statement is in perfect agreement with the physical picture presented in Section 6.1, which states that since $c_{1}\left(\mathbb{C P}^{1}\right) \neq 0$, the symmetries associated with $J(z)$ and $Q(z)$ ought to be broken such that $J(z)$ and $Q(z)$ will cease to exist in the $\bar{Q}_{+}$-cohomology at the quantum level. Moreover, it is also stated in Section 6.1, that the symmetries associated with $T(z)$ and $G(z)$ are exact in quantum perturbation theory, and that these operators will remain in the $\bar{Q}_{+}$-cohomology at the quantum level. This just corresponds to the mathematical fact that the sheaf 
$\widehat{\Omega}_{X}^{\text {ch }}$ of CDR on any $X$ has the structure of conformal vertex superalgebra, such that the conformal anomaly discussed in Section 5.7 vanishes regardless of the value of $c_{1}(X)$, i.e., we have $\widetilde{\mathcal{T}}=\mathcal{T}$ and $\widetilde{\mathcal{G}}=\mathcal{G}$ always. Via (7.31)-(7.34), we obtain a purely mathematical interpretation of a physical result concerning the holomorphic structure of the underlying, "massive" half-twisted model on $\mathbb{C P}^{1}$; the reduction from an $N=2$ to an $N=1$ algebra in the holomorphic structure of the half-twisted model on $\mathbb{C P}^{1}$ (with $c_{1}\left(\mathbb{C P}^{1}\right)$ being proportional to its non-zero 1-loop beta function), is due to an obstruction in gluing $\mathcal{J}(z)$ 's and $\mathcal{Q}(z)$ 's (on overlaps) as global sections of the sheaf $\widehat{\Omega}_{\mathbb{P}^{1}}^{\text {ch }}$ of $\mathrm{CDR}$ on $\mathbb{C P}^{1}$.

As mentioned in Section 6.1, the symmetries associated with the currents $J(z)$ and $Q(z)$ will remain unbroken in the conformal limit where $c_{1}(X)=0$, i.e., if the sigma model 1-loop beta-function vanishes. Thus, one is led to the following question: is the non-vanishing of the obstruction terms on the RHS of (7.32) and (7.34) due to a non-zero 1-loop beta-function? And will they vanish if the 1-loop beta-function is zero?

In order to answer this question, first recall from the $\mathbb{C P}^{1}$ example in Section 5.7 that we have a correspondence between the holomorphic term $\left(\partial_{z} \gamma\right) / \gamma$ and the sigma model operator $R_{i \bar{j}} \partial_{z} \phi^{i} \psi^{\bar{j}}$. Hence, since $R_{i \bar{j}}$ is proportional to the 1-loop beta function, we find that the RHS of (7.34) will also be proportional to the 1-loop beta-function. Consequently, $\mathcal{J}(z)$ will be a global section of $\widehat{\Omega}_{\mathbb{P 1}}^{\text {ch }}$ and hence, $J(z)$ will be in the $\bar{Q}_{+}$-cohomology of the half-twisted model on $\mathbb{C P}^{1}$, if and only if the 1-loop beta-function vanishes.

What about $\mathcal{Q}(z)$ and $Q(z)$ ? First, the identification $\gamma^{i}=\phi^{i}$ further implies a correspondence between the term $1 / \gamma$ and the sigma model operator $R_{i \bar{j}} \psi^{\bar{j}}$. Secondly, notice that the RHS of $(7.32)$ is given by $2\left[\left(\partial_{z} c\right) / \gamma-\right.$ $\left.\left(c \partial_{z} \gamma\right) / \gamma^{2}\right]$. Thus, via the above-mentioned correspondence between the holomorphic terms and operators, the identification $c^{i}=\psi^{i}$, and the fact that $\partial_{\gamma}(1 / \gamma)=-1 / \gamma^{2}$, we find that the physical counterpart of the RHS of (7.32) will be given by the sigma model operator $2\left[R_{i \bar{j}} \partial_{z} \psi^{i} \psi^{\bar{j}}+\right.$ $\left.R_{i \bar{j}, k} \psi^{k} \partial_{z} \phi^{i} \psi^{\bar{j}}\right]$. Therefore, we see that the RHS of (7.32) is proportional to the 1-loop beta-function as well. Consequently, $\mathcal{Q}(z)$ will be a global section of $\widehat{\Omega}_{\mathbb{P}^{1}}^{\text {ch }}$ and hence, $Q(z)$ will be in the $\bar{Q}_{+}$-cohomology of the half-twisted model on $\mathbb{C P}^{1}$, if and only if the 1-loop beta-function vanishes.

Note that the above identification of the holomorphic terms with sigma model operators involving the Ricci tensor (and therefore the 1-loop beta function) and its derivatives is consistent with the relations $[\mathcal{Q}, \mathcal{J}(z)]=$ $-\mathcal{Q}(z)$ and $[\mathcal{Q}, \widetilde{\mathcal{J}}(z)]=-\widetilde{\mathcal{Q}}(z)$ of the free conformal field theories in $U_{1}$ and 
$U_{2}$, respectively. This can be seen as follows. First, notice that if $[\mathcal{Q}, \mathcal{J}(z)]=$ $-\mathcal{Q}(z)$ holds in the open set $U_{1}$, then $\widetilde{\mathcal{Q}}(z)-\mathcal{Q}(z)=[\mathcal{Q}, \mathcal{J}(z)-\widetilde{\mathcal{J}}(z)]$ must hold in the overlap $U_{1} \cap U_{2}$. And if we can represent $\widetilde{\mathcal{J}}(z)-\mathcal{J}(z)$ by the sigma model operator $-2 R_{i \bar{j}} \partial_{z} \phi^{i} \psi^{\bar{j}}$, it will mean that we can represent $\widetilde{\mathcal{Q}}(z)-\mathcal{Q}(z)$ by the sigma model operator $\left\{\mathcal{Q}, 2 R_{i \bar{j}} \partial_{z} \phi^{i} \psi^{\bar{j}}\right\}=2\left[R_{i \bar{j}} \partial_{z} \psi^{i} \psi^{\bar{j}}+\right.$ $\left.R_{i \bar{j}, k} \psi^{k} \partial_{z} \phi^{i} \psi^{\bar{j}}\right]$, where again we have made use of the identification $\gamma^{i}=\phi^{i}$ and $c^{i}=\psi^{i}$ and the field variations $\delta \gamma^{i}=c^{i}$ and $\delta b_{i}=-\beta_{i}$ generated by $\mathcal{Q}$. These expressions coincide with those in the last paragraph. Clearly, this verification lends further support to the interpretation of the 1-loop beta-function in terms of holomorphic data in this section, as well as in Section 5.7.

Alternatively, one can try to ascertain the relationship between the obstructing terms on the RHS of (7.32) and (7.34), and the first Chern class $c_{1}(X)$ for $X=\mathbb{C P}^{1}$. One can then check to see if there is any correlation between a non-vanishing obstruction and a non-zero first Chern class, and vice-versa. To this end, one may substitute the automorphism relations (6.30)-(6.33) into $\widetilde{\mathcal{J}}(z), \widetilde{\mathcal{Q}}(z), \widetilde{\mathcal{T}}(z)$ and $\widetilde{\mathcal{G}}(z)$ and compute that for any $X[1]$

$$
\begin{aligned}
\widetilde{\mathcal{T}}(z) & =\mathcal{T}(z) \\
\widetilde{\mathcal{Q}}(z)-\mathcal{Q}(z) & =\partial_{z}\left(\frac{\partial}{\partial \tilde{\gamma}^{k}}\left[\operatorname{Tr} \ln \left(\frac{\partial \gamma^{i}}{\partial \tilde{\gamma}^{j}}\right)\right] \tilde{c}^{k}(z)\right), \\
\widetilde{\mathcal{G}}(z) & =\mathcal{G}(z) \\
\widetilde{\mathcal{J}}(z)-\mathcal{J}(z) & =\partial_{z}\left(\operatorname{Tr} \ln \left(\frac{\partial \tilde{\gamma}^{i}}{\partial \gamma^{j}}\right)\right),
\end{aligned}
$$

where $i, j, k=1, \ldots, \operatorname{dim}_{\mathbb{C}} X$. It has indeed been shown in [1] that the terms on the RHS of (7.36) and (7.38) vanish if and only if $c_{1}(X)=0$, whence the structure of the sheaf $\widehat{\Omega}_{X}^{\text {ch }}$ of CDR is promoted to that of a topological vertex superalgebra, with global sections $\mathcal{T}(z), \mathcal{G}(z), \mathcal{J}(z)$, and $\mathcal{Q}(z)$ obeying the OPEs in (6.10) of a holomorphic, twisted $N=2$ superconformal algebra. In short, the terms on the RHS of (7.32) and (7.34) appear because $c_{1}\left(\mathbb{C P}^{1}\right) \neq$ 0 , where hypothetically speaking, they would have vanished if $c_{1}\left(\mathbb{C P}^{1}\right)$ had been zero.

The half-twisted $(2,2)$ model on $\mathbb{C P}^{1}$ is an example of a theory which has a holomorphic stress tensor in its chiral algebra but a non-zero 1-loop beta-function. Thus, one can expect the theory to flow to a fixed point in the infrared where its (twisted) $N=2$ superconformal invariance will be restored. 


\subsubsection{The first cohomology}

We shall now proceed to investigate the first cohomology group $H^{1}\left(\mathbb{C P}^{1}, \widehat{\Omega}_{\mathbb{P}^{1}}^{\text {ch }}\right)$.

In dimension 0 , we again have functions that are of a higher degree in $\gamma$ but of a single degree in $c$ as possible candidates. However, from ordinary algebraic geometry, we have the classical result that $H^{1}\left(\mathbb{C P}^{1}, \mathcal{O}\right)=0$, where $\mathcal{O}$ is the sheaf of functions over $\mathbb{C P}^{1}$, which are holomorphic in $\gamma$. Since a vanishing cohomology at the classical level continues to vanish at the quantum level, we learn that we cannot have functions which are monomials in $\gamma$.

That leaves us to consider polynomials of the form $f(\gamma) c$ or the monomial $c$. In order to determine if they belong in the first cohomology, let us first recall that $c$ is a local section of the pull-back of the holomorphic tangent bundle of $\mathbb{C P}^{1}$, i.e., $c \in \mathcal{O}\left(\gamma^{*} T \mathbb{C P}^{1}\right)$. Hence, polynomials of the form $f(\gamma) c$ are sections of the sheaf $\mathcal{O} \otimes \mathcal{O}\left(\gamma^{*} T \mathbb{C P}^{1}\right)$. From the cup product map, we have

$$
\begin{aligned}
& {\left[H^{0}\left(\mathbb{C P}^{1}, \mathcal{O}\right) \otimes H^{1}\left(\mathbb{C P}^{1}, \mathcal{O}\left(\gamma^{*} T \mathbb{C P}^{1}\right)\right)\right] \oplus\left[H^{1}\left(\mathbb{C P}^{1}, \mathcal{O}\right)\right.} \\
& \left.\otimes H^{0}\left(\mathbb{C P}^{1}, \mathcal{O}\left(\gamma^{*} T \mathbb{C P}^{1}\right)\right)\right] \longrightarrow H^{1}\left(\mathbb{C P}^{1}, \mathcal{O} \otimes \mathcal{O}\left(\gamma^{*} T \mathbb{C P}^{1}\right)\right)
\end{aligned}
$$

Since $H^{1}\left(\mathbb{C P}^{1}, \mathcal{O}\right)=0$, and $H^{0}\left(\mathbb{C P}^{1}, \mathcal{O}\right)$ is generated by 1 , we effectively have the map

$$
H^{1}\left(\mathbb{C P}^{1}, \mathcal{O}\left(\gamma^{*} T \mathbb{C P}^{1}\right)\right) \longrightarrow H^{1}\left(\mathbb{C P}^{1}, \mathcal{O} \otimes \mathcal{O}\left(\gamma^{*} T \mathbb{C P}^{1}\right)\right)
$$

From chiral Poincaré duality [31], we have the relation $H^{0}\left(\mathbb{C P}^{1}, \widehat{\Omega}_{\mathbb{P}^{1} ; n}^{\mathrm{ch} ; p}\right)^{*}=$ $H^{1}\left(\mathbb{C P}^{1}, \widehat{\Omega}_{\mathbb{P}^{1} ; n}^{\mathrm{ch} ; 1-p}\right)$. Thus, $H^{0}\left(\mathbb{C P}^{1}, \widehat{\Omega}_{\mathbb{P}^{1} ; 0}^{\mathrm{ch} 0}\right)^{*}=H^{1}\left(\mathbb{C P}^{1}, \widehat{\Omega}_{\mathbb{P}^{1} ; 0}^{\mathrm{ch} ; 1}\right)$, where the sections of the sheaf $\widehat{\Omega}_{\mathbb{P}^{1} ; 0}^{\text {ch;0 }}$ are given by holomorphic functions $f(\gamma)$, while the sections of the sheaf $\widehat{\Omega}_{\mathbb{P}^{1} ; 0}^{\text {ch;1 }}$ are given by polynomials $f(\gamma) c$ or just $c$. Recall that $H^{0}\left(\mathbb{C P}^{1}, \widehat{\Omega}_{\mathbb{P}^{1} ; 0}^{\mathrm{ch} ; 0}\right) \cong \mathbb{C}$ and is thus one-dimensional. Hence, we learn from chiral Poincaré duality that $H^{1}\left(\mathbb{C P}^{1}, \widehat{\Omega}_{\mathbb{P}^{1} ; 0}^{\mathrm{ch} ; 1}\right)$ must also be one-dimensional. Together with map (7.40), we find that $H^{1}\left(\mathbb{C P}^{1}, \widehat{\Omega}_{\mathbb{P}^{1} ; 0}^{\text {ch; }}\right)$, the first cohomology group at dimension 0 , must be one-dimensional and generated by $c$.

In dimension 1, we will need to consider functions which are linear in $\beta$, $b, \partial_{z} \gamma$, or $\partial_{z} c$. One clue that we have is the standard result from algebraic geometry that $H^{1}\left(\mathbb{C P}^{1}, K\right) \neq 0$, where $K$ is the sheaf of holomorphic differentials $d \gamma / \gamma$. This implies that $\partial_{z} \gamma / \gamma$ ought to generate a dimension 1 class of the cohomology group $H^{1}\left(\mathbb{C P}^{1}, \widehat{\Omega}_{\mathbb{P}^{1}}^{\text {ch }}\right)$. However, recall that we have the 
result

$$
\widetilde{\mathcal{J}}(z)-\mathcal{J}(z)=-2\left(\frac{\partial_{z} \gamma}{\gamma}\right)(z)
$$

Since $\widetilde{\mathcal{J}}$ and $\mathcal{J}$ are by definition holomorphic in $U_{2}$ and $U_{1}$, respectively, it will mean that $\partial_{z} \gamma / \gamma$ cannot be a dimension 1 element of the group $H^{1}\left(\mathbb{C P}^{1}, \widehat{\Omega}_{\mathbb{P}^{1}}^{\mathrm{ch}}\right)$. This is because it can be written as a difference between a term that is holomorphic in $U_{2}$ and a term that is holomorphic in $U_{1}$. Thus, although $H^{1}\left(\mathbb{C P}^{1}, K\right)$ is non-vanishing classically, $\partial_{z} \gamma / \gamma \notin H^{1}\left(\mathbb{C P}^{1}, \widehat{\Omega}_{\mathbb{P}^{1} ; 1}^{\text {ch;0 }}\right)$ due to quantum effects in perturbation theory.

Similarly, since we have the result

$$
\widetilde{\mathcal{Q}}(z)-\mathcal{Q}(z)=2 \partial_{z}\left(\frac{c}{\gamma}\right)(z)
$$

it will mean that $\partial_{z}(c / \gamma)$ cannot be a dimension 1 element of the first cohomology group $H^{1}\left(\mathbb{C P}^{1}, \widehat{\Omega}_{\mathbb{P}^{1}}^{\mathrm{ch}}\right)$. Specifically, $\partial_{z}(c / \gamma) \notin H^{1}\left(\mathbb{C P}^{1}, \widehat{\Omega}_{\mathbb{P}^{1} ; 1}^{\mathrm{ch} ; 1}\right)$.

Apart from the above two examples which are inadmissible (due to quantum effects) as elements of the first cohomology, we can obtain other admissible, dimension 1 operators in the first cohomology by acting on $c$ with dimension 1 global sections $\left\{J_{+}, J_{-}, J_{3}\right\}$ and $\left\{j_{+}, j_{-}, j_{3}\right\}$ of $\widehat{\Omega}_{\mathbb{P}^{1}}^{\text {ch }} \cdot{ }^{28}$ In addition, from the chiral Poincaré duality, we have the relations $H^{0}\left(\mathbb{C P}^{1}, \widehat{\Omega}_{\mathbb{P}^{1} ; 1}^{\mathrm{ch} ; 0}\right)^{*}=$ $H^{1}\left(\mathbb{C P}^{1}, \widehat{\Omega}_{\mathbb{P}^{1} ; 1}^{\text {ch; }}\right)$ and $H^{0}\left(\mathbb{C P}^{1}, \widehat{\Omega}_{\mathbb{P}^{1} ; 1}^{\mathrm{ch} ;-1}\right)^{*}=H^{1}\left(\mathbb{C P}^{1}, \widehat{\Omega}_{\mathbb{P}^{1} ; 1}^{\mathrm{ch} ; 2}\right)$, where classes in $H^{1}$ $\left(\mathbb{C P}^{1}, \widehat{\Omega}_{\mathbb{P}^{1} ; 1}^{\mathrm{ch} ; 2}\right)$ and $H^{1}\left(\mathbb{C P}^{1}, \widehat{\Omega}_{\mathbb{P}^{1} ; 1}^{\mathrm{ch} ;}\right)$ are represented by bosonic and fermionic operators, respectively. Since $\left\{J_{+}, J_{-}, J_{3}\right\} \in H^{0}\left(\mathbb{C P}^{1}, \widehat{\Omega}_{\mathbb{P}^{1} ; 1}^{\mathrm{ch} ; 0}\right)$ and $\left\{j_{+}, j_{-}, j_{3}\right\}$ $\in H^{0}\left(\mathbb{C P}^{1}, \widehat{\Omega}_{\mathbb{P}^{1} ; 1}^{\mathrm{ch} ;-1}\right)$, we find that the space $H^{1}\left(\mathbb{C P}^{1}, \widehat{\Omega}_{\mathbb{P}^{1}}^{\text {ch }}\right)$ is also a module for a superaffine algebra of $S L(2)$ at level 0 . We will omit the computation of these operators for brevity.

In dimension 2 and higher, we do not have relations that are analogous to (7.41) and (7.42) in dimension 1. Thus, we could very well borrow the results from standard algebraic geometry to ascertain the relevant operators of dimension 2 and higher in the first cohomology. We will again omit the computation of these operators for brevity.

\footnotetext{
${ }^{28}$ This approach is feasible because we have the cup product mapping of Cech cohomology groups: $H^{0}\left(\mathbb{C P}^{1}, \mathcal{B}\right) \otimes H^{1}\left(\mathbb{C P}^{1}, \mathcal{D}\right) \rightarrow H^{1}\left(\mathbb{C P}^{1}, \mathcal{K}\right)$, where $\mathcal{B} \otimes \mathcal{D} \rightarrow \mathcal{K}$, and $\mathcal{B}, \mathcal{D}$, and $\mathcal{K}$ are relevant sheaves.
} 


\subsection{The half-twisted $(2,2)$ model on $\mathrm{S}^{3} \times \mathrm{S}^{1}$}

As shown earlier in Section 3.3, the twisted version of the usual $(0,2)$ heterotic sigma model can be related to a unitary model with $(0,2)$ supersymmetry. Likewise on the $(2,2)$ locus, the half-twisted $(2,2)$ model can be related to a unitary model with $(2,2)$ supersymmetry. Hence, if we are to allow for the possibility of constructing sheaves of CDOs on the target space $X$, then $X$ should be non-Kähler with torsion, just as in the $(0,2)$ case. $^{29}$

It is commonly known that a $(2,2)$ model formulated using only chiral superfields does not admit non-Kähler target spaces [33]. However, if the model is being formulated in terms of chiral and twisted chiral superfields, one can allow for non-Kähler target spaces with torsion [34]. An example of a non-Kähler complex manifold that exists as the target space of a $(2,2)$ sigma model is $X=\mathbf{S}^{\mathbf{3}} \times \mathbf{S}^{\mathbf{1}}$. In fact, an off-shell construction of this model has been given in [35], where it is also shown that for a $(2,2)$ sigma model on a group manifold, the only example amenable to such a formulation is the parallelized group manifold $X=S U(2) \times U(1) \cong \mathbf{S}^{\mathbf{3}} \times \mathbf{S}^{\mathbf{1}}$. Moreover, a hermitian form $\omega_{T}$, which defines the torsion on $\mathbf{S}^{\mathbf{3}} \times \mathbf{S}^{\mathbf{1}}$ while obeying the weaker condition $\partial \bar{\partial} \omega_{T}=0$, has also been explicitly derived in [12]. Let us therefore explore this model further.

\subsubsection{The WZW model}

As explained in $[35,36]$, the $(2,2)$ model on $\mathbf{S}^{\mathbf{3}} \times \mathbf{S}^{\mathbf{1}}$ is a tensor product of an $S U(2)$ WZW model, times a free field theory on $\mathbf{S}^{\mathbf{1}}$, times four free left- and right-moving real fermions. The real fermions combine into four complex fermions which transform in the adjoint representation of $S U(2) \times U(1)$, i.e., $\mathbf{3}$ of $S U(2)$ and $\mathbf{1}$ of $U(1)$. The $S U(2)$ fermions are free because the connection on $S U(2)$, which follows from the $(2,2)$ model on $\mathbf{S}^{\mathbf{3}} \times \mathbf{S}^{\mathbf{1}}$, has torsion, and is parallelized. ${ }^{30}$ There is thus a shift in the level of the $S U(2)$ WZW model due to a relevant redefinition of these fermionic fields. This will be apparent shortly.

On the $(2,2)$ locus, the left- and right-moving fermions are equivalent to a set of fermionic $b c$ and $\tilde{b} \tilde{c}$ fields (labelled by $\psi_{z}^{\bar{i}}, \psi^{i}$ and $\psi_{\bar{z}}^{i}, \psi^{\bar{i}}$ in Section 6.1) with spins 1 and 0 , respectively. The $b c$ and $\tilde{b} \tilde{c}$ systems have left and right central charges $(-2,0)$ and $(0,-2)$. On a manifold such as $\mathbf{S}^{\mathbf{3}} \times \mathbf{S}^{\mathbf{1}}$

\footnotetext{
${ }^{29}$ Recall from the discussion in Section 3.3 on the relation with unitary models with $(0,2)$ supersymmetry that the non-Kählerity and torsion of the target space are required to define the moduli of the sheaves of CDOs.

${ }^{30}$ The author would like to thank M. Rocek for helpful Email correspondences on this particular point.
} 
with complex dimension 2 , there will be two sets of left- and right-moving fermions. Hence, the fermions contribute a total of $(-4,-4)$ to the left and right central charges of the model. The $S U(2)$ WZW model at level $k$ contributes $(3 k /(k+2), 3 k /(k+2))$ to the central charges, and the free theory on $\mathbf{S}^{1}$ contributes $(1,1)$. The total left and right central charges are therefore $(3 k /(k+2)-3,3 k /(k+2)-3)$. The difference between the left and right central charges remains the same in passing from the physical theory to the $\bar{Q}_{+}$-cohomology. In this example, it is given by $c=0$. This should be the central charge of the stress tensor which will appear as a global section of the sheaf of CDOs, which in this case, is the sheaf $\widehat{\Omega}_{X}^{\text {ch }}$ of CDR on $X=\mathbf{S}^{\mathbf{3}} \times \mathbf{S}^{\mathbf{1}}$.

Similarly, we can pre-ascertain the central charges of the current algebra which will be furnished by the appropriate global sections of the sheaf of CDR on $\mathbf{S}^{\mathbf{3}} \times \mathbf{S}^{\mathbf{1}}$. The underlying $S U(2)$ WZW model has an $S U(2)$-valued field $g$, with symmetry $S U(2)_{\mathrm{L}} \times S U(2)_{\mathrm{R}}$ (to be precise, it is $\left(S U(2)_{\mathrm{L}} \times\right.$ $\left.S U(2)_{\mathrm{R}}\right) / \mathbb{Z}_{2}$, where $\mathbb{Z}_{2}$ is the common centre of the two factors). The symmetry acts by $g \rightarrow a g b^{-1}, a, b \in S U(2)$. In the WZW model, the $S U(2)_{\mathrm{L}}$ symmetry is part of a holomorphic $S U(2)$ current algebra of level $k+2$, while $S U(2)_{\mathrm{R}}$ is part of an anti-holomorphic $S U(2)$ current algebra of level $k+2$. As mentioned earlier, the shift by " 2 " in the level of the $S U(2)$ current algebra is expected, and it is due to the fact that the complex fermions transform freely in the adjoint representation of $S U(2)$. The left and right central charges are therefore $(k+2,0)$ for $S U(2)_{\mathrm{L}}$ and $(0, k+2)$ for $S U(2)_{\mathrm{R}}$.

Next, notice that the (right-moving) supersymmetry generator $\bar{Q}_{+}$, although invariant under a left-moving $U(1)$ current, is nevertheless charged under a right-moving one. (Recall from Section 2.2 that $\bar{Q}_{+}$has charge $\left(q_{\mathrm{L}}, q_{\mathrm{R}}\right)=(0,+1)$.) Hence, the physical characteristics of $\bar{Q}_{+}$, and the symmetry of the $\bar{Q}_{+}$-cohomology that it defines, will depend on the twist one makes on the right-moving fields. Since the twisting of the four real rightmoving fermions of the underlying $(2,2)$ model on $\mathbf{S}^{\mathbf{3}} \times \mathbf{S}^{\mathbf{1}}$ reduces the $S U(2)_{\mathrm{R}}$ symmetry to its maximal torus $U(1)_{\mathrm{R}}$, the symmetry that should survive at the level of the $\bar{Q}_{+}$-cohomology or sheaf of CDR is $\left(S U(2)_{\mathrm{L}} \times\right.$ $\left.U(1)_{\mathrm{R}}\right) / \mathbb{Z}_{2}=U(2)$.

The difference between the left and right central charges remains the same in passing to the $\bar{Q}_{+}$-cohomology or sheaf of CDR. Hence, the expected levels of the $S U(2)_{\mathrm{L}}$ and $U(1)_{\mathrm{R}}$ current algebras, furnished by global sections of the sheaf of CDR, should be given by $k+2$ and $-k-2$, respectively. The only case in which they are equal is $k=-2$, for which the levels are both 0 . This is not really a physically sensible value for the WZW model; physically sensible, unitary WZW models with convergent path integrals must be restricted to integer values of $k$ with $k \geq 0$. However, as we will 
see shortly, $k$ is, in our case at hand, an arbitrary complex parameter that is directly related to the moduli of the sheaves of CDR (that are in turn represented by $\left.H^{1}\left(\mathbf{S}^{\mathbf{3}} \times \mathbf{S}^{\mathbf{1}}, \Omega^{2, c l}\right) \cong \mathbb{C}\right)$.

In the sheaf of CDR, the symmetries are readily complexified, so that the symmetry of the corresponding current algebra which appears should be at the Lie algebra level $G L(2)$ instead of $U(2)$. Likewise, with respect to the $S U(2)$ and $U(1)$ subgroups of $G L(2)$, the symmetry of the corresponding current algebra that will appear should be given by $S L(2)$ and $G L(1)$, respectively. In addition, the $U(1)_{\mathrm{R}}$ (which acts on the coordinate variables $v^{i}$, to be introduced shortly, by $\left.v^{i} \rightarrow \mathrm{e}^{i \theta} v^{i}\right)$ and the rotation of $\mathbf{S}^{1}$ (which acts by $v^{i} \rightarrow \mathrm{e}^{\chi} v^{i}$ with real $\chi$ ) combine together to generate the centre of $G L(2)$. At the Lie algebra level, the centre is $G L(1)$. This is the symmetry that we will expect to see as well. Note that the rotation of $\mathbf{S}^{1}$ will always correspond to a $U(1)$ current algebra with equal left and right central charges. Thus, it will not affect our above discussion whereby only the differences between the left and right central charges are important.

\subsubsection{Constructing a sheaf of CDR on $\mathrm{S}^{3} \times \mathrm{S}^{1}$}

We now proceed towards our main objective of constructing a family of sheaves of CDR on $\mathbf{S}^{\mathbf{3}} \times \mathbf{S}^{\mathbf{1}}$. As a starter, we will first construct a sheaf of CDR without introducing any modulus. At this point, one would already be able to see, within the current algebras derived, the expected symmetries discussed above. Thereafter, we will generalize the construction and introduce a variable parameter which will serve as the modulus of the sheaves of CDR. It is at this juncture that we find an explicit relation between the modulus of the sheaves and the level of the underlying $S U(2)$ WZW model.

Let us begin by noting that $\mathbf{S}^{\mathbf{3}} \times \mathbf{S}^{\mathbf{1}}$ can be expressed as $\left(\mathbb{C}^{2}-\{0\}\right) / \mathbb{Z}$, where $\mathbb{C}^{2}$ has coordinates $v^{1}, v^{2}$, and $\{0\}$ is the origin in $\mathbb{C}^{2}$ (the point $v^{1}=v^{2}=0$ ) which should be removed before dividing by $\mathbb{Z}$. Also, $\mathbb{Z}$ acts by $v^{i} \rightarrow \lambda^{n} v^{i}$, where $\lambda$ is a non-zero complex number of modulus less than 1 and $n$ is any integer. $\lambda$ is a modulus of $\mathbf{S}^{\mathbf{3}} \times \mathbf{S}^{\mathbf{1}}$ that we shall keep fixed.

To construct the most basic sheaf of CDR with target $\mathbf{S}^{\mathbf{3}} \times \mathbf{S}^{\mathbf{1}}$, one simply defines the scalar coordinate variables $v^{i}$ as free bosonic fields of spin 0 , with conjugate spin 1 fields $V_{i}$. From our earlier discussions, one will also need to introduce fermionic fields $w^{i}$ of spin 0 , with conjugate spin 1 fields $W_{i}$. Since $\mathbf{S}^{\mathbf{3}} \times \mathbf{S}^{\mathbf{1}}$ has complex dimension 2, the index $i$ in all fields will run from 1 to 2 . Therefore, the free field action that one must consider is given by

$$
I=\frac{1}{2 \pi} \int\left|d^{2} z\right|\left(V_{1} \bar{\partial} v^{1}+V_{2} \bar{\partial} v^{2}+W_{1} \bar{\partial} w^{1}+W_{2} \bar{\partial} w^{2}\right) .
$$


Notice that the above $V v-W w$ system is just the usual $\beta \gamma-b c$ system with non-trivial OPEs $V_{i}(z) v^{j}\left(z^{\prime}\right) \sim-\delta_{j}^{i} /\left(z-z^{\prime}\right)$ and $W_{i}(z) w^{j}\left(z^{\prime}\right) \sim \delta_{j}^{i} /\left(z-z^{\prime}\right)$.

In the above representation of $\mathbf{S}^{\mathbf{3}} \times \mathbf{S}^{\mathbf{1}}$, the action of $\mathbb{Z}$ represents a geometrical symmetry of the system. Thus, the only allowable operators are those which are invariant under the finite action of $\mathbb{Z}$. These operators will therefore span the space of global sections of the sheaf of CDR. Under this symmetry, $v^{i}$ transforms as $v^{i} \rightarrow \tilde{v}^{i}=\lambda v^{i}$. In order to ascertain how the rest of the fields ought to transform under this symmetry, we simply substitute $v^{i}$ and $\tilde{v}^{i}$ (noting that it is equivalent to $\gamma^{i}$ and $\tilde{\gamma}^{i}$, respectively) into (6.30)-(6.33). In short, the only allowable operators are those which are invariant under $v^{i} \rightarrow \lambda v^{i}, V_{i} \rightarrow \lambda^{-1} V_{i}, w^{i} \rightarrow \lambda w^{i}$, and $W^{i} \rightarrow \lambda^{-1} W^{i}$.

One operator that possesses this invariance is the stress tensor

$$
T_{z z}=\frac{1}{2 \pi} \sum_{i}\left(V_{i} \partial v^{i}+W_{i} \partial w^{i}\right) .
$$

Similar to the $\mathbb{C P}^{1}$ example, a stress tensor exists in the chiral algebra of the model on $\mathbf{S}^{\mathbf{3}} \times \mathbf{S}^{\mathbf{1}}$. This should come as no surprise; as explained previously, one can always find, in the CDR case, a global definition for the stress tensor, which in turn ensures its existence on any manifold. Note also that since $\mathbf{S}^{\mathbf{3}} \times \mathbf{S}^{\mathbf{1}}$ is parallelized, its first Chern class vanishes. Hence, the chiral algebra of the described theory is conformally invariant, and the sheaf of CDR has a structure of a topological vertex (super)algebra defined in [1]. This reflects the superconformal invariance of the underlying $(2,2)$ model on $\mathbf{S}^{\mathbf{3}} \times \mathbf{S}^{\mathbf{1}}$. A bosonic $\beta \gamma$ system of spins 1 and 0 has central charge $c=2$, while a fermionic $b c$ system of spins 1 and 0 has $c=-2$. Thus, the stress tensor $T$ has $c=0$, in agreement with what we had anticipated from the underlying WZW model.

The chiral algebra of the underlying model also contains dimension 1 currents $J_{j}^{i}=-\left(V_{j} v^{i}+W_{j} w^{i}\right)$. As required, these (bosonic) current operators are invariant under the field transformations $v^{i} \rightarrow \lambda v^{i}, V_{i} \rightarrow \lambda^{-1} V_{i}$, $w^{i} \rightarrow \lambda w^{i}$, and $W^{i} \rightarrow \lambda^{-1} W^{i}$. They obey the OPEs

$$
J_{j}^{i}(z) J_{l}^{m}\left(z^{\prime}\right) \sim \frac{\delta_{j}^{m} J_{l}^{i}-\delta_{l}^{i} J_{j}^{m}}{z-z^{\prime}}
$$

We recognize this as a $G L(2)$ current algebra at level 0 .

When we proceed to generalize the above construction by introducing a variable parameter to serve as the modulus of the sheaves of CDR, it will not be possible to maintain manifest $G L(2)$ symmetry. Hence, it will be useful to pick a basis in the current algebra now. The $S L(2)$ subgroup is generated 
by $J_{3}=-\frac{1}{2}\left(V_{1} v^{1}+W_{1} w^{1}-V_{2} v^{2}-W_{2} w^{2}\right), \quad J_{+}=-\left(V_{2} v^{1}+W_{2} w^{1}\right), \quad J_{-}=$ $-\left(V_{1} v^{2}+W_{1} w^{2}\right)$, with non-trivial OPEs

$$
\begin{aligned}
J_{3}(z) J_{3}\left(z^{\prime}\right) & \sim \text { reg. } \\
J_{3}(z) J_{ \pm}\left(z^{\prime}\right) & \sim \pm \frac{J_{ \pm}\left(z^{\prime}\right)}{z-z^{\prime}}, \\
J_{+}(z) J_{-}\left(z^{\prime}\right) & \sim \frac{2 J_{3}\left(z^{\prime}\right)}{z-z^{\prime}} .
\end{aligned}
$$

Notice that this is just an $S L(2)$ current algebra at level 0 . The centre of $G L(2)$ (at the Lie algebra level) is given by $G L(1)$. The corresponding current algebra is generated by $K=-\frac{1}{2}\left(V_{1} v^{1}+W_{1} w^{1}+V_{2} v^{2}+W_{1} w^{2}\right)$, with OPE given by

$$
K(z) K\left(z^{\prime}\right) \sim \operatorname{reg}
$$

This is just a $G L(1)$ current algebra at level 0 .

\subsubsection{The Modulus of CDR}

Let us now generalize the above construction of the sheaf of CDR on $\mathbf{S}^{\mathbf{3}} \times \mathbf{S}^{\mathbf{1}}$. In order to do so, we must invoke a modulus that will enable us to obtain a family of sheaves of CDR. Recall that the modulus is represented by the Cech cohomology group $H^{1}\left(\mathbf{S}^{\mathbf{3}} \times \mathbf{S}^{\mathbf{1}}, \Omega^{2, \mathrm{cl}}\right) \cong \mathbb{C}$. ${ }^{31}$ To model the modulus, one simply needs to introduce a variable complex parameter associated with $H^{1}\left(\mathbf{S}^{\mathbf{3}} \times \mathbf{S}^{\mathbf{1}}, \Omega^{2, \mathrm{cl}}\right)$.

Before we proceed any further, it will first be necessary for us to know how the relevant fields will transform under a variation of the modulus. Recall from our discussion on local symmetries in Section 5.5 that $\Omega^{2, \mathrm{cl}}$, the sheaf of closed, holomorphic $(2,0)$-forms on a manifold $X$, is associated with a non-geometrical symmetry of the free $\beta \gamma-b c$ system on $X$. Consider a general system of $n$ conjugate $\beta \gamma$ and $b c$ systems, with non-trivial OPEs $\beta_{i}(z) \gamma^{j}\left(z^{\prime}\right) \sim-\delta_{i}^{j} /\left(z-z^{\prime}\right)$ and $b_{i}(z) c^{j}\left(z^{\prime}\right) \sim \delta_{i}^{j} /\left(z-z^{\prime}\right)$, respectively. Let $F=\frac{1}{2} f_{i j}(\gamma) d \gamma^{i} \wedge d \gamma^{j}$ be a closed holomorphic two-form. Under the symmetry associated with $F$, the fields transform as

$$
\begin{aligned}
& \gamma^{j} \longrightarrow \gamma^{j}, \\
& \beta_{i} \longrightarrow \beta_{i}^{\prime}=\beta_{i}+f_{i j} \partial \gamma^{j}, \\
& c^{j} \longrightarrow c^{j}, \\
& b^{i} \longrightarrow b^{i} .
\end{aligned}
$$

\footnotetext{
${ }^{31}$ Since we are computing the short distance OPE of fields in the present context, it suffices to work locally on $\Sigma$. Hence, the modulus will be represented by $H^{1}\left(X, \Omega_{X}^{2, \mathrm{cl}}\right)$ (where $\left.X=\mathbf{S}^{\mathbf{3}} \times \mathbf{S}^{\mathbf{1}}\right)$ instead of $H^{1}\left(X \times \Sigma, \Omega_{X \times \Sigma}^{2, \text { cl }}\right)$ as stated at the end of Section 5.6.
} 
In the spirit of Section 5.5, one can verify the above transformations by locally constructing a holomorphic one-form $A=A_{i}(\gamma) d \gamma^{i}$, with $d A=F$ so that $F$ is closed, and then computing the relevant OPEs which determine how the fields transform under the action of the conserved charge $\oint A_{i} \partial_{z} \gamma^{i} d z$.

To apply the above discussion to the present case where $X=\mathbf{S}^{\mathbf{3}} \times \mathbf{S}^{\mathbf{1}}$, let us follow a strategy used in [12]. We first make a cover of $\mathbf{S}^{\mathbf{3}} \times \mathbf{S}^{\mathbf{1}}$ by two open sets $U_{1}$ and $U_{2}$, where $U_{1}$ is characterized by the condition $v^{1} \neq 0$ and $U_{2}$ by $v^{2} \neq 0$. As mentioned in [12], this is not a "good cover," as $U_{1}$ and $U_{2}$ are topologically complicated (each being isomorphic to $\mathbb{C} \times E$, where $E$ is an elliptic curve). As such, one cannot, in general, be guaranteed that an arbitrary cohomology class can be represented by a Cech cocycle with respect to this cover. However, in the present context, we have on $U_{1} \cap U_{2}$, a holomorphic section of $\Omega^{2, \mathrm{cl}}$ given by

$$
F=\frac{d v^{1} \wedge d v^{2}}{v^{1} v^{2}}
$$

Since $F$ cannot be "split" as a difference of a form holomorphic in $U_{1}$ and the one holomorphic in $U_{2}$, it thus represents an element of $H^{1}\left(\mathbf{S}^{\mathbf{3}} \times \mathbf{S}^{\mathbf{1}}, \Omega^{2, \mathrm{cl}}\right)$. From the correspondence between the $V v-W w$ and $\beta \gamma$-bc systems, the relevant field transformations are thus given by

$$
\begin{aligned}
& v^{1} \longrightarrow v^{1}, \\
& v^{2} \longrightarrow v^{2}, \\
& V_{1} \longrightarrow V_{1}^{\prime}=V_{1}+t \frac{\partial v^{2}}{v^{1} v^{2}}, \\
& V_{2} \longrightarrow V_{2}^{\prime}=V_{2}-t \frac{\partial v^{1}}{v^{1} v^{2}}, \\
& b^{1} \longrightarrow b^{1}, \\
& b^{2} \longrightarrow b^{2}, \\
& c^{1} \longrightarrow c^{1}, \\
& c^{2} \longrightarrow c^{2} .
\end{aligned}
$$

Here $t$ is a complex modulus parameter. We will see shortly that it is related to the level $k$ of the underlying $S U(2)$ WZW model. Hence, we obtain a family of sheaves of CDR, parameterized by $t$, by declaring that the fields undergo this transformation from $U_{1}$ to $U_{2}$ when we glue the sheaves together.

Let us determine how some important operators behave under this deformation. Notice that the stress tensor $T=V_{1} \partial v^{1}+V_{2} \partial v^{2}+W_{1} \partial w^{1}+W_{2} \partial w^{2}$ is invariant. Hence, the deformed theory, for any value of $t$, has a stress 
tensor of $c=0$. This is in accordance with the fact that the $(2,2)$ model on $\mathbf{S}^{\mathbf{3}} \times \mathbf{S}^{\mathbf{1}}$ is conformally invariant for all $k$ and that the difference between its left and right central charges is always 0 .

Let us now consider the $G L(1)$ current, which at $t=0$ (i.e., without considering the modulus) was defined to be $K=-\frac{1}{2}\left(V_{1} v^{1}+W_{1} w^{1}+V_{2} v^{2}+\right.$ $\left.W_{2} w^{2}\right)$. Under (7.50), we have

$$
K \longrightarrow K-\frac{t}{2}\left(\frac{\partial v_{2}}{v_{2}}-\frac{\partial v_{1}}{v_{1}}\right)=\widetilde{K}
$$

Note that the shift in $K$ under this transformation to $\widetilde{K}$ (in going from $U_{1}$ to $\left.U_{2}\right)$ is not an anomaly that spoils the existence of $K$ at $t \neq 0$. The reason is because in contrast to the situation encountered with the $\mathcal{J}(z)$ and $\mathcal{Q}(z)$ operators in the $\mathbb{C P}^{1}$ example, $\widetilde{K}-K$ can be expressed as a difference between a term (namely $t \partial v^{1} / 2 v^{1}$ ) that is holomorphic in $U_{1}$ and a term (namely $t \partial v^{2} / 2 v^{2}$ ) that is holomorphic in $U_{2}$.

Since we want to study how the current algebra will depend on $t$, it will be necessary for us to re-express the above globally defined $G L(1)$ current generator $K$ in such a way that its explicit $t$ dependence is made manifest on both $U_{1}$ and $U_{2}$. In order to be consistent with (7.51), we just need to ensure that the difference in the new expressions of $K$ in $U_{2}$ and $U_{1}$ is given by

$$
-\frac{t}{2}\left(\frac{\partial v_{2}}{v_{2}}-\frac{\partial v_{1}}{v_{1}}\right)
$$

In addition, these expressions in $U_{1}$ and $U_{2}$ must be invariant under the geometrical symmetry $v^{i} \rightarrow \lambda v^{i}$ if $K$ is to be an allowable operator. Noting these requirements, we arrive at the following; in $U_{1}$, the current is represented by

$$
K^{[1]}=-\frac{1}{2}\left(V_{1} v^{1}+W_{1} w^{1}+V_{2} v^{2}+W_{2} w^{2}\right)-\frac{t}{2} \frac{\partial v^{1}}{v^{1}},
$$

while in $U_{2}$, it is represented by

$$
K^{[2]}=-\frac{1}{2}\left(V_{1} v^{1}+W_{1} w^{1}+V_{2} v^{2}+W_{2} w^{2}\right)-\frac{t}{2} \frac{\partial v^{2}}{v^{2}} .
$$

Recalling that the original expression of $K$ given by $-\frac{1}{2}\left(V_{1} v^{1}+W_{1} w^{1}+\right.$ $\left.V_{2} v^{2}+W_{2} w^{2}\right)$ is a global section of the sheaf of CDR and is thus holomorphic in both $U_{1}$ and $U_{2}$, we see that $K^{[1]}$ and $K^{[2]}$ are holomorphic in $U_{1}$ and $U_{2}$, respectively. Moreover, as required, $K^{[1]}$ also transforms under (7.50) into $K^{[2]}$. Hence, for any value of $t$, the sheaf of CDR has a global section $K$ that is represented in $U_{1}$ by $K^{[1]}$ and in $U_{2}$ by $K^{[2]}$. 
Now we can compute the OPE singularity of $K$ for any $t$ :

$$
K(z) K\left(z^{\prime}\right) \sim-\frac{t}{2} \frac{1}{\left(z-z^{\prime}\right)^{2}} .
$$

To arrive at this result, we can either work in $U_{1}$, setting $K=K^{[1]}$ and computing the OPE, or we can work in $U_{2}$, setting $K=K^{[2]}$ and computing the OPE. The answer will come out the same in either case, because the transformation (7.50) is an automorphism of the CFT. Thus, the level of the $G L(1)$ current algebra is $-t$.

Likewise, we can work out the transformation of the $S L(2)$ currents under (7.50). The currents as defined at $t=0$, namely $J_{3}=-\frac{1}{2}\left(V_{1} v^{1}+W_{1} w^{1}-\right.$ $\left.V_{2} v^{2}-W_{2} w^{2}\right), J_{+}=-\left(V_{2} v^{1}+W_{2} w^{1}\right), J_{-}=-\left(V_{1} v^{2}+W_{1} w^{2}\right)$, transform as

$$
\begin{aligned}
& J_{3} \longrightarrow J_{3}-\frac{t}{2}\left(\frac{\partial v^{1}}{v^{1}}+\frac{\partial v^{2}}{v^{2}}\right), \\
& J_{+} \longrightarrow J_{+}+t \frac{\partial v^{1}}{v^{2}}, \\
& J_{-} \longrightarrow J_{-}-t \frac{\partial v^{2}}{v^{1}} .
\end{aligned}
$$

Similarly, the shifts in each current can be "split" as a difference of terms holomorphic in $U_{1}$ and $U_{2}$. So the currents can be re-expressed to inherit $t$-dependent terms such that they can be defined at $t \neq 0$. The new expressions of these currents, which satisfy all the necessary requirements, are given in $U_{1}$ and $U_{2}$, respectively, by

$$
\begin{aligned}
& J_{3}^{[1]}=-\frac{1}{2}\left(V_{1} v^{1}+W_{1} w^{1}-V_{2} v^{2}-W_{2} w^{2}\right)+\frac{t \partial v^{1}}{2 v^{1}}, \\
& J_{3}^{[2]}=-\frac{1}{2}\left(V_{1} v^{1}+W_{1} w^{1}-V_{2} v^{2}-W_{2} w^{2}\right)-\frac{t \partial v^{2}}{2 v^{1}},
\end{aligned}
$$

together with

$$
\begin{aligned}
& J_{+}^{[1]}=-\left(V_{2} v^{1}+W_{2} w^{1}\right) \\
& J_{+}^{[2]}=-\left(V_{2} v^{1}+W_{2} w^{1}\right)+\frac{t \partial v^{1}}{v^{2}}
\end{aligned}
$$

and

$$
\begin{aligned}
& J_{-}^{[1]}=-\left(V_{1} v^{2}+W_{1} w^{2}\right)+\frac{t \partial v^{2}}{v^{1}}, \\
& J_{-}^{[2]}=-\left(V_{1} v^{2}+W_{1} w^{2}\right) .
\end{aligned}
$$

As required, $J_{3}^{[1]}, J_{+}^{[1]}$, and $J_{-}^{[1]}$ transform into $J_{3}^{[2]}, J_{+}^{[2]}$, and $J_{-}^{[2]}$, respectively, under (7.50). Hence, for any value of $t$, the sheaf of CDR also has global 
sections $J_{3}, J_{+}$, and $J_{-}$, which are represented in $U_{1}$ by $J_{3}^{[1]}, J_{+}^{[1]}$, and $J_{-}^{[1]}$ and in $U_{2}$ by $J_{3}^{[2]}, J_{+}^{[2]}$, and $J_{-}^{[2]}$, respectively.

We shall now compute the OPEs of these current operators, working in either $U_{1}$ or $U_{2}$, whichever proves to be more convenient. We obtain an $S L(2)$ current algebra at level $t$ :

$$
\begin{aligned}
J_{3}(z) J_{3}\left(z^{\prime}\right) & \sim \frac{t}{2} \frac{1}{\left(z-z^{\prime}\right)^{2}}, \\
J_{3}(z) J_{ \pm}\left(z^{\prime}\right) & \sim \pm \frac{J_{ \pm}\left(z^{\prime}\right)}{z-z^{\prime}}, \\
J_{+}(z) J_{-}\left(z^{\prime}\right) & \sim \frac{t}{2} \frac{1}{\left(z-z^{\prime}\right)^{2}}+\frac{2 J_{3}\left(z^{\prime}\right)}{z-z^{\prime}} .
\end{aligned}
$$

The $S L(2)$ and $G L(1)$ current algebras thus have levels $t$ and $-t$, in agreement with what we had anticipated from the half-twisted $(2,2)$ model if the level $k$ of the underlying $S U(2)$ WZW theory is related to the CDR parameter $t$ by $k=t-2$; indeed at $t=0$, the levels of the $S L(2)$ and $G L(1)$ current algebras are the same at 0 , where $k=-2$.

Note that the $\bar{Q}_{+}$-cohomology of $\mathbf{S}^{\mathbf{3}} \times \mathbf{S}^{\mathbf{1}}$ does not receive instanton corrections. For any target space $X$, such corrections (because they are local on the Riemann surface $\Sigma$, albeit global in $X$ ) come only from holomorphic curves in $X$ of genus zero. There is no such curve in $\mathbf{S}^{\mathbf{3}} \times \mathbf{S}^{\mathbf{1}}{ }^{32}$ Therefore, the above analysis of the $\bar{Q}_{+}$-cohomology of $\mathbf{S}^{\mathbf{3}} \times \mathbf{S}^{\mathbf{1}}$ is exact in the full theory.

\section{Acknowledgments}

I would like to take this opportunity to thank A. Kapustin, B. Lian, M. Rocek, E. Sharpe and especially F. Malikov and E. Witten for their patience and time in educating me on various issues over our often tedious Email correspondences. I would also like to thank B. Baaquie for useful discussions.

\section{References}

[1] F. Malikov, V. Schechtman, and A. Vaintrob, Chiral De Rham complex, arXiv:math.AG/9803041.

\footnotetext{
${ }^{32}$ The author would like to thank Ed Witten for a detailed explanation of this point.
} 
[2] F. Malikov and V. Schechtman, Chiral de Rham complex, II, arXiv:math.AG/9901065.

[3] V. Gorbounov, F. Malikov and V. Schechtman, Gerbes of chiral differential operators, arXiv:math.AG/9906117.

[4] V. Gorbounov, F. Malikov and V. Schechtman, Gerbes of chiral differential operators, II, arXiv:math.AG/0003170.

[5] V. Gorbounov, F. Malikov and V. Schechtman, Gerbes of chiral differential operators, III, arXiv:math.AG/0005201.

[6] A. Beilinson and V. Drinfeld, Chiral algebras, American Mathematical Society, 2004.

[7] L. Borisov, Vertex algebras and mirror symmetry, Comm. Math. Phys. 215 (2001), 517-557.

[8] L. Borisov and A. Libgober, Elliptic genera and applications to mirror symmetry, Math.AG/9904126.

[9] L. Borisov and A. Libgober, Elliptic genera of singular varieties, preprint, arXiv:math.AG/0007108.

[10] L. Borisov and A. Libgober, Elliptic genera of singular varieties, orbifold elliptic genus and chiral de Rham complex, preprint, arXiv:math.AG/0007126.

[11] A. Kapustin, Chiral de Rham complex and the half-twisted sigma-model, arxiv:hep-th/0504074.

[12] Ed Witten, Two-Dimensional models with (0,2) supersymmetry: perturbative aspects, arXiv:hep-th/0504078.

[13] E. Frenkel and A. Losev, Mirror symmetry in two steps: A-I-B, Commun. Math. Phys. 269 (2007), 39-86, arXiv:hep-th/0505131.

[14] E. Silverstein and E. Witten, Criteria for conformal invariance of $(0,2)$ models, Nucl. Phys. B 444 (1995), 161-190, ArXiv:hep-th/9503212.

[15] S. Katz and E. Sharpe, Notes on certain (0,2) correlation functions, arXiv:hep-th/0406226.

[16] A. Adams, J. Distler and M. Ernebjerg, Topological heterotic rings, arXiv:hep-th/0506263.

[17] E. Witten, Phases of $N=2$ theories in two dimensions, Nucl. Phys. B 403 (1993), 159-222, arXiv:hep-th/9301042.

[18] M. Green, J.H. Schwarz and E. Witten, Superstring theory, Vol. II, Cambridge, Cambridge University Press, 1987.

[19] C. Hull and E. Witten, Supersymetric sigma models and the heterotic string, Phys. Lett. B 160 (1985), 398.

[20] M. Dine and N. Seiberg, (2,0) Superspace, Phys. Lett. B 180 (1986), 364. 
[21] G.W. Moore and P. Nelson, The etiology of sigma model anomalies, Commun. Math. Phys. 100 (1985), 83.

[22] R. Bott and L. Tu, Differential forms in algebraic topology, Springer, Berlin, Heidelberg, New York, 1982.

[23] M.F. Atiyah and I.M. Singer, Dirac operators coupled to vector potentials, Proc. Nat. Acad. Sci. 81 (1984), 2597.

[24] J.M. Bismut and D. Freed, The analysis of elliptic families I: metrics and connections on determinant bundles, Commun. Math. Phys. 106 (1986), 59.

[25] R.O. Wells, Differential analysis on complex manifolds, SpringerVerlag, New York, 1980.

[26] J. Distler and B. Greene, Aspects of (2,0) string compactifications, Nucl. Phys. B 304 (1988), 1-62.

[27] E. Witten, Mirror manifolds and topological field theory, in 'Essays On Mirror Manifolds', ed. S.-T. Yau, International Press, 1992, arXiv:hepth/9112056.

[28] R. Dijkgraaf, Topological field theory and 2D quantum gravity, in 'TwoDimensional Quantum Gravity and Random Surfaces', ed. D. Gross et al., World Scientific Press, Singapore, 1992, p. 191.

[29] P.S. Landweber (ed.), Elliptic curves and modular forms in algebraic topology, Springer-Verlag, 1988.

[30] R. Dijkgraaf, D. Moore, E. Verlinde and H. Verlinde, Elliptic genera of symmetric products and second quantized strings, Comm. Math. Phys. 185 (1997), 197-209.

[31] F. Malikov and V. Schechtman, Chiral poincaré duality, Math. Res. Lett. 6 (1999), 533-546.

[32] M. Wakimoto, Fock space representations of the affine lie algebra $A_{1}^{(1)}$, Commun. Math. Phys. 104 (1986), 609.

[33] P. West, Introduction to supersymmetry and supergravity (extended second version), World Scientific Press.

[34] S.J. Gates, C.M. Hull and M. Rocek, Twisted multiplets and new supersymmetric non-linear sigma models, Nucl. Phys. B 248 (1984), 157-186.

[35] M. Rocek, K. Schoutens and A. Sevrin, Off-shell WZW models in extended superspace, Phys. Lett. B 265 (1991), 303-306.

[36] Ph. Spindel, A. Sevrin, W. Troost and A. Van Proeyen, Complex structures on parallelized group manifolds and supersymmetric sigma models, Phys. Lett. B 206 (1988), 71; Extended supersymmetric sigma models on group manifolds, I. The complex structures, Nucl. Phys. B 308 (1988), 662 . 